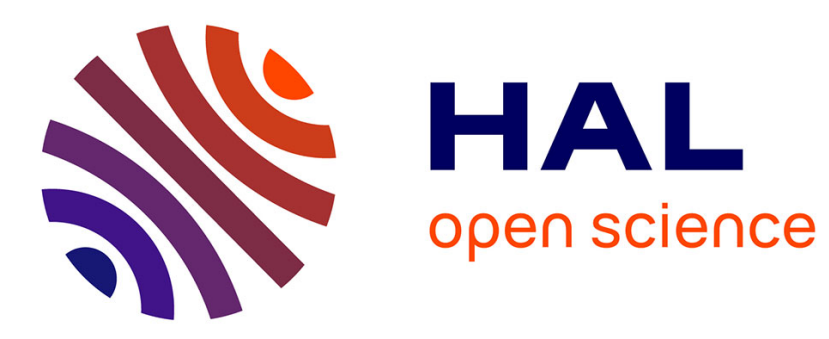

\title{
An explicit dissipative model for isotropic hard magnetorheological elastomers
}

\author{
Dipayan Mukherjee, Matthias Rambausek, Kostas Danas
}

\section{To cite this version:}

Dipayan Mukherjee, Matthias Rambausek, Kostas Danas. An explicit dissipative model for isotropic hard magnetorheological elastomers. Journal of the Mechanics and Physics of Solids, 2021, 151, pp.104361. 10.1016/j.jmps.2021.104361 . hal-03166567

\section{HAL Id: hal-03166567 https://hal.science/hal-03166567}

Submitted on 11 Mar 2021

HAL is a multi-disciplinary open access archive for the deposit and dissemination of scientific research documents, whether they are published or not. The documents may come from teaching and research institutions in France or abroad, or from public or private research centers.
L'archive ouverte pluridisciplinaire HAL, est destinée au dépôt et à la diffusion de documents scientifiques de niveau recherche, publiés ou non, émanant des établissements d'enseignement et de recherche français ou étrangers, des laboratoires publics ou privés. 


\title{
An explicit dissipative model for isotropic hard magnetorheological elastomers
}

\author{
Dipayan Mukherjee, Matthias Rambausek, Kostas Danas* \\ LMS, C.N.R.S, École Polytechnique, Institut Polytechnique de Paris, Palaiseau, 91128, France
}

\begin{abstract}
Hard magnetorheological elastomers ( $h$-MREs) are essentially two phase composites comprising permanently magnetizable metallic inclusions suspended in a soft elastomeric matrix. This work provides a thermodynamically consistent, microstructurally-guided modeling framework for isotropic, incompressible $h$-MREs. Energy dissipates in such hardmagnetic composites primarily via ferromagnetic hysteresis in the underlying hard-magnetic particles. The proposed constitutive model is thus developed following the generalized standard materials framework, which necessitates suitable definitions of the energy density and the dissipation potential. Moreover, the proposed model is designed to recover several well-known homogenization results (and bounds) in the purely mechanical and purely magnetic limiting cases. The magneto-mechanical coupling response of the model, in turn, is calibrated with the aid of numerical homogenization estimates under symmetric cyclic loading. The performance of the model is then probed against several other numerical homogenization estimates considering various magneto-mechanical loading paths other than the calibration loading path. Very good agreement between the macroscopic model and the numerical homogenization estimates is observed, especially for stiff to moderately-soft matrix materials. An important outcome of the numerical simulations is the independence of the current magnetization to the stretch part of the deformation gradient. This is taken into account in the model by considering an only rotation-dependent remanent magnetic field as an internal variable. We further show that there is no need for an additional mechanical internal variable. Finally, the model is employed to solve macroscopic boundary value problems involving slender $h$-MRE structures and the results match excellently with experimental data from literature. Crucial differences are found between uniformly and non-uniformly pre-magnetized $h$-MREs in terms of their pre-magnetization and the associated self-fields.
\end{abstract}

Keywords: Magnetorheological Elastomers, Hard Magnetic, Finite-strains, Magnetic Dissipation, Microstructural Modeling, Magneto-mechanical coupling, Homogenization, Finite-Elements

\section{Introduction}

Rare earth-based, hard (permanent) magnetic NdFeB particle-filled magnetorheological elastomers (MREs) have been recently explored towards their potential applications in magnetic soft robots (Kim et al., 2018; Zhao et al., 2019; Ren et al., 2019; Alapan et al., 2020), microfluidic separators (Hilber and Jakoby, 2012; Royet et al., 2017; Zhou et al., 2020), and bio-inspired magnetic sensors (Kaidarova et al., 2018) among other innovative applications. In contrast to traditional soft magnetic iron particle-filled MREs, denoted henceforth as $s$-MREs, (Danas et al., 2012; Bodelot et al., 2017), NdFeB particle-filled hard MREs, denoted as $h$-MREs, exhibit remanent magnetization. Because of this feature, $h$-MREs may be actuated by much smaller magnetic fields in comparison with iron-based MREs (Kim et al., 2018; Zhao et al., 2019; Ren et al., 2019; Alapan et al., 2020). In turn, in the works of Kim et al. (2018) and Alapan et al. (2020), the initial magnetization is achieved through strong magnetic fields during the 3D printing process.

The fabrication of magneto-active particle-filled MREs was initiated by Rigbi and Jilkén (1983), who cured soft iron particles in a natural rubber matrix and consequently investigated the mechanical properties of the composite under applied magnetic field. Since then, numerous fabrication techniques and experimental probes for $s$-MREs have been reported (Jolly et al., 1996; Ginder et al., 1999, 2000; Lokander and Stenberg, 2003; Danas et al., 2012; Bodelot et al., 2017). NdFeB particles bonded in polymers, on the other hand, were initially fabricated to replace the traditional permanent magnets in various applications (Garrell et al., 2003). More recently, 3D printing has been exploited for the creation of permanent magnets with a desired distribution of the magnetic field (Huber et al., 2017; Taylor et al., 2019). Due to the aim of replacing hard permanent magnets, such "polymer bonded magnets" are mechanically stiff by design. In contrast to these mechanically stiff magnets, $h$-MREs are based on NdFeB particles suspended in rather soft matrix materials like polydimethylsiloxane (PDMS) (Linke et al., 2016; Schümann and Odenbach, 2017; Kalina et al., 2017; Sánchez et al., 2018). However, $h$-MREs made of such soft elastomers with shear modulus $G \sim 0.04-0.001 \mathrm{MPa}$

\footnotetext{
* Corresponding author

Email addresses: dipayan.mukherjee@polytechnique.edu (Dipayan Mukherjee), matthias.rambausek@polytechnique.edu (Matthias Rambausek), konstantinos.danas@polytechnique.edu (Kostas Danas)
} 
(Schümann and Odenbach, 2017) are often too soft to be used as structural elements. Moreover, the interactions of the strongly magnetic $\mathrm{NdFeB}$ particles typically lead to considerable damage of the matrix. To avoid such effects, recent investigations on the coupled structural response of $h$-MREs use a moderately soft commercially available Sylgard 184 (10:1) PDMS matrix (Kim et al., 2018; Zhao et al., 2019) with a mechanical shear modulus in the range of $G \sim$ $0.4-1.2 \mathrm{MPa}$ (Choi and Rogers, 2003; Johnston et al., 2014; Park et al., 2018).

On the other hand, the particles are practically rigid by comparison to the elastomer matrix and thus can only undergo rotation but not deformation in the context of the MRE composites under consideration. Their magnetic response, however, is more complex and involves several scales. Specifically, the size of the magnetic particles employed in both magnetically hard and soft MREs is in the order of several microns. Hence, they are considerably smaller than the leading dimensions of typical "macroscopic" samples. In particular, the commercially available (both, mechanically and magnetically) isotropic NdFeB particles (also known as MQP-S particles) have a median diameter of $10-30 \mu m$, while the dimensions of the fabricated $h$-MRE composites typically is in the order of $1-100 \mathrm{~mm}$ (Kim et al., 2018; Zhao et al., 2019). Moreover, the hard-magnetic particles are polycrystalline in nature, having randomly-oriented grains of $\sim 50 \mathrm{~nm}$ size (Huber et al., 2019). As a consequence, the ferromagnetic hysteresis in these hard magnetic particles occurs at the nanometer scale since it results from the domain wall motions and their pinning/unpinning at the grain boundaries (Livingston, 1981). This implies that a complete analysis of all physical mechanisms requires the consideration of at least three scales ranging from the nano- to the millimeter scale, which makes the problem highly intractable in an analytical sense.

In this work, we will focus on a more continuum approach with the goal of providing an explicit analytical model at the macroscopic scale. In particular, the continuum magneto-elastic modeling traces its way back to Tiersten (1964, 1965) and Brown (1966). Since then, a set of different continuum models and formulations for the coupled magnetomechanical response of magnets has been proposed (see the monographs by Hutter and van de Ven (1978); Kovetz (2000)). More recently, the magneto-elastic theory has been revisited and modernized for the modeling of $s$-MREs by Dorfmann and Ogden $(2003,2004)$ and Kankanala and Triantafyllidis (2004). A continuum model for the anisotropic $s$-MREs is subsequently proposed by Danas et al. (2012). These so-called "top-down" phenomenological models are devised from macroscopic experimental observations. In all those models, the ferromagnetic response of the particles and the composite is modeled, rather efficiently, in a continuum manner circumventing the processes at the scale of domain wall motion.

On the other hand, "bottom-up" approaches allowing to model the macroscopic response of $s$-MREs have been developed based on the (variational) homogenization of local microscopic potentials associated with the two individual phases of the composite. For these methods, the decision on the relevant scales is of great theoretical and practical importance. If one is interested in understanding the physical nature of ferromagnetism at the nanometer or micrometer scale of the particles themselves and the corresponding domain wall motion, one appropriate framework is that of micromagnetics which goes back to Brown (1963) (but see important works along these lines by James and Kinderlehrer (1993) and DeSimone and James (2002)). This theory has been very recently extended in the context of magnetomechanics of elastomer composites by Keip and Sridhar (2018) in a two-dimensional numerical setting. Unfortunately, it is extremely hard to obtain three-dimensional analytical "explicit" models for domain wall motion at that nanoscale that also take into account particle interactions at the $h$-MRE scale. In turn, the numerical resolution of magnetic domains is computationally extremely demanding and for the moment it has mainly been achieved in two-dimensions and at the scale of a few particles.

By contrast, when the magnetic particles are much larger than the typical magnetic domain scale (roughly the grain size), as is the case in the present study, a phenomenological description of the magnetic response of the particles is often sufficiently accurate (Mukherjee and Danas, 2019). This holds in particular when the particles are themselves small in comparison with the actual scale of interest, i.e. the macroscopic scale. Hence, the majority of works on homogenization in the context of MREs start from the particle scale (microns) which is then often referred to as microscale. Analytical homogenization estimates for the "effective response" of mechanically incompressible, soft magnetic composites are provided by Ponte Castañeda and Galipeau (2011) and Lefèvre et al. (2017), while the numerical homogenization estimates for the same are provided by Kalina et al. (2016) and Danas (2017). Moreover, Keip and Rambausek (2016, 2017 ) developed a multi-scale $\left(\mathrm{FE}^{2}\right)$ computational framework for $s$-MREs. However, none of the analytical or numerical homogenization frameworks provide an explicit macroscopic model that can be readily applied to a macroscopic boundary value problem. Thus, "microstructure-guided" phenomenological models have been proposed recently by Lefèvre et al. (2019) and Mukherjee et al. (2020) and benchmarked against the analytical homogenization model of Lefèvre et al. (2017) with good overall agreement and almost identical results in certain limiting cases. An alternative approach towards such microstructurally-guided models has been developed recently by Kalina et al. (2020), which however, requires expensive computations in order to fit a comparatively large set of model parameters. However, all models in those studies are concerned with $s$-MREs involving no dissipation, neither mechanical nor magnetic.

From the viewpoint of dissipative MREs, Saxena et al. (2013, 2014) and Hossain et al. (2015) have included the effect of viscoelasticity in a top-down approach to analyze the impact of cyclic loading and curing in the response and fabrication of MREs. By contrast, following a bottom-up approach, Garcia-Gonzalez and Hossain (2020) provided a micromechanical model based on dipole interactions for magneto-viscoelastic materials. Additional contributions including the effect of diffusion may also be mentioned in the context of dissipative magneto-rheological gels (Garcia-Gonzalez and Landis, 
2020). In all those studies, the dissipation results from the mechanical part, while the magnetic part is purely energetic. As a consequence, none of the above-mentioned models can be used to model the dissipative magnetic response of $h$-MREs, which is the focus of the present work.

In this regard, the thermodynamically consistent modeling of the remanent magnetization is typically carried out via introducing a remanent internal variable. Such thermodynamic formalism has been first introduced to describe the remanent polarization in ferroelectric ceramics (Huber et al., 1999; Huber and Fleck, 2001; McMeeking and Landis, 2002; Landis, 2002; Klinkel, 2006). Subsequently, this framework has been extended to ferromagnetic materials (Linnemann et al., 2009; Mukherjee and Danas, 2019). All the aforementioned works, however, have been developed in a small strain setting. Miehe et al. (2011) provide a general numerical implementation procedure for the simulation of dissipative electromagneto-elastic materials at small strains. Based on that, Rosato and Miehe (2014) proposed a "top-down" approach towards modeling the remanent polarization in ferroelectricity at finite strains. Therein, the (remanent) deformation due to the remanent polarization is introduced explicitly to be a symmetric rotation-free tensor that encodes transverse isotropy with preferred direction aligned to the polarization.

Turning now to the modeling of $h$-MREs, in a top-down approach, Zhao et al. (2019) proposed a purely energetic torque-based model with no self-field magneto-mechanical coupling (i.e. no coupling in the sense of particle interactions) to describe the magneto-mechanical response of uniformly pre-magnetized $h$-MREs. Given its non-dissipative character, this model is valid for fairly small applied magnetic fields around a pre-magnetization state. Furthermore, the premagnetization distribution needs to be postulated or resolved separately with other modeling tools. Despite those limitations, that model has been shown to reproduce successfully corresponding experimental results on uniformly premagnetized slender beams under small applied magnetic fields up to $\sim 50 \mathrm{mT}$.

On the other hand, recent numerical simulations of Kalina et al. (2017) in $h$-MREs revealed, although not directly, some interesting effects relating magnetic dissipation with drastic microstructural rearrangements when subjected to cyclic magnetic loads of finite amplitude. Yet, an appropriate incremental homogenization formalism for microstructured $h$-MREs that allows for the determination of effective properties from microstructural simulations is still lacking. In this regard, we rely on incremental variational principles (Ortiz and Stanier, 1999) which render an incremental variational homogenization framework (Miehe, 2002; Miehe et al., 2002) for the computation of the effective response of dissipative coupled composites that will serve as the basis of the present numerical homogenization analysis. Moreover, Danas (2017) has shown that the numerical homogenization for magneto-elastic (but also electro-elastic) composites necessitates suitable augmentations to the variational homogenization problem in order to obtain the effective magneto-mechanical response due to pure particle-particle interactions, which is free from geometric shape effects of the MRE sample (Keip and Rambausek, 2017).

In this regard and to the best knowledge of the authors, there exists no bottom-up or top-down analytical model up-to-date that takes into account the magnetic dissipation on the magneto-mechanical response of $h$-MREs at finite strains and arbitrary proportional and non-proportional magnetic fields. In view of these considerations and particularly the fact that very soft $h$-MREs may lead to drastic particle rearrangements and microstructural damage under large magnetic fields (see e.g., Schümann and Odenbach (2017)), the goal of the present work is to propose a model for moderately soft $h$-MREs, which has the following desired properties. First, all constitutive relations describing the magneto-mechanical dissipative response of the $h$-MREs should be explicit and analytical. This allows for an efficient numerical implementation of the model in user material subroutines to solve macroscopic boundary value problems of any type. Second, the model should also be derived in a thermodynamically consistent manner, so that it can deal properly with the vast range of possible loading conditions (e.g., magnetic, mechanical, magneto-mechanical, monotonic, non-proportional or cyclic) without risking to have negative dissipation. This is achieved by use of a convex dissipation potential within the framework of generalized standard materials (Halphen and Son Nguyen, 1975). Third, it is desirable that the proposed model recovers existing rigorous homogenization results in limiting cases of interest. For instance, in the energetic limit, where the dissipation vanishes, the model should recover the non-dissipative magnetic and mechanical homogenization estimates of Lefèvre et al. (2017). Fourth, the model should reproduce with only a handful of calibration parameters the effective coupled dissipative response of $h$-MREs obtained by rigorous numerical representative volume element (RVE) calculations. Such calculations are carried out also in this study for the first time. Finally, the complete model should be versatile enough to allow easy calibration to experiments even when the individual responses of the constituents are not known.

The paper is organized as follows. Section 2 summarizes the fundamental equations of magnetostatics and continuum mechanics for the present case. Next, Section 3 introduces a thermodynamically consistent model for isotropic, incompressible $h$-MREs beginning with a detailed description of the basic assumptions, followed by the derivation of the constitutive relations by employing Coleman-Noll arguments and expressing the necessary properties of the energetic and dissipation potentials. Next, specific choices for the energy density function and the dissipation potential are detailed in Section 4. Subsequently, Section 5 presents an incremental variational homogenization framework that is employed to compute the effective response of the $h$-MREs via realizing the microscopic fields explicitly, followed by computing their volume averages. These results will then provide the data for the actual calibration of the proposed macroscopic model in Section 6 . In the same section, the fitted model is then probed against the numerical homogenization estimates for various non-proportional magneto-mechanical loading paths in order to demonstrate its predictive capabilities. The practical modeling capabilities of the proposed macroscopic model are assessed in Section 7, where we solve macroscopic boundary 
value problems to simulate the experiments of Zhao et al. (2019), involving a uniformly pre-magnetized $h$-MRE cantilever beam. Furthermore, motivated by the recent experimental work of Ren et al. (2019), we study the bending response of idealized slender non-uniformly pre-magnetized $h$-MRE beams subjected to a transverse magnetic field. Therein, we show the importance of using a complete model for $h$-MREs together with the modeling of the surrounding air. We close the main part with concluding remarks are provided in Section 8. Finally, in Appendix A, we provide an alternative equivalent Eulerian formulation of the problem and in Appendix B, we detail explicit expressions for the derivatives of the new energetic-remanent invariants used in the present work.

\section{Preliminary definitions}

We consider a deformable magneto-active solid, which occupies a volume $\mathcal{V}_{0} \in \mathbb{R}^{3}\left(\mathcal{V} \in \mathbb{R}^{3}\right)$ in the reference (current) configuration with the boundary $\partial \mathcal{V}_{0}(\partial \mathcal{V})$ and outward unit normal $\mathcal{N}(\mathbf{n})$. The reference and current configurations are related through the one-to-one deformation map $\boldsymbol{y}(\mathbf{X})$, while $\boldsymbol{y}(\mathbf{X})=\mathbf{X}$ for all $\mathbf{X} \in \mathbb{R}^{3} \backslash \mathcal{V}_{0}$, except in the vicinity of the solid. We henceforth assume that $\boldsymbol{y}(\mathbf{X})$ is continuous everywhere and twice continuously differentiable except at the solid-air interface or the interfaces between different phases in the material. The deformation gradient is defined as $\mathbf{F}=\operatorname{Grad} \boldsymbol{y}$, where Grad denotes the gradient operator with respect to $\mathbf{X}$, which is the reference position vector of a material point in $\mathcal{V}_{0}$. In addition, the reference density of the solid $\rho_{0}$ is related to the current density $\rho$ by $\rho=J \rho_{0}$, where $J=\operatorname{det} \mathbf{F}>0$.

In the absence of electrodynamic effects and vanishing electric current in $\mathcal{V}$, the local Eulerian magnetic field $\mathbf{b}$ is divergence free and the local $\mathbf{h}$ field is curl free, i.e.

$$
\begin{aligned}
& \operatorname{curlh}=0 \quad \text { in } \mathbb{R}^{3}, \quad \llbracket \mathbf{h} \rrbracket \times \mathbf{n}=0 \quad \text { on } \partial \mathcal{V}, \\
& \operatorname{divb}=0 \quad \text { in } \mathbb{R}^{3}, \quad \llbracket \mathbf{b} \rrbracket \cdot \mathbf{n}=0 \quad \text { on } \partial \mathcal{V} .
\end{aligned}
$$

The current magnetization $\mathbf{m}$ is defined by the constitutive relation

$$
\mathbf{b}=\mu_{0}(\mathbf{h}+\mathbf{m}) \quad \text { in } \mathbb{R}^{3},
$$

where $\mu_{0}$ is the permeability of vacuum. In principle, any one of $\mathbf{b}$ or $\mathbf{h}$ (but not $\mathbf{m}^{1}$ ) may be selected as a primary variable. Within this contribution, we choose to work with $\mathbf{h}$ as a primary variable, whereas $\mathbf{b}$ will be obtained via the proposed constitutive laws. In addition, $\mathbf{m}$ will be used for illustrating a number of results and thus is an auxiliary variable obtained via $(2.3)$.

In the case of finite deformation of a magnetic solid, the Lagrangian counterparts of the Eulerian fields $\mathbf{b}$ and $\mathbf{h}$ can be obtained by a pull-back operation from $\mathcal{V}$ to $\mathcal{V}_{0}$ and are denoted by $\mathbf{B}$ and $\mathbf{H}$, respectively, such that (Dorfmann and Ogden, 2003, 2004; Kankanala and Triantafyllidis, 2004)

$$
\mathbf{B}=J \mathbf{F}^{-1} \mathbf{b}, \quad \mathbf{H}=\mathbf{F}^{T} \mathbf{h} .
$$

The Lagrangian $\mathbf{H}$ and $\mathbf{B}$ satisfy the the referential versions of (2.1) and (2.2), which read as

$$
\begin{aligned}
\operatorname{Curl} \mathbf{H}=0 & \text { in } \mathbb{R}^{3}, & & \llbracket \mathbf{H} \rrbracket \times \mathcal{N}=0 \quad \text { on } \partial \mathcal{V}_{0}, \\
\operatorname{DivB}=0 & \text { in } \mathbb{R}^{3}, & & \llbracket \mathbf{B} \rrbracket \cdot \mathcal{N}=0 \quad \text { on } \partial \mathcal{V}_{0},
\end{aligned}
$$

where the operators "Curl" and "Div" represent, respectively, the curl and divergence with respect to the referential X.

The mechanical balance laws consist of the linear and angular momentum balances. In absence of any body forces, the mechanical linear momentum balance laws for a magneto-elastic solid read as

$$
\begin{aligned}
& \operatorname{Div} \mathbf{S}=0 \quad \text { in } \mathbb{R}^{3}, \quad \llbracket \mathbf{S} \rrbracket \cdot \mathcal{N}=0 \quad \text { on } \partial \mathcal{V}_{0}, \\
& \operatorname{div} \boldsymbol{\sigma}=0 \quad \text { in } \mathbb{R}^{3}, \quad \llbracket \boldsymbol{\sigma} \rrbracket \cdot \mathbf{n}=0 \quad \text { on } \partial \mathcal{V},
\end{aligned}
$$

in the Lagrangian and Eulerian configurations, respectively. The stresses $\mathbf{S}$ and $\boldsymbol{\sigma}$ in (2.7) and (2.8) are the total ${ }^{2}$ first Piola-Kirchhoff and total Cauchy stress, respectively, which are related via

$$
\mathbf{S}=J \boldsymbol{\sigma} \mathbf{F}^{-T}
$$

Finally, the local form of the mechanical angular momentum balance is simply given by

$$
\boldsymbol{\sigma}=\boldsymbol{\sigma}^{T} \text { and } \quad \mathbf{S F}^{T}=\mathbf{F S}^{T}
$$

\footnotetext{
${ }^{1}$ The $\mathbf{m}$ alone cannot describe entirely a magnetic boundary value problem since the magnetic fields $\mathbf{h}$ and $\mathbf{b}$ are non-zero even inside non-magnetic phases (i.e. with $\mu_{0}$ finite) but have $\mathbf{m}=\mathbf{0}$. In former studies, such as that of Danas et al. (2012) that $\mathbf{m}$ was one of the primary variables additionally $\mathbf{b}$ was also present in the global formulation.

${ }^{2}$ In the sense that they account for mechanical and magneto-mechanical contributions. Hence, they are also present in air and vacuum.
} 
in the current and the reference configurations, respectively (Kankanala and Triantafyllidis, 2004). We note that several "classical" formulations of magneto-elasticity consider applied magnetic body forces and body couples, which are taken into consideration in the linear and angular momentum balance laws, respectively (Brown, 1966; Pao and Hutter, 1975; Hutter and van de Ven, 1978). Nevertheless, in this paper we follow the approach of Kovetz (2000) and Kankanala and Triantafyllidis (2004), where the (electro)magnetic energy flux is accounted for in terms of the Poynting vector. This together with the balance of energy, the entropy inequality and the Coleman-Noll argument avoids the postulation of magnetic forces and instead yields the concept of "total stress" which is used throughout this contribution.

\section{Thermodynamics and constitutive properties}

This section develops a thermodynamically consistent, microstructurally-guided constitutive modeling framework for $h$-MREs. We first discuss the choice for the magnetic internal variable and the associated kinematic assumptions, which are at the heart of the model and subsequently establish a $\mathbf{F}-\mathbf{H}$ constitutive modeling framework. The objectivity and material symmetry conditions for the present case of isotropic $h$-MREs are then set, wherein the definition of the internal variables and their kinematics play a pivotal role. This allows then to define proper energetic and remanent mechanical, magnetic and magneto-mechanical invariants.

\subsection{Internal variable for magnetic dissipation}

A thermodynamically consistent model for any dissipative material may be constructed through the definition of a finite number of internal variables, which reflect the irreversible processes the material undergoes under external loads. In this regard, one of the principal differences between $h$-MREs and $s$-MREs is the underlying magnetic dissipation of the filler particles (e.g. NdFeB) in the former. Due to the finite strains and the magneto-mechanical coupling, upon cyclic magnetic loading, the response of the $h$-MRE composite exhibits both magnetic and mechanical (due to magneto-mechanical coupling) hysteresis.

In the present work, we will show by extensive assessment with numerical RVE simulations in Sections 6.2 .1 and 6.2.2 that only one internal magnetic vectorial variable suffices to describe both the magnetic and the mechanical (due to coupling) dissipation in the $h$-MRE in the case of moderately soft to hard polymer matrices. This is a mere constitutive choice and does not constitute a unique way to address this coupled dissipative effect ${ }^{3}$.

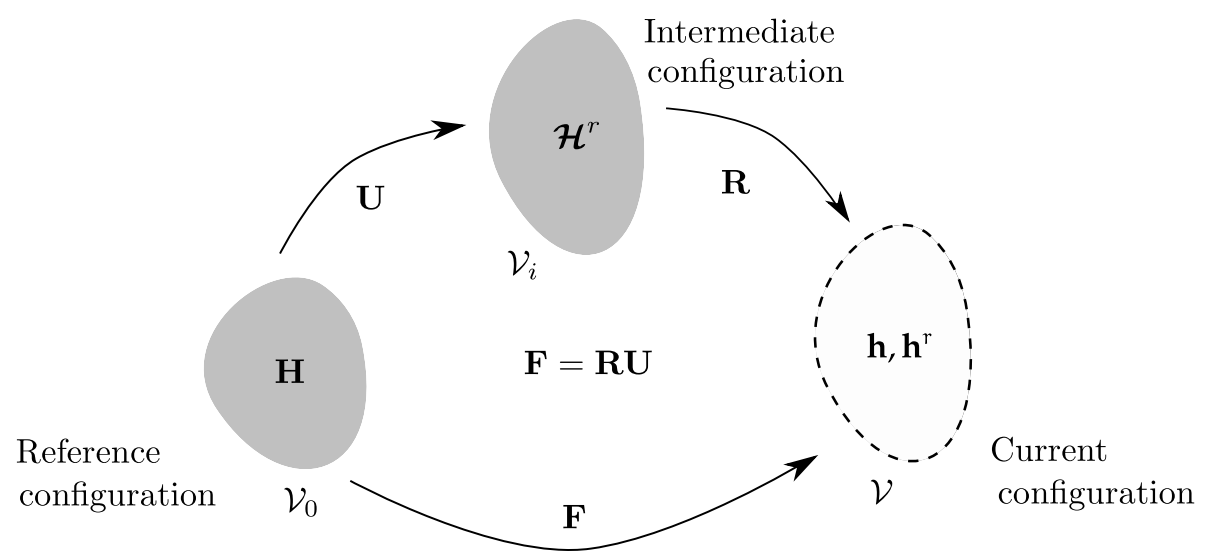

Figure 1: Definition of the independent internal variable $\boldsymbol{H}^{r}$ in the intermediate stretch-free configuration $\mathcal{V}_{i}$. The components $\mathbf{U}$ and $\mathbf{R}$ denote the stretch tensor and the rotational component, respectively, of the deformation gradient $\mathbf{F}$.

Specifically, we propose to introduce a magnetic internal variable, the remanent field $\mathcal{H}^{r}$ in the stretch-free, intermediate configuration $\mathcal{V}_{i}$, as shown in Fig. 1. The central assumption behind this "choice" concerning the macroscopic model is that the average magnetization of the composite and thus magnetic dissipation is affected by macroscopic rotations but is independent of macroscopic stretches. The range of validity of this assumption, which will be assessed in detail in Sections 6.2.1 and 6.2.2, depends mainly on the shear modulus of the matrix phase, given that the particles are almost mechanically rigid and thus do not deform but may rotate, whereas the bulk modulus of the matrix phase is very large giving rise to a quasi-incompressible macroscopic response of the $h$-MRE. In fact, the shear modulus of the matrix controls the capability of the magnetized particles to rotate upon application of a magnetic load that is non-aligned with their magnetization vector. In a soft polymeric matrix, the particles rotate mechanically but also evolve their magnetic state via dissipative mechanisms (such as domain wall motions) in order to align their magnetization with the externally applied magnetic field (Kalina et al., 2017). The softer the matrix the more the rotations prevail over the magnetic dissipation mechanisms. By contrast, when the shear modulus of the matrix has a moderate value (e.g. greater than

\footnotetext{
${ }^{3}$ Works by McMeeking and Landis (2005); McMeeking et al. (2007); Rosato and Miehe (2014) in the context of ferroelectricity, a somewhat similar problem, use both mechanical and polarization internal variables that are eventually related via ad-hoc constitutive relations.
} 
$\sim 0.15 \mathrm{MPa}$ ), local particle rotations are much less pronounced and dissipative processes dominate the response of the $h$-MRE, while any rotations of the magnetized particles follow approximately the overall macroscopic rotation. This last case corresponds well to actual, fabricated $h$-MREs in the literature (see for instance Zhao et al. (2019) and Ren et al. (2019)). In particular, we will show in the context of Figs. 7 and 8 that a purely tensile or shear mechanical load of a remanently magnetized $h$-MRE does not change the amplitude of the effective magnetization but only its direction which follows the macroscopic average rotations. This result is in direct connection with an internal remanent field lying in the intermediate and not in the undeformed configuration.

In view of this discussion, use of the standard polar decomposition $\mathbf{F}=\mathbf{R U}$ directly implies that the reference (in $\mathcal{V}_{0}$ ) and current (in $\mathcal{V}$ ) remanent fields, $\mathbf{H}^{r}$ and $\mathbf{h}^{r}$, respectively are functions of $\mathcal{H}^{r}$ and are given by

$$
\mathbf{h}^{r}=\mathbf{R} \mathcal{H}^{r}, \quad \mathbf{H}^{r}=\mathbf{U H}^{r}
$$

whereby the following standard decompositions also hold (Mukherjee and Danas, 2019)

$$
\mathbf{h}=\mathbf{h}^{e}+\mathbf{h}^{r}, \quad \mathbf{H}=\mathbf{H}^{e}+\mathbf{H}^{r} .
$$

Here, $\mathbf{h}$ and $\mathbf{h}^{e}$ are the Eulerian total and energetic $h$-fields, while $\mathbf{H}$ and $\mathbf{H}^{e}$ are the corresponding Lagrangian ones. The introduction of $\mathcal{H}^{r}$ in the intermediate configuration $\mathcal{V}_{i}$ further implies that the Eulerian $\mathbf{h}^{r}$ is stretch-free function of $\mathbf{R}$ and $\mathcal{H}^{r}$, while the Lagrangian $\mathbf{H}^{r}$ is a function of $\mathbf{U}$ and $\mathcal{H}^{r}$.

Obviously, the propositions (3.1) remain open to further refinements if required by the problem at hand. Nonetheless, we will show in the results presented in Section 6 that the single internal variable $\mathcal{H}^{r}$ is sufficient to achieve very good agreement with the corresponding numerical RVE simulations under a wide range of cyclic and non-aligned loading conditions.

\subsection{Clausius-Duhem inequality and generic constitutive relations}

In this section, we derive the constitutive relations for the $h$-MREs in the reference configuration. The (local) Clausius-Duhem inequality, which is derived from the (global) entropy imbalance, leads to a thermodynamically consistent constitutive framework for dissipative ferro-electric/magnetic materials (McMeeking and Landis, 2002; Landis, 2002; Klinkel, 2006; Rosato and Miehe, 2014). The present work is concerned with isothermal thermodynamic processes that involve zero heat flux and local variation in the temperature. Thus, the Clausius-Duhem inequality for a magnetoelastic solid is given by

$$
\mathbf{S}: \dot{\mathbf{F}}-\mathbf{B} \cdot \dot{\mathbf{H}}-\dot{W} \geq 0
$$

where $(\dot{)})$ represents the material time derivative. The energy density $W=W\left(\mathbb{C}, \mathbf{H}, \mathcal{H}^{r}\right)$ is expressed in terms of the right Cauchy-Green tensor $\mathbb{C}=\mathbf{F}^{T} \mathbf{F}$, the referential $\mathbf{H}$ and the internal variable $\mathcal{H}^{r}$. Such a definition of $W$ in terms of $\mathbb{C}$ ensures material frame indifference. Expanding subsequently $\dot{W}$ in terms of its arguments, we rephrase (3.3) as

$$
\left[\mathbf{S}-2 \mathbf{F} \frac{\partial W}{\partial \mathbb{C}}\right]: \dot{\mathbf{F}}-\left[\mathbf{B}+\frac{\partial W}{\partial \mathbf{H}}\right] \cdot \dot{\mathbf{H}}-\frac{\partial W}{\partial \mathcal{H}^{r}} \cdot \dot{\mathcal{H}}^{r} \geq 0 .
$$

Given the arbitrariness of the rates $\dot{\mathbf{F}}$ and $\dot{\mathbf{H}}$, the standard Coleman-Noll-Gurtin arguments lead to the constitutive relations in the reference configuration

$$
\mathbf{S}=2 \mathbf{F} \frac{\partial W}{\partial \mathbb{C}}, \quad \mathbf{B}=-\frac{\partial W}{\partial \mathbf{H}}
$$

Since the derivative of a scalar-valued tensor function with respect to a symmetric tensor is symmetric, the Cauchy stress $\boldsymbol{\sigma}=J^{-1} \mathbf{S F}^{T}$ computed from $(3.5)_{1}$ remains identically symmetric. It thus satisfies the angular momentum balance (Kankanala and Triantafyllidis, 2004; Suo et al., 2008; Rosato and Miehe, 2014) as detailed in Appendix A.

The remaining terms in (3.4) lead to the dissipation inequality, which reads

$$
\mathcal{B}^{r} \cdot \dot{\mathcal{H}}^{r} \geq 0, \quad \mathcal{B}^{r}=-\frac{\partial W}{\partial \mathcal{H}^{r}}
$$

Of course, the remanent field $\mathcal{B}^{r}$ is the conjugate force quantity to $\mathcal{H}^{r}$ and is also defined in the intermediate configuration (see Fig. 1). Using the generalized standard material framework (Halphen and Son Nguyen, 1975), one can readily write

$$
\frac{\partial W}{\partial \mathcal{H}^{r}}+\frac{\partial D}{\partial \dot{\mathcal{H}}^{r}}=0 \quad \Longrightarrow \quad \mathcal{B}^{r}=\frac{\partial D}{\partial \dot{\mathcal{H}}^{r}},
$$

where $D\left(\mathbb{C}, \mathbf{H}, \mathcal{H}^{r}, \dot{\mathcal{H}}^{r}\right) \geq 0$ is the dissipation potential. We note that the present hybrid formulation leads to the primary constitutive relations (3.5) in the reference configuration, whereas the remanent constitutive relations (3.6) $)_{2}$ and (3.7) are defined in the intermediate configuration. One can develop the full constitutive framework in the intermediate configuration (see e.g., (Rosato and Miehe, 2014)), however, the reference primary variables lead to familiar stress measures like $\mathbf{S}$ and also facilitate the numerical computations (Danas, 2017; Lefèvre et al., 2017). An equivalent framework in terms of Eulerian quantities is provided in Appendix A. 
Remark 1. It is notoriously troublesome to express $\mathbf{S}$ and $\boldsymbol{\sigma}$ explicitly in terms of mechanical and magnetic stress contributions (McMeeking and Landis, 2005). Nevertheless, some insight can be gained when the referential $\mathbf{H}_{\text {and }} \boldsymbol{\mathcal { H }}^{r}$ are expressed in terms of their current counterparts $\mathbf{h}=\mathbf{h}(\mathbf{F}, \mathbf{H})$ and $\mathbf{h}^{r}=\mathbf{h}^{r}\left(\mathbf{R}(\mathbf{F}), \mathcal{H}^{r}\right)$, respectively. We consider an energy density $W\left(\mathbf{F}, \mathbf{H}, \mathcal{H}^{r}\right)=W\left(\mathbb{C}, \mathbf{H}, \mathcal{H}^{r}\right)$ with $\mathbb{C}=\mathbf{F}^{T} \mathbf{F}$ that has an equivalent form $w\left(\mathbb{B}, \mathbf{h}, \mathbf{h}^{r}\right)$ with $\mathbb{B}=\mathbf{F} \mathbf{F}^{T}$, i.e. $W\left(\mathbf{F}, \mathbf{H}, \boldsymbol{\mathcal { H }}^{r}\right) \equiv w\left(\mathbb{B}, \mathbf{h}, \mathbf{h}^{r}\right)$. In addition, following standard knowledge in magneto-elasticity, we decompose the free energy density as $w\left(\mathbb{B}, \mathbf{h}, \mathbf{h}^{r}\right)=\rho_{0} \psi\left(\mathbb{B}, \mathbf{h}, \mathbf{h}^{r}\right)-\left(\mu_{0} / 2\right) J \mathbf{h} \cdot \mathbf{h}$ with $\psi$ being the Helmholtz free energy associated with the $h$-MRE (to be defined in Section 4). Then, the equivalent representation of $\boldsymbol{\sigma}$ in terms of $w$ is

$$
\begin{aligned}
\boldsymbol{\sigma}=\frac{1}{J} \frac{\partial W}{\partial \mathbf{F}} \mathbf{F}^{T} & =\frac{2}{J}\left[\frac{\partial w}{\partial \mathbb{B}}\right]_{\mathbf{h}, \mathbf{h}^{r}} \mathbb{B}+\frac{1}{J}\left(\left[\frac{\partial w}{\partial \mathbf{h}^{r}}\right]_{\mathbf{F}, \mathbf{h}} \cdot \frac{\partial \mathbf{h}^{r}}{\partial \mathbf{F}}\right) \mathbf{F}^{T}+\frac{1}{J}\left(\left[\frac{\partial w}{\partial \mathbf{h}}\right]_{\mathbf{F}, \mathbf{h}^{r}} \cdot \frac{\partial \mathbf{h}}{\partial \mathbf{F}}\right) \mathbf{F}^{T} \\
& =\underbrace{\frac{2 \rho_{0}}{J}\left[\frac{\partial \psi}{\partial \mathbb{B}}\right]_{\mathbf{h}, \mathbf{h}^{r}}}_{\boldsymbol{\sigma}^{e}} \mathbb{B}+\underbrace{\frac{\rho_{0}}{J}\left\{\left(\left[\frac{\partial \psi}{\partial \mathbf{h}^{r}}\right]_{\mathbf{F}, \mathbf{h}} \otimes \mathbf{R}^{T} \mathbf{h}^{r}\right): \frac{\partial \mathbf{R}}{\partial \mathbf{F}}\right\} \mathbf{F}^{T}}_{\boldsymbol{\sigma}^{r}}+\underbrace{\left(\mathbf{h} \otimes \mathbf{b}-\frac{\mu_{0}}{2}|\mathbf{h}|^{2} \mathbf{I}\right)}_{\boldsymbol{\sigma}^{\operatorname{maxw}}} .
\end{aligned}
$$

In this expression ${ }^{4}$, one may then define, with a relative abuse of notation, the mechanical part ${ }^{5}$ of the stress as $\boldsymbol{\sigma}^{\text {mech }}=$ $\boldsymbol{\sigma}^{e}+\boldsymbol{\sigma}^{r}$. The first and last terms in (3.8) are identical to those already obtained in the context of the non-remanent, $s$-MREs (Kankanala and Triantafyllidis, 2004; Danas, 2017), while, the remanent stress contribution $\boldsymbol{\sigma}^{r}$ appears because of the remanent internal variable $\mathcal{H}^{r}$. By its definition, the $\boldsymbol{\sigma}^{\text {maxw }}$ is non-zero even in the absence of a material (i.e., if $\psi=0)$ if the magnetic fields are non-zero. In turn, the mechanical stress, $\boldsymbol{\sigma}^{\text {mech }}$ includes both the energetic and remanent contributions, since they can both exist in the absence of an applied external magnetic field. We note further that only the symmetry of the "total" Cauchy stress $\boldsymbol{\sigma}$ is guaranteed by $W\left(\mathbb{C}, \mathbf{H}, \boldsymbol{H}^{r}\right)$, whereas $\boldsymbol{\sigma}^{\text {mech }}$ and $\boldsymbol{\sigma}^{\text {maxw }}$ are, in general, not symmetric.

\subsection{General properties of the free energy density and the dissipation potential}

Before we proceed to specific constitutive propositions, we discuss first the constraints that need to be imposed on the energetic and dissipation potentials, $W$ and $D$, respectively, in order to ensure (a) an even magneto-mechanical coupling, (b) material frame indifference, (c) isotropic material symmetry and (d) positive dissipation (see $\left.(3.6)_{1}\right)$.

We recall here that material objectivity and symmetry conditions are well-known for $s$-MREs (Kankanala and Triantafyllidis, 2004; Dorfmann and Ogden, 2004). However, as shown in Fig. 1, the present model for $h$-MREs introduces a remanent internal variable that is insensitive to the stretch $\mathbf{U}$, leading to non-familiar magneto-mechanical invariants and rates. Hence, this section focuses on stating explicitly all the aforementioned constraints in the context of isotropic $h$-MREs.

Even magneto-mechanical coupling. The magneto-mechanical energy density $W$ and dissipation potential $D$ must be exactly the same when both $\mathbf{H}$ and $\mathcal{H}^{r}$ change simultaneously sign. This condition reads

$$
W\left(\mathbb{C},-\mathbf{H},-\mathcal{H}^{r}\right)=W\left(\mathbb{C}, \mathbf{H}, \mathcal{H}^{r}\right), \quad D\left(\mathbb{C},-\mathbf{H},-\mathcal{H}^{r},-\dot{\mathcal{H}}^{r}\right)=D\left(\mathbb{C}, \mathbf{H}, \mathcal{H}^{r}, \dot{\mathcal{H}}^{r}\right)
$$

and ensures a symmetric, butterfly-type magnetostriction response for the $h$-MRE (as discuss in Section 6).

Material frame indifference. This physical property imposes that $W$ and $D$ must remain invariant under a change of observer. A change of observer leads to the new current position vector $\mathbf{x}^{*}=\mathbf{c}+\mathbf{Q x}$, where $\mathbf{c}$ is a rigid displacement field and $\mathbf{Q}$ is a proper rotation matrix (Gurtin, 1982, p. 139-142). Since the arguments of $W$ and $D$, i.e. $\mathbb{C}, \mathbf{H}, \mathcal{H}^{r}$ and $\dot{\mathcal{H}}^{r}$ are unaffected by such a transform, the requirement of material frame indifference imposes no further restrictions on $W$ and $D$. This observation is in agreement with the objectivity conditions used in mechanical visco-plasticity, where the intermediate strain-like variables remain unaffected by a change of observer (Dashner, 1993; Kumar and Lopez-Pamies, 2016).

Material symmetry. For isotropic MREs, $W$ and $D$ must remain invariant under a change in the reference configuration via a constant matrix $\mathbf{K} \in O r t h^{+}$. The material symmetry conditions on the potentials thus read

$$
\begin{gathered}
W\left(\mathbf{K}^{T} \mathbb{C} \mathbf{K}, \mathbf{K}^{T} \mathbf{H}, \mathbf{K}^{T} \mathcal{H}^{r}\right)=W\left(\mathbb{C}, \mathbf{H}, \mathcal{H}^{r}\right), \\
D\left(\mathbf{K}^{T} \mathbb{C} \mathbf{K}, \mathbf{K}^{T} \mathbf{H}, \mathbf{K}^{T} \mathcal{H}^{r}, \mathbf{K}^{T} \dot{\mathcal{H}}^{r}\right)=D\left(\mathbb{C}, \mathbf{H}, \mathcal{H}^{r}, \dot{\mathcal{H}}^{r}\right) .
\end{gathered}
$$

Here, we note that the intermediate $\mathcal{H}^{r}$ transforms as $\mathcal{H}^{r} \rightarrow \mathbf{K}^{T} \boldsymbol{H}^{r}$, which follows from (3.1) and the transformation $\mathbf{U} \rightarrow \mathbf{K}^{T} \mathbf{U K}$ under a change in the reference configuration. We further remark that in mechanical visco-plasticity the change in the reference configuration also modifies the intermediate plastic internal variables (Dashner, 1993; Bennett et al., 2016).

\footnotetext{
${ }^{4}$ It is emphasized that $\mathbf{R}(\mathbf{F})=\mathbf{F}\left(\mathbf{F}^{T} \mathbf{F}\right)^{-1 / 2}$ and hence, the evaluation of the derivative $\partial \mathbf{R} / \partial \mathbf{F}$ is a non-trivial operation. In this regard, we use the explicit expression of this fourth order tensor, provided by Chen and Wheeler (1993).

${ }^{5}$ Here, $\boldsymbol{\sigma}^{\text {mech }}$ is merely a notation introduced in the literature and might appear to be ambiguous, since a solid that is pre-magnetized, i.e., with remanent magnetization, still creates equilibrated magnetic fields around it. Upon application of a mechanical load the corresponding magnetization and magnetic fields will evidently change as we will see in this work.
} 
Entropy imbalance. The dissipation inequality $(3.6)_{1}$ imposes an additional constraint on $D$

$$
\frac{\partial D}{\partial \dot{\mathcal{H}}^{r}}\left(\mathbb{C}, \mathbf{H}, \mathcal{H}^{r}, \dot{\mathcal{H}}^{r}\right) \cdot \dot{\mathcal{H}}^{r} \geq 0
$$

which is clearly satisfied if $D\left(\mathbb{C}, \mathbf{H}, \mathcal{H}^{r}, \dot{\mathcal{H}}^{r}\right)$ is a convex function of $\dot{\mathcal{H}}^{r}$.

Definition of invariants. In order to satisfy the conditions of even magneto-mechanical coupling, isotropic material symmetry and frame indifference, we express the energy density function $W$ in terms of properly chosen isotropic invariants. In the present work, we consider the purely mechanical invariants

$$
I_{1}=\operatorname{tr}(\mathbb{C}), \quad I_{2}=\operatorname{tr}(\mathbb{C})^{2}-\operatorname{tr}\left(\mathbb{C}^{2}\right), \quad I_{3}=J^{2}=\operatorname{det} \mathbb{C},
$$

the uncoupled magnetic invariants

$$
I_{5}=\mathbf{h} \cdot \mathbf{h}=\mathbf{H} \cdot \mathbb{C}^{-1} \mathbf{H}, \quad I_{5}^{e r}=\mathbf{h} \cdot \mathbf{h}^{r}=\mathbf{H} \cdot \mathbb{C}^{-1 / 2} \mathcal{H}^{r}, \quad I_{5}^{r}=\mathbf{h}^{r} \cdot \mathbf{h}^{r}=\mathcal{H}^{r} \cdot \mathcal{H}^{r},
$$

and the coupled magneto-mechanical invariants

$$
I_{4}=\mathbf{h} \cdot \mathbb{B} \mathbf{h}=\mathbf{H} \cdot \mathbf{H}, \quad I_{4}^{e r}=\mathbf{h} \cdot \mathbb{B} \mathbf{h}^{r}=\mathbf{H} \cdot \mathbb{C}^{1 / 2} \mathcal{H}^{r}, \quad I_{4}^{r}=\mathbf{h}^{r} \cdot \mathbb{B} \mathbf{h}^{r}=\mathcal{H}^{r} \cdot \mathbb{C} \mathcal{H}^{r} .
$$

All the mechanical invariants along with $I_{4}$ and $I_{5}$ are the standard ones that are employed in non-dissipative magnetoelasticity (Keip and Rambausek, 2016, 2017; Lefèvre et al., 2017; Lefèvre et al., 2019; Mukherjee et al., 2020). We further note that the "uncoupled" $I_{5}$-type invariants are unaffected by mechanical deformation when a certain Eulerian magnetic field is independently controlled whereas the $I_{4}$ do vary in this case (see relevant discussion in Danas (2017)). Finally, in order to satisfy the invariance of the dissipation potential, we additionally employ the Euclidean norm $\left|\dot{\mathcal{H}}^{r}\right|=\sqrt{\dot{\mathcal{H}}^{r} \cdot \dot{\mathcal{H}}^{r}}$ as an invariant of $\dot{\mathcal{H}}^{r}$.

\section{Explicit definitions for the free energy density and the dissipation potential}

In this section, we propose explicit, analytical expressions for the free energy density $W$ and the dissipation potential $D$ using the previously defined invariants in the case of incompressible $h$-MREs. One of the main characteristics of the "microstructurally-guided" constitutive models presented here is that the macroscopic models almost exclusively depend on the properties of the underlying microstructure. Only a handful (usually one or two) additional parameters are introduced to allow for further calibration of the model.

For a better understanding, in the following, we first discuss the individual contributions to $W$ and $D$ step-by-step before specifying the evolution equations and an equivalent incremental variational formulation.

\subsection{The free energy density}

Following the guidelines of the work of Mukherjee et al. (2020) for $s$-MREs, we consider a free energy density for incompressible $h$-MREs in terms of the aforementioned invariants, so that

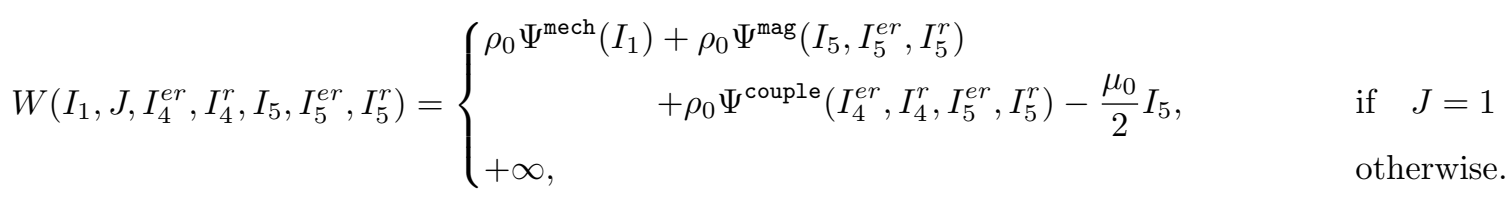

Here, $\Psi^{\text {mech }}$ and $\Psi^{\text {mag }}$ deliver a purely mechanical and purely magnetic response, respectively. In turn, $\Psi^{\text {couple }}$ describes the coupled magneto-mechanical response of the material. Finally, the last term $-\frac{\mu_{0}}{2} I_{5}$ takes into account the magnetic background (vacuum) energy.

The mechanical contribution $\Psi^{\text {mech }}$. For the effective mechanical energy we employ the analytical homogenization energy density of Lopez-Pamies et al. (2013) which is valid for general $I_{1}$-based, incompressible, hyperelastic composites comprising mechanically rigid particles, i.e.,

$$
\Psi^{\mathrm{mech}}\left(I_{1}\right)=(1-c) \Psi_{\mathrm{m}}^{\mathrm{mech}}\left(\mathcal{I}_{1}\right), \quad \mathcal{I}_{1}=\frac{I_{1}-3}{(1-c)^{7 / 2}}+3 .
$$

In this expression, $c$ is the volume fraction of the particles in the two-phase $h$-MRE composite (see for instance Fig. 3c) and $\Psi_{\mathrm{m}}^{\mathrm{mech}}$ represents the $I_{1}$-based isochoric mechanical free energy of the incompressible matrix (m) phase. A specific choice for $\Psi_{\mathrm{m}}^{\text {mech }}$ will be made at a later stage. It is interesting to note that $\Psi^{\text {mech }} \rightarrow+\infty$ as $c \rightarrow 1$, as a consequence of the rigid particles considered in this model.

Note further that for incompressible materials, the constitutive equation $(3.5)_{1}$ for the stress becomes

$$
\mathbf{S}=2 \mathbf{F} \frac{\partial W}{\partial \mathbb{C}}+p \mathbf{F}^{-T}
$$

where the pressure $p$ serves as the (negative) Lagrange multiplier associated with the incompressibility constraint $J=1$. 
The magnetic contribution $\Psi^{\mathrm{mag}}$. The purely magnetic energy is further decomposed into an energetic and a remanent part, so that

$$
\Psi^{\mathrm{mag}}\left(I_{5}, I_{5}^{e r}, I_{5}^{r}\right)=\Psi^{e, \operatorname{mag}}\left(I_{5}\right)+\Psi^{r, \operatorname{mag}}\left(I_{5}^{e r}, I_{5}^{r}\right) .
$$

Since there is no explicit analytical homogenization model available for the remanent magnetization response, we propose a decoupled effective model that satisfies certain limiting conditions. Specifically, the initial slopes of the effective magnetization in the proposed model are derived from corresponding analytical homogenization estimates and depend explicitly on the particle volume fraction $c$ as well as on the particle magnetic properties described next.

For a better understanding, we first describe graphically the particle magnetic properties (corresponding to the limiting case $c=1$ ) using a representative unidirectional magnetization hysteretic response in Fig. 2 (see Mukherjee and Danas (2019) for more details). Specifically, in Fig. 2a, an ideal hysteresis response is described by setting $\chi_{\mathrm{p}}^{e}=0$, which corresponds to no initial magnetization before switching ${ }^{6}$. Subsequently, the initial slope of magnetization at the onset of switching is given in terms of the particle remanent susceptibility $\chi_{\mathrm{p}}^{r}$, whereas the saturation magnetization is indicated by $m_{\mathrm{p}}^{s}$. The coercive field $b_{\mathrm{p}}^{c}$ controls the point where the magnetic switching initiates and is discussed in detail in Section 4.2.
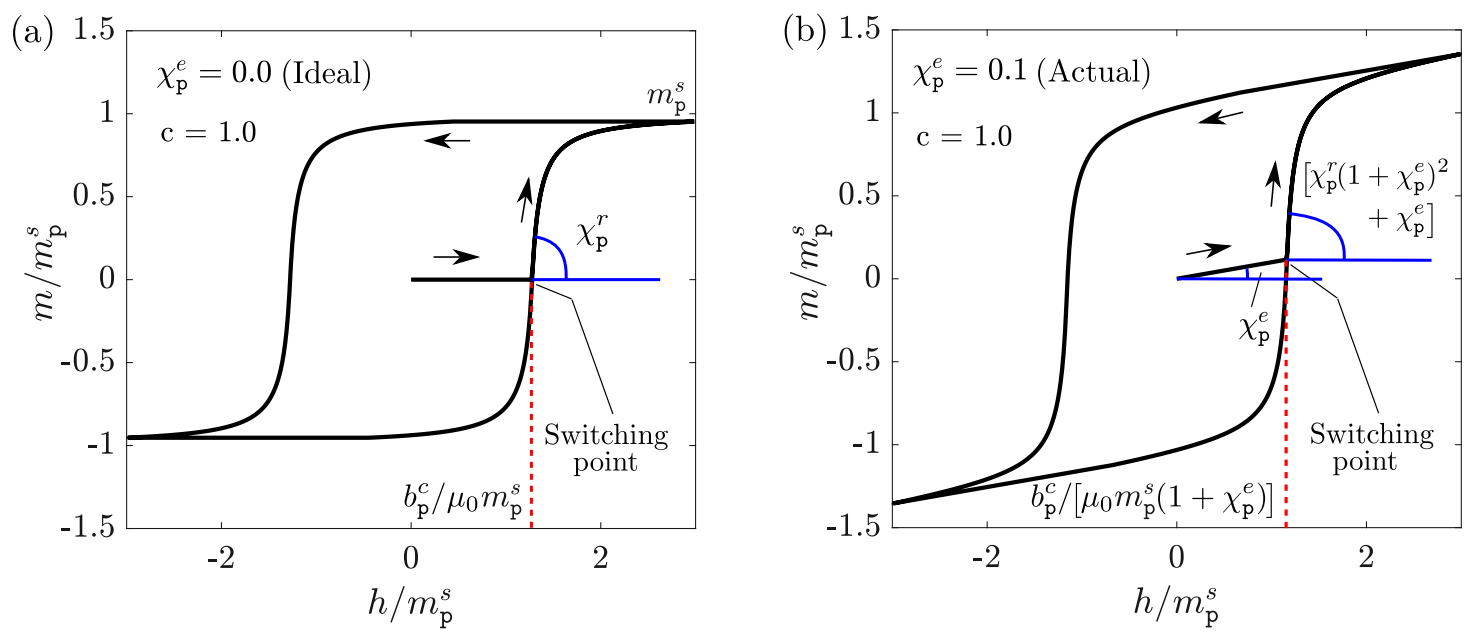

Figure 2: Representative magnetic hysteresis loops. (a) Ideal magnetic particles with no initial magnetization response $\left(\chi_{\mathrm{p}}^{e}=0\right)$, perfect saturation $m_{\mathrm{p}}^{s}$, coercive field $b_{\mathrm{p}}^{c}$ and the remanent susceptibility $\chi_{\mathrm{p}}^{r}$. (b) Actual hard magnetic particles (e.g. NdFeB) with an initial energetic magnetization $\left(\chi_{\mathrm{p}}^{e} \neq 0\right)$ which affects the unloading slope and a very slow saturating response and coercive field $b_{\mathrm{p}}^{c} /\left(1+\chi_{\mathrm{p}}^{e}\right)$. In both cases, the coercive field controls the point where the magnetic switching initiates and is normalized by $\mu_{0} m_{\mathrm{p}}^{s}$.

By contrast, as depicted in Fig. 2b, the actual hysteresis loops of hard magnets do not exhibit perfect saturation such that a certain slope of magnetization still remains even for high applied fields. This imperfect (or very slow) saturation is modeled in terms of the "energetic susceptibility" $\chi_{\mathrm{p}}^{e}$, which governs both the initial magnetization slope before switching as well as the magnetization slope at very high magnetic fields. The introduction of $\chi_{\mathrm{p}}^{e}$ thus modifies both the initial slope and the unloading slope of magnetization, as well as the ferromagnetic switching initiation controlled by the coercive field becoming $b_{\mathrm{p}}^{c} /\left[\mu_{0}\left(1+\chi_{\mathrm{p}}^{e}\right]\right.$ (see Fig. $\left.2 \mathrm{~b}\right)$. In turn, the corresponding slope at the onset of switching becomes $\chi_{\mathrm{p}}^{r}\left(1+\chi_{\mathrm{p}}^{e}\right)^{2}+\chi_{\mathrm{p}}^{e}$ (this result will become more clear in the following). For even more complex magnetic hysteretic responses, the reader is refereed to Mukherjee and Danas (2019).

In regard of this magnetic response of the particles, we propose, next, an effective magnetic free energy density for the $h$-MRE that ensures (i) the initial slopes of both, energetic and remanent magnetizations are given by the corresponding Maxwell-Garnett (M-G) lower bounds for the present case of two-phase, isotropic composites, (ii) the effective saturation magnetization for ideal magnets is given by $m^{s}=c m_{\mathrm{p}}^{s}$ and (iii) in the limiting case of only particles $(c=1)$, we recover the response in Fig. 2. The property (ii) reflects a homogenization result that holds true for $s$-MREs (see for instance Galipeau and Ponte Castañeda (2013) and Lefèvre et al. (2017)) as well as for $h$-MREs (as we will show in this study using numerical RVE simulations)

To achieve these properties, we first consider the energetic effective magnetic free energy to be a sum of an energetic and a remanent part, i.e.,

$$
\rho_{0} \Psi^{e, \operatorname{mag}}\left(I_{5}\right)=-\frac{\mu_{0}}{2} \chi^{e} I_{5}, \quad \chi^{e}=\frac{3 c \chi_{\mathrm{p}}^{e}}{3+(1-c) \chi_{\mathrm{p}}^{e}} .
$$

Here, $\chi^{e}$ is the effective Maxwell-Garnett lower bound for the energetic susceptibility of two-phase magnetic composites with one of the phases being non-magnetic (i.e. the matrix phase) given in terms of the particle volume fraction $c$ and the particle energetic susceptibility $\chi_{\mathrm{p}}^{e}$. This well-known homogenization result has been shown to deliver very accurate

\footnotetext{
${ }^{6}$ In Mukherjee and Danas (2019), the switching mechanism has been modeled by mimicking plastic yielding in an elasto-plastic problem.
} 
Table 1: Inverse saturation function choices for the remanent potential

\begin{tabular}{|l|c|c|}
\hline \multicolumn{1}{|c|}{ Function name } & $f_{\mathrm{i}}(\xi)$ & $f_{\dot{\mathrm{i}}}^{\prime}(\xi)$ \\
\hline Inverse hypergeometric function & $-[\log (1-\xi)+\xi]$ & $\frac{\xi}{1-\xi}$ \\
Tangent function & $-\frac{4}{\pi^{2}} \log \left[\cos \left(\frac{\pi}{2} \xi\right)\right]$ & $\frac{2}{\pi} \tan \left(\frac{\pi}{2} \xi\right)$ \\
Arctanh function & $-\left[(1-\xi) \tanh ^{-1}(\xi)-\log (\xi+1)\right]$ & $\tanh ^{-1}(\xi)$ \\
\hline
\end{tabular}

estimates when compared with corresponding experimental results in s-MREs (Psarra et al., 2017) and will also be shown to be the case in $h$-MREs by assessment with numerical RVE calculations in Section 6 .

Subsequently, the remanent magnetic free energy takes the form

$$
\rho_{0} \Psi^{r, \operatorname{mag}}\left(I_{5}^{e r}, I_{5}^{r}\right)=\mu_{0}\left(1+\chi^{e}\right) I_{5}^{e r}+\frac{\mu_{0}}{2}\left(\frac{1-c}{3 c}\right) I_{5}^{r}+\frac{\mu_{0}}{c} \frac{\left(m^{s}\right)^{2}}{\chi_{\mathrm{p}}^{r}} f_{\mathrm{p}}\left(\frac{\sqrt{I_{5}^{r}}}{m^{s}}\right), \quad m^{s}=c m_{\mathrm{p}}^{s}\left(\frac{1+\chi_{\mathrm{p}}^{e}}{1+\chi^{e}}\right)^{q_{\mathrm{sat}}} .
$$

In this expression, $q_{\text {sat }}=1$ is an exponent calibrated for fine-tunning the numerical RVE simulations in Section 6 and affects only weakly the effective saturation magnetization estimate of the actual magnets. It becomes inconsequential for ideal magnets, where $\chi^{e}=\chi_{\mathrm{p}}^{e}=0$. The general function $f_{\mathrm{p}}$ describes the saturation response of the material and is such that $f_{\mathrm{p}}^{\prime}$ becomes a inverse sigmoid function, which approaches $+\infty$ when its argument tends to 1 . Examples of such functions are provided in Table 1. In the present work, we employ the first choice, i.e., the inverse hypergeometric function $f_{\mathrm{p}}(\xi)=-[\log (1-\xi)+\xi]$.

One can show via straightforward algebraic manipulations that by considering the series expansion for small $\mathcal{H}^{r}$ in (4.6) and by retaining linear and quadratic terms with respect $\mathcal{H}^{r}, \Psi^{r \text {,mag }}$ becomes

$$
\rho_{0} \Psi^{r, \operatorname{mag}}\left(I_{5}^{e r}, I_{5}^{r}\right)=\mu_{0}\left(1+\chi^{e}\right) I_{5}^{e r}+\frac{\mu_{0}}{2 \chi^{r}} I_{5}^{r}+\mathcal{O}\left(\left|\mathcal{H}^{r}\right|^{3}\right), \quad \chi^{r}=\frac{3 c \chi_{\mathrm{p}}^{r}}{3+(1-c) \chi_{\mathrm{p}}^{r}} .
$$

In equation (4.7), $\chi^{r}$ corresponds to the Maxwell-Garnett lower bound for two-phase magnetic composites with one of the phases being non-magnetic and the other being the particle described by a remanent susceptibility $\chi_{\mathrm{p}}^{r}$. This last energy (4.7) leads to an effective initial slope at the onset of switching equal to $\chi^{r}$ and $\chi^{r}\left(1+\chi^{e}\right)^{2}+\chi^{e}$ for $h$-MREs comprising ideal and actual magnetic particles, respectively, in the limit of $\mathbb{C}=\mathbf{I}$. These slopes may be interpreted in a similar fashion to those for the pure particles $(c=1)$, shown in Fig. 2.

Furthermore, the limiting case of hard magnetic particles only, whose entire hysteretic response is described in Fig. 2, is readily obtained by setting $c=1$ in (4.4),(4.5), (4.6) and (4.7) leading to $\chi^{e}=\chi_{\mathrm{p}}^{e}, \chi^{r}=\chi_{\mathrm{p}}^{r}$ and $m^{s}=m_{\mathrm{p}}^{s}$ in that limit.

The coupling energy density $\Psi^{\text {couple }}$. Finally, the coupling free energy density $\rho_{0} \Psi_{\text {couple }}$ is assumed to take the form

$$
\rho_{0} \Psi^{\text {couple }}\left(I_{4}^{r}, I_{4}^{e r}, I_{5}^{r}, I_{5}^{e r}\right)=c \beta(c) \mu_{0}\left[\left(I_{4}^{r}-I_{5}^{r}\right)-2 \chi^{e}\left(I_{4}^{e r}-I_{5}^{e r}\right)\right] .
$$

Here, $\beta(c)$ is an calibration function of the particle volume fraction $c$ that controls the amplitude of the hysteretic magnetostriction response. At this point we keep $\beta$ general, while a specific form is proposed and discussed in Section 6.1 when the model response is assessed with the full-field numerical homogenization estimates.

Remark 2. We note that quasi-incompressible models may often be more convenient in numerical implementations. Thus, we extend in an ad-hoc manner the previous model to quasi-incompressible $h$-MREs, such that

$$
W_{\text {comp }}\left(I_{1}, J, I_{4}^{r}, I_{4}^{e r}, I_{5}, I_{5}^{r}, I_{5}^{e r}\right)=\rho_{0} \Psi_{\text {comp }}^{\text {mech }}\left(I_{1}, J\right)+\rho_{0} \Psi^{\mathrm{mag}}\left(I_{5}, I_{5}^{r}, I_{5}^{e r}\right)+\rho_{0} \Psi^{\text {couple }}\left(I_{4}^{r}, I_{4}^{e r}, I_{5}^{r}, I_{5}^{e r}\right)-\frac{\mu_{0}}{2} J I_{5},
$$

where

$$
\rho_{0} \Psi_{\text {mech }}^{\text {comp }}\left(I_{1}, J\right)=(1-c) \rho_{0} \Psi_{\mathrm{m}}^{\mathrm{mech}}\left(\mathcal{I}_{1}^{\mathrm{comp}}\right)+\frac{G_{\mathrm{m}}^{\prime}}{2(1-c)^{6}}(J-1)^{2}, \quad \mathcal{I}_{1}^{\mathrm{comp}}=\frac{I_{1}-3-2 \ln J}{(1-c)^{7 / 2}}+3 .
$$

The Lamé parameter $G_{\mathrm{m}}^{\prime}$ penalizing volumetric deformation of the matrix phase is usually set to values $G_{\mathrm{m}}^{\prime} \geq 500 G_{\mathrm{m}}$ to ensure a good approximation for incompressibility. It should be noted that the last term in (4.10) has to be underintegrated in displacement-based finite element schemes in order to avoid volumetric locking. The proposed quasiincompressible model has been validated for large values of $G_{\mathrm{m}}^{\prime}$ and should be used with caution for lower ones. Specifically, if one deals with compressible $h$-MREs, coupling of $J$ with the magnetic fields is expected to play an important role and thus the present model may become inaccurate for such cases. 


\subsection{The dissipation potential}

It remains to define the evolution law for the internal variable $\mathcal{H}^{r}$, which is obtained in terms of a magnetic dissipation potential. A positive dissipation is guaranteed when this potential is (strictly) convex. We start by considering a ratedependent dissipation potential (similar to visco-plasticity and creep in mechanics) and subsequently consider the rate independent limit. Specifically, we write the simple power-law potential

$$
D\left(\dot{\mathcal{H}}^{r}\right)=\frac{n b^{c}}{n+1}\left|\dot{\mathcal{H}}^{r}\right|^{\frac{n+1}{n}}, \quad 1 \leq n \leq \infty .
$$

In this expression, $b^{c}$ denots the effective coercive field of the composite, $n$ is the rate exponent and $\left|\dot{\mathcal{H}}^{r}\right|=\sqrt{\dot{\mathcal{H}}^{r} \cdot \dot{\mathcal{H}}^{r}}$ denotes the Euclidean norm. A value of $n=1$ leads to a rate-dependent linear switching response (similar to linear viscoelasticity) and $n=\infty$ leads to a highly non-linear rate-independent response (similar to perfect plasticity) (Danas and Ponte Castañeda, 2009; Rosato and Miehe, 2014). The dissipation potential is strictly convex everywhere except when $n=\infty$ that becomes simply convex.

Our numerical homogenization estimates (see Section 6) and corresponding experiments (Huber et al., 2017; Kim et al., 2018) show that the effective coercivity $b^{c}$ of the composite is equal to the coercivity of the inclusions $b_{\mathrm{p}}^{c}$ for ideal magnets, whereas it exhibits a very weak dependence on $c$ for actual magnets. To capture both effects, we set

$$
b^{c}=b_{\mathrm{p}}^{c}\left(\frac{1+\chi^{e}}{1+\chi_{\mathrm{p}}^{e}}\right)^{q_{c o e r}},
$$

where $\chi^{e}$ is defined in (4.5), $\chi_{\mathrm{p}}^{e}$ is the energetic susceptibility of the particles and $q_{\mathrm{coer}}=4 / 5$ is a calibration exponent that becomes inconsequential for ideal magnets, where $\chi^{e}=\chi_{\mathrm{p}}^{e}=0$. We also remark that the dissipation potential in (4.11) is independent of mechanical stretch since $\dot{\mathcal{H}}^{r}$ is defined in the intermediate configuration.

Relation (3.7) 1 yields the intermediate remanent field $\mathcal{B}^{r}$ as the work conjugate of $\dot{\mathcal{H}}^{r}$. Thus, a Legendre transform of $D\left(\dot{\mathcal{H}}^{r}\right)$ with respect to $\dot{\mathcal{H}}^{r}$ leads to its complimentary dissipation potential $D^{*}\left(\mathcal{B}^{r}\right)=b^{c}\left(\left|\mathcal{B}^{r}\right| / b^{c}\right)^{n+1} /(n+1)$, which is also a power-law with an exponent $n+1$ on $\left|\mathcal{B}^{r}\right|=\sqrt{\mathcal{B}^{r} \cdot \mathcal{B}^{r}}$. Subsequently, in the limit of rate-independence, i.e., $n=\infty$, the dual dissipation potential $D^{*}$ becomes

$$
n=\infty \quad \Longrightarrow \quad D^{*}\left(\mathcal{B}^{r}\right)= \begin{cases}0, & \text { if } \quad\left|\mathcal{B}^{r}\right| \leq b^{c} \\ \infty, & \text { otherwise }\end{cases}
$$

This readily leads to the switching surface (similar to yield surface in plasticity) definition

$$
\Phi\left(\mathcal{B}^{r}\right):=\left|\mathcal{B}^{r}\right|^{2}-\left(b^{c}\right)^{2}=0 .
$$

Finally, the evolution equation for $\mathcal{H}^{r}$ is obtained from the principle of maximum remanent dissipation that leads to the associated switching (normality) rule in the intermediate configuration

$$
\dot{\mathcal{H}}^{r}=\dot{\Lambda} \frac{\partial \Phi}{\partial \mathcal{B}^{r}} \quad \text { or } \quad \Delta \mathcal{H}^{r}=\Delta \Lambda \frac{\partial \Phi}{\partial \mathcal{B}^{r}} .
$$

Here, $\Delta \Lambda$ is a Lagrange multiplier that satisfies the Kuhn-Tucker conditions

$$
\Delta \Lambda \geq 0, \quad \Phi \leq 0 \quad \text { and } \quad \Delta \Lambda \Phi=0
$$

In summary, the constitutive response of an $h$-MRE may be fully defined in terms of the the primary relations (3.5), the remanent constitutive relation (3.6) $)_{2}$ and the associated switching rule (4.15) along with the Kuhn-Tucker conditions (4.16). The numerical implementation of these constitutive equations is discussed in briefly in the next section, while a complete description is left for a separate communication which is in progress.

Remark 3. Following Mukherjee and Danas (2019), we note that the switching surface-based hard ferromagnetic hysteresis model yields the non-dissipative saturation-type magnetization response in the limit of $b_{\mathrm{p}}^{c} \rightarrow 0$ (along with $\chi_{\mathrm{p}}^{e}=0$ ), which imply that $b^{c} \rightarrow 0$ and $\chi^{e}=0$ for the $h$-MRE too. In those limits, the proposed macroscopic model for $h$-MREs recovers - upon proper calibration of the $\beta$ function - the corresponding homogenization model for $s$-MREs of(Lefèvre et al., 2017) and thus that of Mukherjee et al. (2020). The reader is referred to the thesis manuscript of Mukherjee (2020) for a more detailed analysis of this point.

\subsection{The limiting cases of pure particle and pure matrix}

We discuss, now, two important limiting cases corresponding to a pure magnetic particle response, obtained easily by setting $c=1$, and a pure non-magnetic matrix response, obtained by considering carefully the limit $c \rightarrow 0$, in the previous expressions. Those two limiting cases will be used in our numerical computations to analyze directly a RVE comprising particles embedded in a polymer matrix. Thus, one needs to be certain that the proposed $h$-MRE model recovers seamlessly those two limits. This, in turn, allows for a straightforward and unique numerical implementation of the proposed constitutive model. 
The limiting case of $c=1$. This case corresponds to the pure particle response, which is considered to be magnetic but mechanically rigid (i.e. $\mathbb{C}=\mathbf{I}$ in this case). In that case, the magnetic model simplifies further upon substitution of $c=1$ into (4.5) and (4.6). Moreover, the coupling free energy (4.8) vanishes, since all magneto-mechanical and magnetic invariants become identical for $\mathbb{C}=\mathbf{I}$. Thus, the energy density reads after some straightforward algebraic manipulations as

$$
W_{c \rightarrow 1}\left(I_{1}, I_{5}, I_{5}^{e r}, I_{5}^{r}\right)= \begin{cases}-\frac{\mu_{0}}{2}\left(1+\chi_{\mathrm{p}}^{e}\right) I_{5}+\mu_{0}\left(1+\chi_{\mathrm{p}}^{e}\right) I_{5}^{e r}+\mu_{0} \frac{\left(m_{\mathrm{p}}^{s}\right)^{2}}{\chi_{\mathrm{p}}^{r}} f_{\mathrm{p}}\left(\frac{\sqrt{I_{5}^{r}}}{m_{\mathrm{p}}^{s}}\right) & \text { if } \quad \mathbb{C}=\mathbf{I} \\ +\infty & \text { otherwise }\end{cases}
$$

The corresponding switching surface $\Phi$ in this limit reads

$$
\Phi_{c \rightarrow 1}\left(\mathcal{B}^{r}\right)=\left|\mathcal{B}^{r}\right|^{2}-\left(b_{\mathrm{p}}^{c}\right)^{2}
$$

The magnetization response obtained from the energy density (4.17) and switching surface (4.18) is shown in Fig. 2a and $2 \mathrm{~b}$, having $\chi_{\mathrm{p}}^{e}=0$ and 0.1 , respectively. In practice, one can easily obtain a similar numerical response by setting sufficiently large mechanical properties in (4.2) (e.g., the shear and bulk moduli) and $c=1$. This leads to $\rho_{0} \Psi^{\text {mech }}(\mathbb{C}) \rightarrow 0$ and $\mathbb{C} \rightarrow \mathbf{I}$, while the only non-zero energetic contributions come from the corresponding magnetic parts.

The limiting case of $c=0$. This case results in a mechanical free energy equal to that of the matrix phase, i.e. $\Psi_{\mathrm{m}}^{\mathrm{mech}}$. Furthermore, the coupling energy vanishes identically in (4.8) for $c=0$. In turn, the corresponding magnetic free energy $\Psi^{\mathrm{mag}}$ in (4.6) becomes for $c \rightarrow 0$ equal to

$$
\rho_{0} \Psi^{\mathrm{mag}}= \begin{cases}0, & \text { if } \boldsymbol{\mathcal { H }}^{r}=\mathbf{0} \\ +\infty, & \text { if } \quad \mathcal{H}^{r} \neq \mathbf{0}\end{cases}
$$

This last relation, thus, imposes the constraint $\mathcal{H}^{r}=\mathbf{0}$ when $c=0$ for the energy of the solid to remain bounded. As a result of (4.19), the energy density for the special case of $c=0$ is simply given by

$$
W_{c \rightarrow 0}\left(I_{1}, I_{5}\right)=\rho_{0} \Psi_{\mathrm{m}}^{\mathrm{mech}}\left(I_{1}\right)-\frac{\mu_{0}}{2} I_{5}
$$

for the incompressible case, which is the common energy density for non-magnetic solids (see for instance Mukherjee et al. (2020); Lefèvre et al. (2020)). Of course, the dissipation potential $D$ vanishes identically in this limit and the magnetic constitutive relation for non-magnetic media leads to $\mathbf{b}=\mu_{0} \mathbf{h}$ and thus to $\mathbf{m}=\mathbf{0}$ in the matrix.

\subsection{Equivalent incremental variational formulation}

The local constitutive equations proposed in the previous sections can be recast in an equivalent incremental variational form. Such an exercise is carried out as it will prove extremely useful in Section 5, where we discuss the numerical homogenization of $h$-MRE RVEs. The idea of such an incremental potential is borrowed from existing literature on dissipative media (Ortiz and Stanier, 1999; Miehe, 2002; Miehe et al., 2002; Hackl and Fischer, 2007; Miehe et al., 2011) and is appropriately adapted in the present context of $h$-MREs.

Variational principles for dissipative solids are constructed for the time interval associated with a time increment $\Delta t$, i.e. $[t, t+\Delta t]$ such that the corresponding Euler-Lagrange equations yield both the balance laws for the primary fields and the evolution laws corresponding to the dissipative processes. For simplicity in notation, we henceforth replace $t+\Delta t$ by the symbol " $\tau$ ".

In this work, one can devise an incremental potential $\mathcal{W}$ that accounts for the evolution of internal variables of the form

$$
\mathcal{W}\left(\mathbf{F}_{\tau}, \mathbf{H}_{\tau}\right)=\inf _{\mathcal{H}_{\tau}^{r}}\left[\int_{t}^{\tau}\left\{\dot{W}\left(\mathbf{F}, \mathbf{H}, \mathcal{H}^{r}\right)+D\left(\dot{\mathcal{H}}^{r}\right) \mathrm{d} t\right\}\right] \quad \text { with } \quad \mathcal{H}^{r}(t)=\mathcal{H}_{t}^{r}
$$

being the initial value of $\mathcal{H}^{r}$ in the time increment $[t, \tau]$. In $(4.21)$ the potentials $W\left(\mathbb{C}, \mathbf{H}, \mathcal{H}^{r}\right)$ and $D\left(\dot{\mathcal{H}}^{r}\right)$ are given by (4.9) and (4.11), respectively. Considering now the Legendre-Fenchel transform of $D=\sup _{\mathcal{B}^{r}}\left(\mathcal{B}^{r} \cdot \dot{\mathcal{H}}^{r}-D^{*}\left(\mathcal{B}^{r}\right)\right)$ together with the definition of $D^{\mathrm{H} *}\left(\boldsymbol{B}^{r}\right)$ in the rate-independent limit $n=\infty$, we obtain the constrained optimization problem

$$
\mathcal{W}\left(\mathbf{F}_{\tau}, \mathbf{H}_{\tau}\right)=\inf _{\mathcal{H}_{\tau}^{r}} \sup _{\mathcal{B}_{\tau}^{r} \in \mathcal{B}}\left[W\left(\mathbf{F}_{\tau}, \mathbf{H}_{\tau}, \mathcal{H}_{\tau}^{r}\right)-W\left(\mathbf{F}_{t}, \mathbf{H}_{t}, \mathcal{H}_{t}^{r}\right)+\mathcal{B}_{\tau}^{r} \cdot\left\{\mathcal{H}_{\tau}^{r}-\mathcal{H}_{t}^{r}\right\}\right],
$$

with

$$
\mathcal{B}=\left\{\mathcal{B}^{r}: \Phi\left(\left|\mathcal{B}^{r}\right|\right)=\left|\mathcal{B}^{r}\right|^{2}-\left(b^{c}\right)^{2} \leq 0\right\}
$$


The inequality constraint in (4.23) can be imposed on (4.22) with the aid of a Lagrange multiplier, $\Delta \Lambda \geq 0$, such that

$$
\mathcal{W}\left(\mathbf{F}_{\tau}, \mathbf{H}_{\tau}\right)=\inf _{\mathcal{H}_{\tau}^{r}} \sup _{\mathcal{B}_{\tau}^{r}} \inf _{\Delta \Lambda \geq 0}\left[W\left(\mathbf{F}_{\tau}, \mathbf{H}_{\tau}, \mathcal{H}_{\tau}^{r}\right)-W\left(\mathbf{F}_{t}, \mathbf{H}_{t}, \mathcal{H}_{t}^{r}\right)+\mathcal{B}_{\tau}^{r} \cdot\left\{\mathcal{H}_{\tau}^{r}-\mathcal{H}_{t}^{r}\right\}-\Delta \Lambda \Phi\left(\boldsymbol{B}_{\tau}^{r}\right)\right]
$$

This last optimization problem leads readily to time discrete forms of the constitutive relation (3.6) ${ }_{2}$ and the evolution of the internal state (4.15) respecting the Kuhn-Tucker conditions (4.16).

In the present study, we obtain the internal state $\mathcal{H}_{\tau}^{r}$ and the auxiliary $\mathcal{B}_{\tau}^{r}$ and $\Delta \Lambda$ by solving the optimization problem in (4.24) with a standard Newton-Raphson scheme. An alternative and perhaps more efficient implementation can be based on a radial-return-type approach (Wilkins, 1964). The reader is referred to the Appendix A of (Mukherjee and Danas, 2019) or Chapter 4 of (Mukherjee, 2020) for additional details on the update algorithm. The updated $\mathcal{H}_{\tau}^{r}, \boldsymbol{B}_{\tau}^{r}$ and $\Delta \Lambda$ are then substituted in (4.24) to obtain the optimized incremental potential for given values of the deformation gradient $\mathbf{F}$ and magnetic h-field $\mathbf{H}$. The incremental constitutive relations are then obtained by substituting (4.24) into (3.5), such that

$$
\mathbf{S}_{\tau}=\frac{\partial \mathcal{W}}{\partial \mathbf{F}_{\tau}}\left(\mathbf{F}_{\tau}, \mathbf{H}_{\tau}\right), \quad \mathbf{B}_{\tau}=-\frac{\partial \mathcal{W}}{\partial \mathbf{H}_{\tau}}\left(\mathbf{F}_{\tau}, \mathbf{H}_{\tau}\right)
$$

\section{Effective incremental response: numerical homogenization}

This section describes the numerical homogenization framework used to analyze three-dimensional representative volume elements (RVEs) of $h$-MREs. Those computations, besides allowing for an in-depth understanding of the microdeformation mechanisms leading to the overall magneto-mechanical coupling, serve also to assess the accuracy of the previously-proposed explicit macroscopic model at different particle volume fractions and a variety of loading conditions.

(a) Boundary value problem

(b) Periodic RVE

(c) RVE
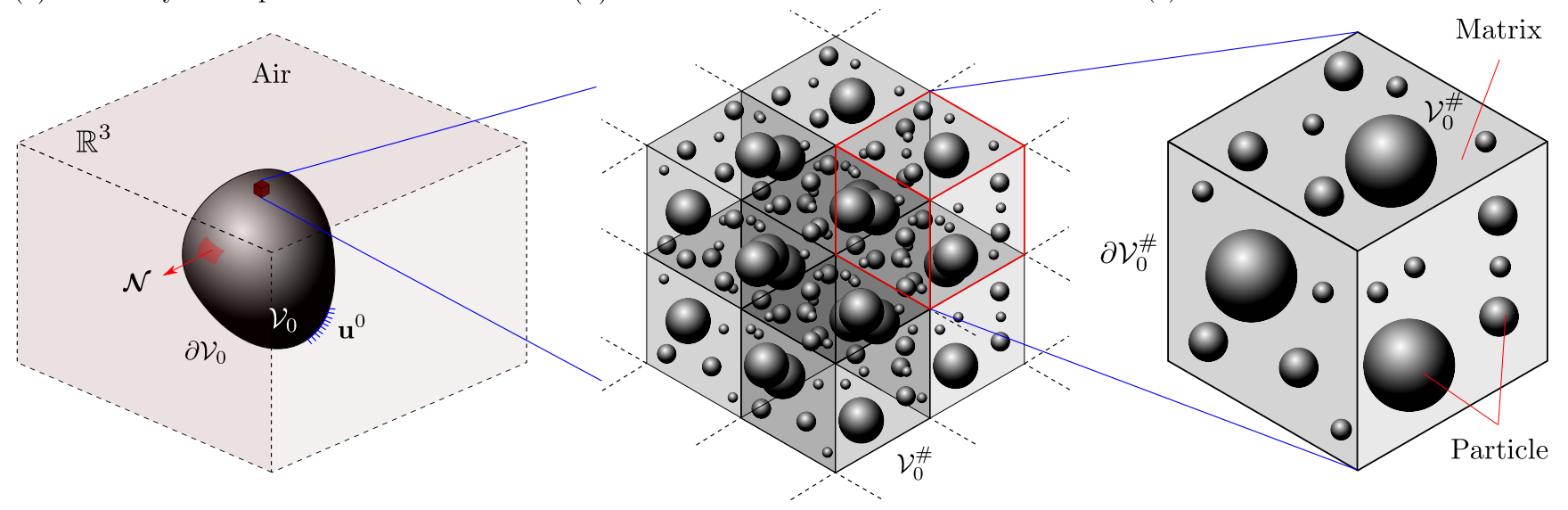

Figure 3: Schematic diagram of (a) macroscopic boundary value problem involving a MRE sample in air having a reference volume $\mathcal{V}_{0}$ with unit normal $\mathcal{N}$ on the boundary $\partial \mathcal{V}_{0}$ and a representative boundary with fixed displacement $\mathbf{u}_{0}$, (b) periodic arrangement of a RVE with polydisperse spherical inclusions and (c) a RVE occupying a reference volume $\mathcal{V}_{0}^{\#}$ and boundary $\partial \mathcal{V}_{0}^{\#}$.

As shown in Fig. 3, each point of the macro-continuum $\mathcal{V}_{0}$ (Fig. 3a) is assumed to be described well at the microscale by a representative volume element (RVE) having a reference volume of $\mathcal{V}_{0}^{\#}$ and comprising two phases, denoted as $i=p, m$ representing the particle and matrix phases, respectively (Fig. 3c). This assumption may be considered sufficient for the present $h$-MRE composites provided that the particle size is sufficiently smaller than the specimen analyzed ${ }^{7}$. Then, for slowly varying mechanical and magnetic fields at the macroscopic scales, the previous microstructural assumptions allow for separation of length scales $\left(\mathcal{V}_{0}^{\#} \ll \mathcal{V}_{0}\right)$. In addition, following Danas (2017), we consider a slowly varying microstructure, so that the microstructure can be assumed to be (locally) periodic (see Fig. 3b). This interpretation results in periodic boundary conditions applied on a single RVE (see Fig. 3b).

\subsection{Local energy density of the constituents}

Henceforth, the microscopic field variables along with the energy functions and the corresponding invariants are indicated with a $(\cdot)$ symbol in order to distinguish them from their macroscopic counterparts. Since the microstructure

\footnotetext{
${ }^{7}$ The particle size in typical $h$-MREs is in the order of $10-30 \mu \mathrm{m}$, while a cubic RVE as we will see contains approximately five particles per direction, i.e., has a side of $\sim 50-150 \mu \mathrm{m}$ at moderate volume fractions. The specimen sizes in actual experiments are usually in the centimeter scale and thus are sufficiently larger than the microstructure. In turn, such models including any type of phenomenological ones should be used with caution in the context of slender structures such as those in Psarra et al. (2017) and Kim et al. (2018), where one or two of the specimen dimensions may be a fraction of a millimeter. In that case, additional calibration may be required.
} 
is heterogeneous, the referential representations of the local energy density $\breve{W}$ and the local dissipation potential $\breve{D}$ both depend on the reference coordinate $\mathbf{X}$, so that

$$
\widetilde{W}\left(\mathbf{X}, \check{\mathbb{C}}, \check{\mathbf{H}}, \breve{\mathcal{H}}^{r}\right)=\Theta(\mathbf{X}) \breve{W}_{\mathrm{m}}\left(\check{\mathbb{C}}, \check{\mathbf{H}}, \check{\mathcal{H}}^{r}\right)+(1-\Theta(\mathbf{X})) \widetilde{W_{\mathrm{p}}}\left(\check{\mathbb{C}}, \check{\mathbf{H}}, \breve{\mathcal{H}}^{r}\right)
$$

and

$$
\check{D}\left(\mathbf{X}, \dot{\breve{\mathcal{H}}}^{r}\right)=\Theta(\mathbf{X}) \breve{D}_{\mathrm{m}}\left(\dot{\overline{\mathcal{H}}}^{r}\right)+(1-\Theta(\mathbf{X})) \breve{D}_{\mathrm{p}}\left(\dot{\overrightarrow{\mathcal{H}}}^{r}\right)
$$

respectively. In this last two expressions, $\Theta(\mathbf{X})$ denotes the characteristic function taking the value $\Theta(\mathbf{X})=1$ if $\mathbf{X} \in \mathcal{V}_{0}^{\# \mathrm{~m}}$ and $\Theta(\mathbf{X})=0$ if $\mathbf{X} \in \mathcal{V}_{0}^{\# \mathrm{p}}$. The microscopic energy densities $\widetilde{W}_{\mathrm{p}}$ and $\widetilde{W}_{\mathrm{m}}$ are given by (4.17) and (4.20), respectively, i.e., the pure particle $(c=1)$ and pure matrix $(c \rightarrow 0)$ limiting cases. Of course, now all the field variables in (4.17) and (4.20) must be replaced with the corresponding overscript ones $\widetilde{(\cdot)}$. Further note that (4.9) already features the quasi-incompressible mechanical response which is more convenient in numerical simulations. Finally, in (5.2), we may readily set $\breve{D}_{\mathrm{m}}=0$ since the magnetic dissipation is identically zero in the non-magnetic polymer matrix.

\subsection{Incremental homogenization framework}

This section discusses briefly the incremental periodic homogenization framework for $h$-MREs based on an incremental micro-potential $\widetilde{\mathcal{W}}\left(\mathbf{X}, \breve{\mathbf{F}}_{\tau}, \breve{\mathbf{H}}_{\tau}\right)$, which can be defined by substituting all quantities in (4.21) with an overscript $\widetilde{(\cdot)}$. The average deformation gradient $\mathbf{F}$ and Lagrangian $h$-field $\mathbf{H}$ at a discrete time $\tau \equiv t+\Delta t$ are then expressed in terms of the volume averages of the corresponding microscopic quantities, so that

$$
\mathbf{F}_{\tau}=\frac{1}{\left|\mathcal{V}_{0}^{\#}\right|} \int_{\mathcal{V}_{0}^{\#}} \check{\mathbf{F}}_{\tau}(\mathbf{X}) \mathrm{d} V, \quad \mathbf{H}_{\tau}=\frac{1}{\left|\mathcal{V}_{0}^{\#}\right|} \int_{\mathcal{V}_{0}^{\#}} \check{\mathbf{H}}_{\tau}(\mathbf{X}) \mathrm{d} V
$$

respectively. The microscopic displacements $\breve{\mathbf{u}}_{\tau}(\mathbf{X})$ and the microscopic scalar potential $\breve{\varphi}_{\tau}(\mathbf{X})$ are additively decomposed into linear (macroscopic) and higher order (microscopic fluctuation) contributions ${ }^{8}$

$$
\check{\mathbf{u}}_{\tau}(\mathbf{X})=\left(\mathbf{F}_{\tau}-\mathbf{I}\right) \cdot \mathbf{X}+\widetilde{\mathbf{u}}_{\tau}(\mathbf{X}) \quad \text { and } \quad \check{\varphi}_{\tau}(\mathbf{X})=-\mathbf{H}_{\tau} \cdot \mathbf{X}+\widetilde{\varphi}_{\tau}(\mathbf{X}), \quad \forall \mathbf{X} \in \mathcal{V}_{0}^{\#},
$$

where $\widetilde{\mathbf{u}}_{\tau}(\mathbf{X})$ and $\widetilde{\varphi}_{\tau}(\mathbf{X})$ are the fluctuation fields. Their average over $\mathcal{V}_{0}^{\#}$ is required to vanish such that (5.4) is consistent with (5.3), which is automatically fulfilled for $\mathcal{V}_{0}^{\#}$-periodic fluctuation fields.

Based on the above definitions, the incremental homogenized potential for the numerical RVE reads

$$
\mathcal{W}\left(\mathbf{F}_{\tau}, \mathbf{H}_{\tau}\right)=\inf _{\check{\mathbf{u}}_{\tau} \in \mathcal{K}\left(\mathbf{F}_{\tau}\right)} \sup _{\check{\varphi}_{\tau} \in \mathcal{G}\left(\mathbf{H}_{\tau}\right)}\left[\frac{1}{\mathcal{V}_{0}^{\#}} \int_{\mathcal{V}_{0}^{\#}} \widetilde{\mathcal{W}}\left(\mathbf{X}, \check{\mathbf{F}}_{\tau}, \check{\mathbf{H}}_{\tau}\right) \mathrm{d} V\right],
$$

where $\mathcal{K}$ and $\mathcal{G}$ represents the sets of admissible microscopic $\breve{\mathbf{u}}_{\tau}$ and $\breve{\varphi}_{\tau}$ fields, defined, respectively, as

$$
\mathcal{K}\left(\mathbf{F}_{\tau}\right)=\left\{\check{\mathbf{F}}_{\tau}=\mathbf{I}+\operatorname{Grad} \check{\mathbf{u}}_{\tau}, \breve{J}_{\tau}>0, \breve{\mathbf{u}}_{\tau}=\left(\mathbf{F}_{\tau}-\mathbf{I}\right) \cdot \mathbf{X}+\widetilde{\mathbf{u}}_{\tau}, \widetilde{\mathbf{u}}_{\tau} \text { periodic in } \mathcal{V}_{0}^{\#}\right\}
$$

and

$$
\mathcal{G}\left(\mathbf{H}_{\tau}\right)=\left\{\check{\mathbf{H}}_{\tau}=-\operatorname{Grad} \check{\varphi}_{\tau}, \check{\varphi}_{\tau}=-\mathbf{H}_{\tau} \cdot \mathbf{X}+\widetilde{\varphi}_{\tau}, \widetilde{\varphi}_{\tau} \text { periodic in } \mathcal{V}_{0}^{\#}\right\} .
$$

Applying then the Hill-Mandel lemma, we recover the macroscopic constitutive relations defined in (4.25).

At this stage, the definition of the incremental homogenization problem for $h$-MREs is formally complete and one could proceed to compare the explicit model with the numerical RVE homogenized response (5.5). Nevertheless, it has been shown in (Danas, 2017) that such use of (5.5) does not reveal properly the effective magneto-mechanical response that arises from interactions between the magnetic particles. Instead, further modifications to the incremental variational principle are necessary, as detailed in the following.

\subsection{Augmented macroscopic potential energy for RVE evaluations}

Recent works of Keip and Rambausek (2016), Danas (2017) and Mukherjee et al. (2020) pointed out a key difference between the electro-active and magneto-active boundary value problems. Electro-active elastomers are typically loaded by electrodes that are directly attached to the material. In contrast, the MREs are usually immersed in the magnetic field created by fixed poles of electromagnets that rest at a certain distance away from the MRE sample (Bodelot et al., 2017; Zhao et al., 2019). One of the main differences between those two problems is that in the first the electric fields are zero outside the body, implying a zero Maxwell stress in vacuum, while in the second the magnetic fields and thus the

\footnotetext{
${ }^{8}$ Notice that the deformation gradient $\mathbf{F}$ can be expressed in terms of the displacement gradient, so that $\mathbf{F}=\mathbf{I}+$ Gradu. The magnetic $\mathbf{H}$ field, by virtue of its curl-free property, can be expressed as $\mathbf{H}=-$ Grad $\varphi$, where $\varphi$ is a scalar potential. The last two relations are scale-independent and thus, remain equally valid for the macro and the micro scales.
} 
Maxwell stress are not zero. In an effort to appropriately take into account the pure magneto-mechanical coupling in the RVE, free from the effect of those macroscopic boundary conditions, Danas (2017) and Mukherjee et al. (2020) proposed an augmented potential energy that involves three additional loading terms to deal with the surrounding RVE medium, the applied Eulerian magnetic field and the potential control of an average mechanical stress field. This potential energy allows to describe properly the magnetic effects (including the Maxwell stresses) exerted by the surrounding RVEs on the RVE under study and is briefly revisited here for completeness. The reader is referred to (Danas, 2017) for a complete discussion on this highly non-trivial matter.

Specifically, the first additional term serves to describe the application of the current macroscopic $h$-field, $\mathbf{h}^{\text {app }}$, at the level of the RVE, instead of the referential one, $\mathbf{H}$. This may be achieved by the use of a penalty term $\frac{\mu_{0}}{2 \zeta}\left|\mathbf{F}_{\tau}^{-T} \mathbf{H}_{\tau}-\mathbf{h}_{\tau}^{\text {app }}\right|^{2}$ with $\zeta \ll 1$. Next, the macroscopic background energy $-\mu_{0} I_{5} / 2$ (or $-\mu_{0} J_{5} / 2$ in the quasi-incompressible case) is subtracted from (5.5). This accounts for the presence of the neighboring RVEs (see Fig. 3b) by imposing the continuity of the macroscopic Maxwell stresses between neighboring RVEs, far from the boundaries of the specimen. Finally, to be able to prescribe macroscopic mechanical stress $\mathbf{S}_{\tau}^{\text {mech }}$ instead of deformation $\mathbf{F}$, one may consider the term $\mathbf{S}_{\tau}^{\text {mech }}:\left(\mathbf{F}_{\tau}-\mathbf{I}\right)$. Assembling these three additional terms together, we obtain the augmented potential energy (Danas, 2017; Mukherjee et al., 2020)

$$
\mathscr{P}\left(\mathbf{F}_{\tau}, \mathbf{H}_{\tau}\right)=\mathcal{W}\left(\mathbf{F}_{\tau}, \mathbf{H}_{\tau}\right)+\frac{\mu_{0}}{2} \mathbf{F}_{\tau}^{-T} \mathbf{H}_{\tau} \cdot \mathbf{F}_{\tau}^{-T} \mathbf{H}_{\tau}+\frac{\mu_{0}}{2 \zeta}\left|\mathbf{F}_{\tau}^{-T} \mathbf{H}_{\tau}-\mathbf{h}_{\tau}^{\mathrm{app}}\right|^{2}-\mathbf{S}_{\tau}^{\mathrm{mech}}:\left(\mathbf{F}_{\tau}-\mathbf{I}\right),
$$

with $\mathcal{W}\left(\mathbf{F}_{\tau}, \mathbf{H}_{\tau}\right)$ defined by (4.24) for the analytical model and by (5.5) for the numerical RVE. The resulting EulerLagrange equations of the RVE response under the prescribed magnetic and mechanical loads introduced in (5.8) are obtained by setting $\delta \mathscr{P}\left(\mathbf{F}_{\tau}, \mathbf{H}_{\tau}\right)=0$, which leads to

$$
\mathbf{S}_{\tau}-\mathbf{S}_{\tau}^{\text {maxw }}-\mathbf{S}_{\tau}^{\text {mech }}=\mathbf{0}, \quad \mathbf{B}_{\tau}-\mu_{0} \mathbb{C}^{-1} \mathbf{H}_{\tau}-\frac{\mu_{0}}{\zeta} \mathbf{F}_{\tau}^{-1}\left(\mathbf{F}_{\tau}^{-T} \mathbf{H}_{\tau}-\mathbf{h}_{\tau}^{\text {app }}\right)=\mathbf{0}
$$

Here, $\mathbf{S}^{\text {maxw }}=J \boldsymbol{\sigma}^{\text {maxw}} \mathbf{F}^{-T}$ is the 1st Piola-Kirchhoff expression for the energetic Maxwell stress, whose expression in terms of $\mathbf{h}$ and $\mathbf{b}$ is given in (3.8). In turn, by writing the second equation in (5.9) in terms of the Eulerian parts as

$$
\mathbf{b}_{\tau}-\mu_{0} \mathbf{h}_{\tau}-\frac{\mu_{0}}{\zeta}\left(\mathbf{h}_{\tau}-\mathbf{h}_{\tau}^{\text {app }}\right)=0
$$

we simply obtain the constitutive relation $(2.3)$, with

$$
\frac{1}{\zeta}\left(\mathbf{h}_{\tau}-\mathbf{h}_{\tau}^{\mathrm{app}}\right)=\mathbf{m}
$$

This is achieved since $\mathbf{h}_{\tau} \rightarrow \mathbf{h}_{\tau}^{\text {app }}$ as $\zeta \rightarrow 0$ making the first term finite and equal to $\mathbf{m}$. Again, the reader is referred to Danas (2017) for more details on this part.

\subsection{Numerical implementation aspects}

The augmented variational problem (5.8) is solved numerically by use of an implicit, nonlinear finite element (FE) method. The FE computations for the effective RVE response rely on three preliminary steps; (a) RVE generation and conformal meshing, (b) local constitutive modeling along with the local evolution of $\check{\mathcal{H}}^{r}$ and (c) the application of the periodic boundary conditions (5.4). Below we comment on these three points as well as on the actual FE scheme.

$R V E$ generation. This work considers three-dimensional, isotropic RVEs with monodisperse and polydisperse spherical inclusions in a cube having sides of reference length $L_{0}^{\#}\left(\operatorname{such}\right.$ that $\left.\mathcal{V}_{0}^{\#}=\left(L_{0}^{\#}\right)^{3}\right)$. We employ a distance minimizationbased random sequential adsorption (RSA) algorithm that efficiently generates randomly distributed polydisperse spherical inclusions, whose volumes sum up to a prescribed particle volume fraction (Segurado and Llorca, 2002; Anoukou et al., 2018; Zerhouni et al., 2019; Tarantino et al., 2019). For the monodisperse microstructures, we simply prescribe the total number of inclusions in the RVE, which in the present case are equal to $N_{\text {tot }}=60$. Instead for the polydisperse RVE, we consider three families of spherical inclusions of different sizes each with size ratios equal to $1: 7 / 9: 4 / 9$ (from the largest to the smallest). Each family contributes to the total volume fraction in proportions of $0.6 c, 0.3 c$ and $0.1 c$ (from the largest to the smallest). By choosing the largest particle radius to be $R_{\max } / L_{0}^{\#}=0.106$ the remaining two particle families have a radius of $R / L_{0}^{\#}=0.093,0.074$. Those values lead to a total number of particles in the RVE equal to $N_{\text {tot }} \approx 130,280,300$ for $c=0.1,0.2$ and 0.3 , respectively. It is mentioned here that the use of such a high number of particles leads to very large systems with about $10^{6}$ degrees of freedom. This makes the simulation time prohibitively long even when parallelized given the finite strains and large cyclic magnetic loads. For this reason, whenever that is possible from the point of view of convergence in terms of RVE size, we use the monodisperse microstructures. In this regard, a convergence analysis with respect to the particle number and size ratios has been carried out and the above values were found to be acceptable for maintaining a balance between RVE convergence and simulation time. This point is further discussed in the following section. 
Meshing. Conformal meshes of ten-node, isoparametric, quadratic tetrahedral elements are then generated by employing the open source mesh generation software NETGEN (Schöberl, 1997) that also identifies the opposite boundary nodes, which are then used to apply the periodic boundary conditions across the RVE. The reader is referred to the Appendix B of Danas (2017), which provides a straightforward way to apply the magneto-mechanical periodic boundary conditions across the opposite boundaries of the RVE.

Local evolution of internal variable $\breve{\mathcal{H}}^{r}$. A typical FE numerical routine evaluates the local force (generalized force in case of the MREs having both mechanical and magnetic components) vector and stiffness matrix at the individual integration (Gauss) points inside an element. In addition, the proposed internal variable formulation necessitates the storage and evolution of the internal variable $\breve{\mathcal{H}}^{r}$ at each Gauss point. Thus, given the local primary variables $\breve{\mathbf{F}}_{\tau}$ and $\check{\mathbf{H}}_{\tau}$ the local increment in $\breve{\mathcal{H}}^{r}$ from the previously stored $\breve{\mathcal{H}}_{t}^{r}$ is obtained by solving (4.24), which, in turn, leads to the associated switching rule (4.15). Equation (4.15) along with the Kuhn-Tucker conditions therein are solved via an implicit Newton-Raphson method.

Solution of the global optimization problem with the augmented potential energy. Along with the locally-updated $\breve{\mathcal{H}}^{r}$ the local force and stiffness matrices are then constructed, which are subsequently assembled to construct the corresponding global matrices. Finally, the augmented potentials in (5.8) are taken into account by introducing a dummy element that is associated with the corner nodes of the RVE. The local element matrices are constructed by developing a user-defined element (UEL) which is then coupled with the commercial ABAQUS/Standard solver in order to solve the global problem. The inbuilt fully implicit nonlinear FE solver is employed. Finally, the effective incremental response is computed via (5.3) and the volume average of the local $\breve{\mathbf{S}}_{\tau}$ and $\breve{\mathbf{B}}_{\tau}$ fields. The reader is referred to Chapter 4 of the thesis manuscript of Mukherjee (2020) for a more detailed discussion of these steps.

\section{Results: assessment of the analytical model}

This section discusses the model calibration followed by comparisons with the corresponding numerical RVE results under coupled magneto-mechanical loading conditions. In all subsequent results, we use a standard incompressible Neo-Hookean energy for the polymer matrix phase, i.e.,

$$
\rho_{0} \Psi_{\mathrm{m}}^{\mathrm{mech}}\left(I_{1}\right)=\frac{G_{\mathrm{m}}}{2}\left(I_{1}-3\right) .
$$

This functional form is used in (4.2) to obtain the effective mechanical energy for the analytical model. We recall that in the analytical model the mechanical response of the particles is considered rigid. In turn, the magnetic properties of the particle are reported in Table 2 and correspond to a commercially available $\mathrm{NdFeB}$ material. In particular, these parameters are obtained by fitting the purely magnetic model with the experimentally measured hysteresis loops of magnetically isotropic NdFeB particles reported in Deng et al. (2015). Evidently, the model is general enough to be able to deal with any other type of hard magnetic particles.

Table 2: Magnetic properties of the NdFeB particles

\begin{tabular}{|ccccc|}
\hline$\chi_{\mathrm{p}}^{e}$ & $\chi_{\mathrm{p}}^{r}$ & $\mu_{0} m_{\mathrm{p}}^{s}(\mathrm{~T})$ & $b_{\mathrm{p}}^{c}(\mathrm{~T})$ & $\mu_{0}\left(\mu \mathrm{N} \cdot \mathrm{A}^{2}\right)$ \\
\hline 0.105 & 8.0 & 0.842 & 1.062 & $4 \pi 10^{-1}$ \\
\hline
\end{tabular}

The numerical simulations use the same functions and parameters as the analytical model with only two differences that do not affect, however, the validity of the comparison. The first difference is the use of a quasi-incompressible energy for both the matrix and the particle phase, which is simply obtained by adding compressible terms in (6.1), such that it becomes

$$
\rho_{0} \Psi_{\text {comp }, \mathrm{i}}^{\mathrm{mech}}\left(I_{1}, J\right)=\frac{G_{\mathrm{i}}}{2}\left(I_{1}-3-2 \ln J\right)+\frac{G_{\mathrm{i}}^{\prime}}{2}(J-1)^{2}, \quad \text { i }=\mathrm{m}, \mathrm{p} .
$$

The quasi-incompressible character of the matrix is ensured by setting $G_{\mathrm{m}}^{\prime}=500 G_{\mathrm{m}}$. Use of higher values has shown practically no difference in the simulated effective results. The second difference is the use of finite but large Lamé moduli for the particle, i.e., $G_{\mathrm{p}}=500 G_{\mathrm{m}}$ and $G_{\mathrm{p}}^{\prime}=500 G_{\mathrm{p}}$. The phase contrast ratio of $G_{\mathrm{p}} / G_{\mathrm{m}}=500$ has been shown in several earlier studies (see for instance Lopez-Pamies et al. (2013)) to be sufficiently large and thus render a nearly rigid mechanical response for the particle.

The results in the following sections consider the variation of two parameters, namely, the particle volume fraction $c$ and the matrix shear modulus $G_{\mathrm{m}}$, which are, in fact, the two critical parameters that can be varied during the fabrication of $h$-MREs. Instead, the magnetic properties of the particle phase are kept constant.

\subsection{Symmetric cyclic loading and calibration of the $\beta$ function in the model}

In this section, the goal is to calibrate the coupling parameter $\beta$ introduced in the coupled magneto-mechanical energy (4.8) for the analytical model by use of corresponding RVE simulations. For this purpose, we fix the matrix shear modulus to $G_{\mathrm{m}}=0.5 \mathrm{MPa}$ and vary the particle volume fraction $c=0.1,0.2$ and 0.3 . The proposed shear modulus 
resembles closely that of the moderately-soft, commercially-available Sylgard-184 PDMS elastomer (Park et al., 2018; Wang et al., 2019).

We consider symmetric cyclic magnetic loading in terms of $\mathbf{h}^{\text {app }}=h_{1}^{\text {app }} \mathbf{e}_{1}$ with a maximum amplitude $h_{1}^{\text {app }}=3 m_{\mathrm{p}}^{s}$. Note that the loading rate does not play any role here, since both the macro and microscopic $h$-MRE models are rate-independent. As mechanical boundary conditions we employ

$$
S_{11}^{\text {mech }}=S_{22}^{\text {mech }}=S_{33}^{\text {mech }}=0, \quad F_{i j}=0, \quad \forall i \neq j .
$$

Similar to the numerical RVE results of non-hysteretic $s$-MREs (Mukherjee et al., 2020), in $h$-MREs too, the effective magnetostriction response exhibits a certain variance with respect to the RVE realizations, even for sufficiently large number of polydisperse spherical inclusions. For the effective RVE half-cycle responses shown in Fig 4, we employ five different RVE realizations per particle volume fraction $c=0.1,0.2$ and 0.3 . The corresponding average magnetization, $m_{1} / m_{\mathrm{p}}^{s}$ and the parallel, $\lambda_{1}-1$ and transverse, $\lambda_{2,3}-1$, magnetostrictions are shown in Fig. 4 . The light-colored patches around the respective averages indicate the scatter resulting from the considered realizations.
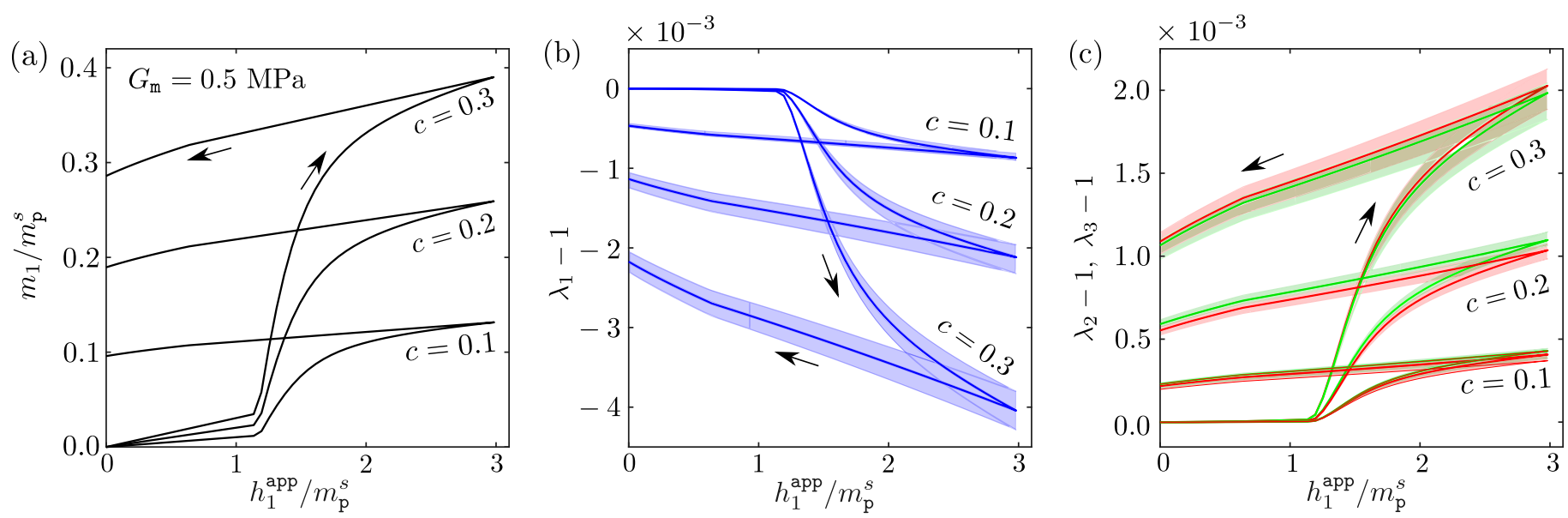

Figure 4: Numerically computed effective (a) magnetization, (b) parallel and (c) transverse magnetostrictions the $h$-MRE RVEs, subjected to uniaxial Eulerian $\mathbf{h}^{\text {app }}=h_{1}^{\text {app }} \mathbf{e}_{1}$ loading/unloading. The average effective responses (solid lines) along with the range of their fluctuations (light patches) for different realizations of the respective RVEs are indicated. The RVEs of different volume fractions are comprised of random polydisperse spherical hard-magnetic inclusions, coming from three distinct families.

Specifically, in Fig. 4a, the scatter of the magnetization response is found to be vanishingly small, whereas, those of the parallel ( $\lambda_{1}$ in Fig. 4b) and transverse magnetostrictions ( $\lambda_{2}$ and $\lambda_{3}$ in Fig. 4c) are gradually increasing with the magnetic load. Notice from Fig. 4 that neither the effective magnetization, nor the magnetostriction saturates at higher $h$-fields. Rather, they maintain a slope with the applied $h_{1}^{\text {app }}$. Such response can be attributed to the inherent non-saturating magnetization response of the $\mathrm{NdFeB}$ particles, as observed in Fig. $2 \mathrm{~b}$ for $\chi_{\mathrm{p}}^{e}>0$. Moreover, we observe that the overall amplitude of the magnetostriction is rather small $\left(\sim 10^{-3}\right)$ indicating that a matrix with shear modulus $G_{\mathrm{m}}=0.5 \mathrm{MPa}$ is rather stiff in relation to the magnetic particle-to-particle forces. Even so, a permanent deformation is obtained upon complete removal of the applied magnetic field. This is obviously a direct consequence of the permanent magnetization of the particles and of their mutual interaction once magnetized permanently.

In Fig. 5a-c, we show the contours of the microscopic $\breve{b}_{1} / \mu_{0} m_{\mathrm{p}}^{s}$ fields after the end of the initial half-cycle (i.e., final state shown in Fig. 4) in the deformed RVEs for the three particle volume fractions under consideration. Figure 5a-c also shows that the magnetic self-fields under no applied $\mathbf{h}^{\text {app }}$ become considerably stronger with increasing volume fraction. Furthermore, the contours of microscopic $\check{m}_{1} / m_{\mathrm{p}}^{s}$ are shown in Fig. 5d-f, where we observe $\check{m}_{1} \approx m_{\mathrm{p}}^{s}$ in the particles, while $\breve{m}_{1}=0$ in the non-magnetic matrix phase. In accord with the computed effective magnetostrictions in Fig. $4 \mathrm{~b}$ and c, we observe very small overall deformation of the RVEs, although the local (microscopic) strain fields may be much higher (twice as large) and varying extensively in the matrix phase. For instance, the contours of the local nominal strain $\breve{\lambda}_{1}-1$ fields are shown in Fig. 5g-i.

The magnetization and magnetostriction response under a fully reversed proportional loading is then computed for $c=0.1,0.2$ and 0.3 by considering a single RVE of each volume fraction. These RVEs are selected to be those, whose effective response is the closest to the corresponding average shown by the firm lines in Fig. $4 \mathrm{~b}$ and c. The resulting magnetization and magnetostriction hysteresis loops along with the corresponding model estimates are shown in Fig. 6. Specifically, one of the magnetization RVE curves $(c=0.3)$ is used to calibrate the two exponents $q_{\text {sat }}$ and $q_{\text {coer }}$ introduced in relations (4.6) and (4.12), respectively ${ }^{9}$. In turn, the RVE magnetostriction curves in Fig. $4 \mathrm{~b}$ are used to calibrate the $\beta$ function introduced in (4.8), which becomes

$$
\beta(c):=19.0 c^{2}-10.4 c+1.71
$$

\footnotetext{
${ }^{9}$ We note that these exponents serve to fine-tune the calibration of the magnetization response allowing for an excellent agreement. Instead, setting them equal to unity changes only weakly the resulting magnetization response.
} 
(a)

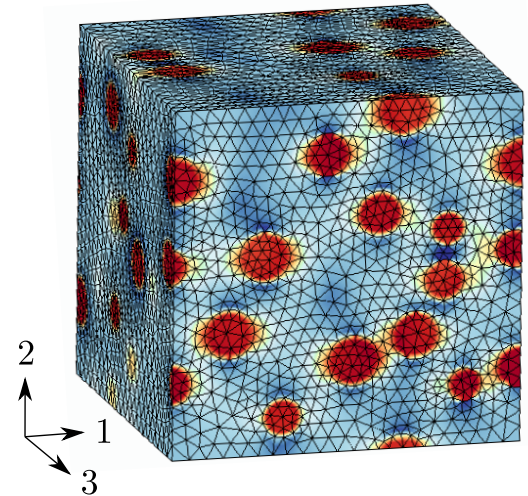

(d)

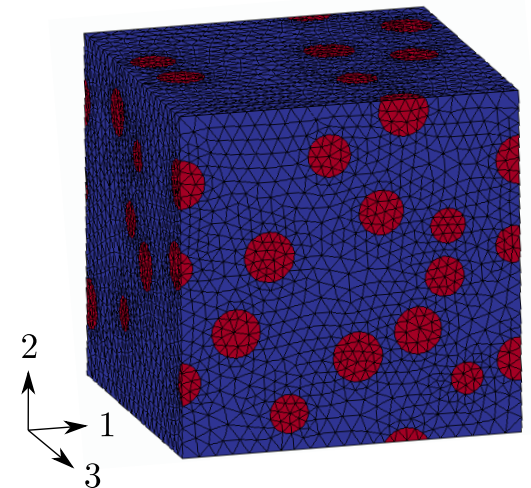

(g)

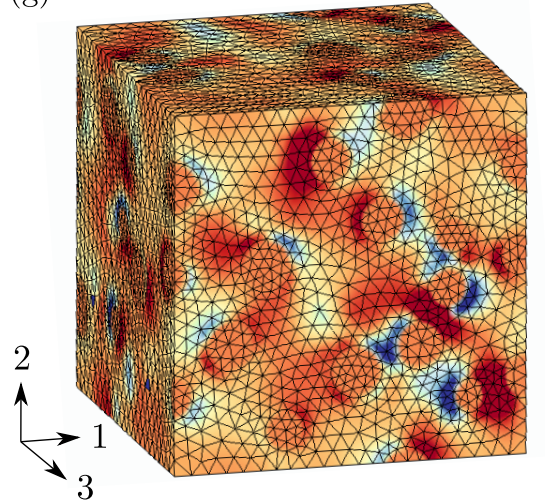

(b)

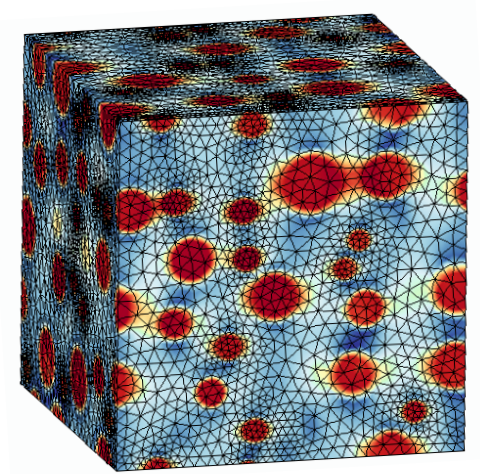

(e)

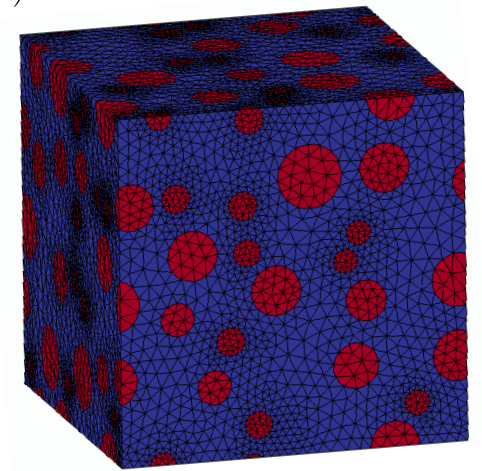

(h)

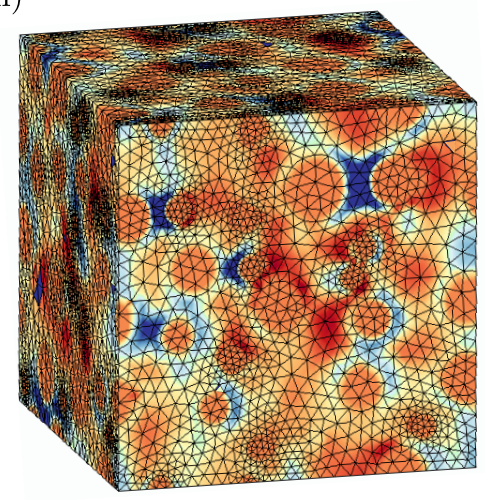

(c) $c=0.3$

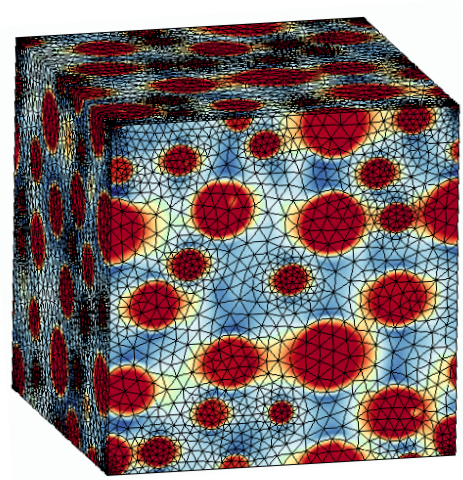

$\check{b}_{1} / \mu_{0} m_{\mathrm{p}}^{s}$

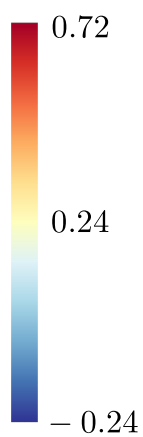

(f)

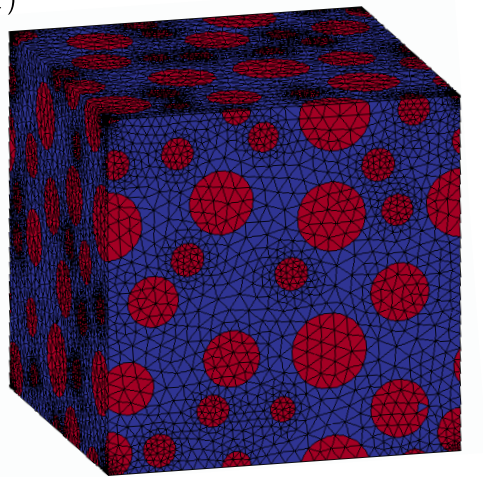

$\check{m}_{1} / m_{\mathrm{p}}^{s}$

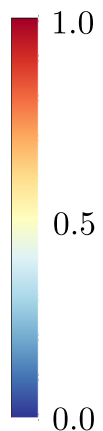

(i)

$\check{\lambda}_{1}-1$

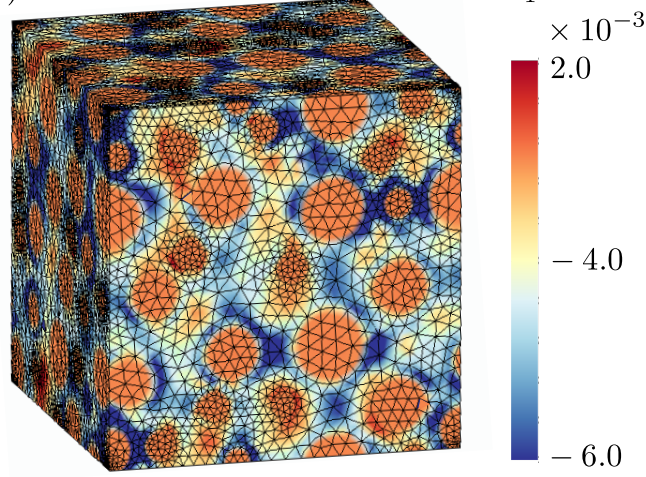

Figure 5: Contours of the numerically computed (a-c) normalized microscopic $\breve{b}_{1}$, (d-f) normalized local $\check{m}_{1}$ and (g-i) nominal mechanical strain $\check{\lambda}_{1}-1$ in the RVEs after the first half cycle of loading along $\mathbf{e}_{1}$, under which the effective responses are shown in Fig. 4 . Three different, RVEs having $(\mathrm{a}, \mathrm{d}, \mathrm{g}) c=0.1,(\mathrm{~b}, \mathrm{e}, \mathrm{h}) 0.2$ and $(\mathrm{c}, \mathrm{f}, \mathrm{i}) 0.3$ are shown.

The range of validity of this function pertains to its calibration range, i.e., $c \leq 0.3$. In addition, we note that $\beta$ is a constant for a given volume fraction and thus only affects the amplitude of the magnetostriction response of the model but not its form, which is of a butterfly type and results directly from the corresponding magnetic hysteresis response.

In this regard, we find that the model is capable of reproducing extremely well the effective magnetic response of the $h$-MRE for several volume fractions. As a result of this excellent agreement, the effective magnetostriction is also well reproduced by only a single calibration constant since $\beta$ is a constant for a given volume fraction $c$.

Finally, we note that as the volume fraction of the particles decreases, the magnetization tends to saturate faster. By contrast, the switching point controlled by the magnetic coercivity $b^{c}$ of the composite seems to be almost insensitive to the particle volume fraction, which justifies the proposition (4.12). We further note that the calibrated $\beta$ parameter in (6.4) is also found to predict the effective magnetostriction responses considerably well for all $G_{\mathrm{m}} \geq 0.2 \mathrm{MPa}$. Some representative computations to probe this predicting capability of the model have been carried out. These results are not shown here for brevity. Instead, we probe the predictive capability of the model in the following subsection by comparison with pre-magnetized $h$-MRE RVEs subjected to combined magneto-mechanical loadings.

\subsection{Mechanical and magnetic loading of a pre-magnetized RVE}

Several experiments and applications use pre-magnetized h-MRE samples (Kim et al., 2018; Zhao et al., 2019; Ren et al., 2019). The pre-magnetization of the samples is achieved by exposing them once to large magnetic fields near 

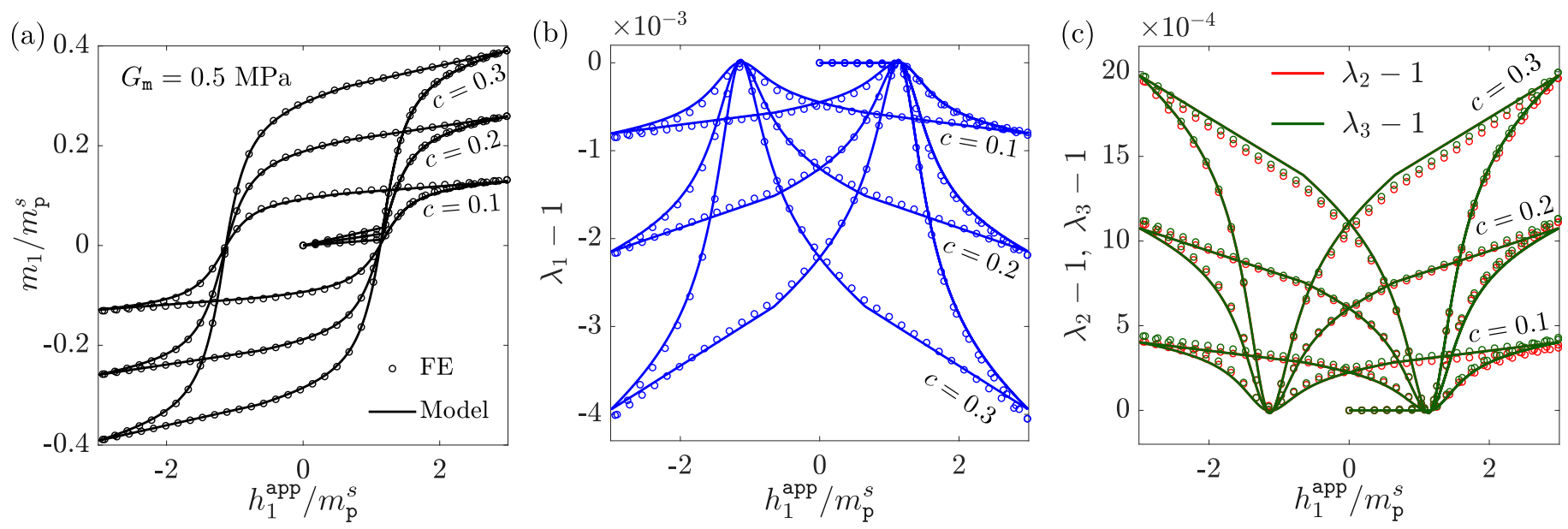

Figure 6: Computed macroscopic hysteresis loops of effective (a) magnetization, (b) parallel and (c) transverse magnetostrictions of $h$-MRE RVEs having the matrix shear modulus $G_{\mathrm{m}}=0.5 \mathrm{MPa}$ and particle volume fractions $c=0.1,0.2$ and 0.3 . The RVEs are subjected to a fully reversed, proportional cyclic loading having amplitude of $3 m_{\mathrm{p}}^{s}$. The average of the effective responses computed from five realizations of a RVE are shown without the fluctuation patches for the magnetostrictions.

magnetic saturation. As already shown in the context of Figs. 4 and 5, this process leads to particles being permanently magnetized.

In this section, we probe the performance of the proposed model in cases of pre-magnetized $h$-MREs. The results are obtained in two steps. In the first step, we pre-magnetize the RVEs starting from the initial state of zero magnetization by applying a magnetic field $h_{1}^{\text {app }} \mathbf{e}_{1}$ up to a value $h_{1}^{\text {app }} / m_{\mathrm{p}}^{s}=3$ and subsequently decreasing it back to zero. This loading procedure is identical to the magnetic half-cycles in Section 6.1. The second step consists in investigating the effects of (i) uniaxial mechanical tension, (ii) simple shear and (iii) cyclic magnetic loading perpendicular to the pre-magnetization direction and assess to model under these loading conditions.

We focus our analysis on pre-magnetized $h$-MREs with particle volume fraction $c=0.2$ and three distinct matrix shear moduli, namely $G_{\mathrm{m}}=0.3,0.5$ and $1 \mathrm{MPa}$. The magnetic material parameters related to the matrix and particle phases remain identical to those in Table 2, whereas the coupling coefficient $\beta$ is considered to be given by (6.4). We also remark that the results shown in the following subsections have been obtained for monodisperse particle distributions in order to make the computations feasible time-wise. Such a simplification is perfectly acceptable for the present loading conditions, where the scatter of the RVE response due to different realization was found to be negligible for monodisperse RVEs with sixty particles.

\subsubsection{Uniaxial tension perpendicular to pre-magnetization}

In this section, we apply a purely mechanical uniaxial tension loading along the $\mathbf{e}_{2}$ direction, which is perpendicular to the direction $\mathbf{e}_{1}$ of the pre-magnetization of the $h$-MRE, while the applied magnetic field is kept identically zero, i.e.,

$$
S_{22}^{\text {mech }} \neq 0, \quad S_{11}^{\text {mech }}=S_{33}^{\text {mech }}=0, \quad F_{i j}=0, \quad \forall i \neq j, \quad \mathbf{h}^{\text {app }}=\mathbf{0} .
$$

As shown in the inset of Fig. 7a, we consider half a cycle, described by a linear increase of $S_{22}^{\text {mech }}$ from 0 to $G_{\mathrm{m}}$ and subsequent decrease to 0 (note that the rate of loading is inconsequential since the models under study are rate-independent). Moreover, we show results for three shear moduli, $G_{\mathrm{m}}=0.3,0.5,1.0$.

Fig. 7a, b and c show the mechanical stretch $\lambda_{2}$ and the magnetizations along the $\mathbf{e}_{1}$ and $\mathbf{e}_{2}$ directions for the numerical RVE and the analytical model. The corresponding deformed RVEs are depicted in Fig. 7d-f. It is noted that the numerically computed effective stretch $\lambda_{2}-\lambda_{2}^{0}$ (with $\lambda_{2}^{0}$ denoting the initial remanent stretch due to the premagnetization) does fluctuate with the different RVE realizations. Nevertheless, as shown in Fig. 4, the magnitude of such realization-dependent scatter in the strain remain less than $5 \times 10^{-4}$, which is considerably smaller than the magnitude of the stretch ( 0.25$)$, shown in Fig. 7a. Thus, the numerical computations with the monodisperse RVEs lead to excellent estimates under purely mechanical loading conditions, while at the same time, they keep the computational expense considerably low. This observation has already been done in the context of $s$-MREs by Danas (2017), where the mechanical and magnetic response was found to converge for RVEs of considerably lower number of particles, whereas the pure magnetostriction (i.e. for zero overall applied mechanical load) required substantially larger RVE sizes with more particles.

In particular, we observe excellent agreement between the numerical homogenization results and the model estimates in all cases shown in Fig. 7a-c, namely, the principle stretch $\lambda_{2}$ and the effective magnetization responses along $\mathbf{e}_{1}$ and $\mathbf{e}_{2}$. The model predictions for the transverse stretches $\lambda_{2}$ and $\lambda_{3}$ also match perfectly the numerically computed responses (not shown explicitly here). It is noted further that all results shown here are independent of the matrix shear modulus upon the normalization $S_{22}^{\text {mech }} / G_{\mathrm{m}}$. This is a particular feature of the Neo-Hookean model used for the mechanical 

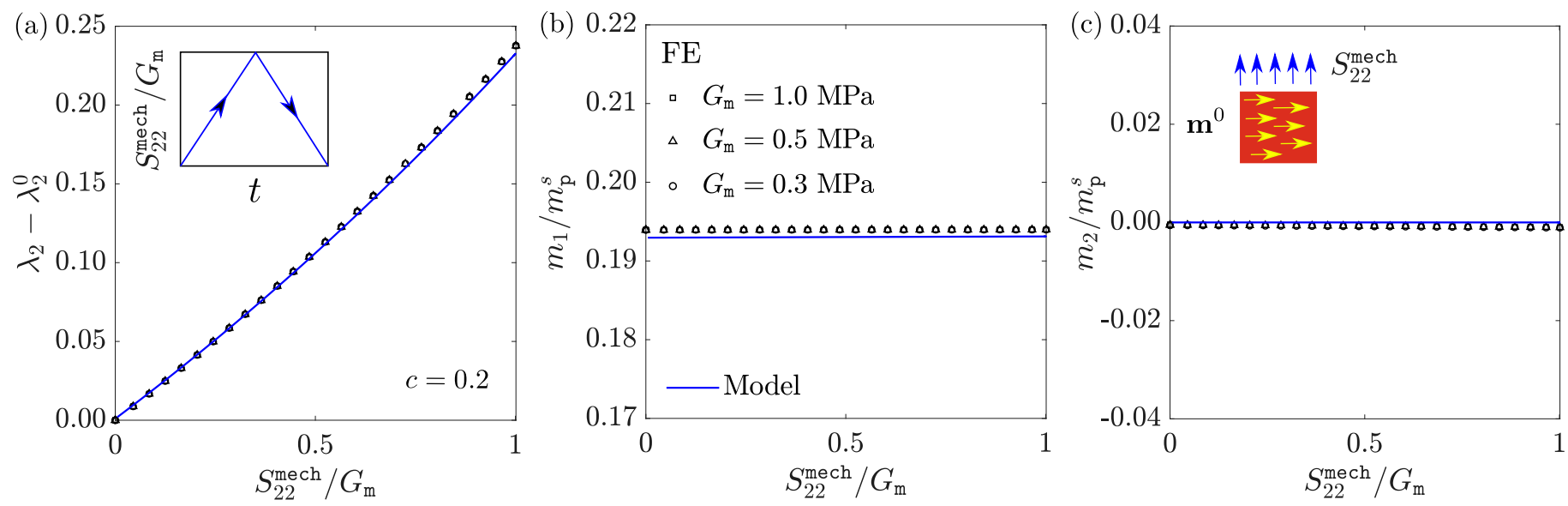

(d) $G_{\mathrm{m}}=1.0 \mathrm{MPa}$

(e)

$$
G_{\mathrm{m}}=0.5 \mathrm{MPa}
$$

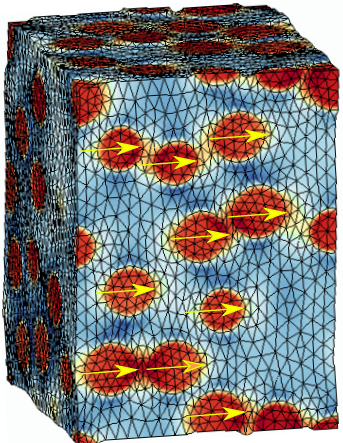

(f)

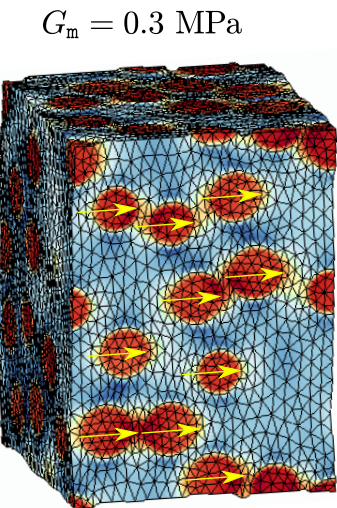

$\check{b}_{1} / \mu_{0} m_{\mathrm{p}}^{s}$

0.92

0.30

$-0.32$

Average direction of $\check{\mathbf{m}}$ in the particles

Figure 7: Evolutions of (a) mechanical stretch $\lambda_{2}-\lambda_{2}^{0}$ (with $\lambda_{2}^{0}$ denoting the initial remanent stretch due to the pre-magnetization) and magnetizations along (b) $\mathbf{e}_{1}$ and (c) $\mathbf{e}_{2}$ under applied uniaxial tensile stress $S_{22}^{\text {mech }}$, whose temporal evolution is shown in the inset of (a). The inset of (c) shows a schematic of the $h$-MRE with the direction of pre-magnetization $\mathbf{m}^{0}$ and the applied uniaxial tension. Contours of the

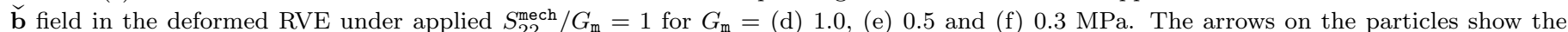
average direction of $\widetilde{\mathbf{m}}$ in them.

description of the matrix phase and simply implies that the overall response of the $h$-MRE is also of a Neo-Hookean type (see relevant discussion in Lopez-Pamies et al. (2013)).

Remark 4. Finally, we close the discussion of Fig. 7 with an important observation, that of the stretch-independence of the current effective remanent magnetization $\mathbf{m}^{0}$, observed in Fig. 7b,c, as predicted by the model and confirmed by the RVE simulations. In simple words, we find that the current remanent magnetization remains unaffected by the stressing (or stretching) of the solid. As a result, the cyclic loading of a pre-magnetized $h$-MRE does not lead to dissipation. This does not mean that the local magnetization does not change via corresponding particle rearrangement. On the contrary, particles rearrange due to the finite straining. Nonetheless, this does not affect the average current magnetization of the RVE, which is an important feature that needs to be reproduced both by phenomenological top-down as well as homogenization bottom-up models. The ability of the present model to recover this feature is linked to the definition of the internal variable $\mathcal{H}^{r}$ in the intermediate stretch-free configuration as discussed in Section 3.1. In turn, this feature has also been observed in the context of $s$-MREs experimentally (Danas et al., 2012), numerically (Mukherjee et al., 2020) and theoretically (Lefèvre et al., 2017) via an independence of the current magnetization response to pre-stressing. This feature is linked also to the underlying (quasi-)incompressibility of the materials under study and should be taken into account in the modeling of MREs in general.

\subsubsection{Simple shear parallel to pre-magnetization}

We apply a purely mechanical simple shear stress $S_{12}^{\text {mech }}$ loading. The corresponding traction vector is parallel to the pre-magnetization direction $\mathbf{e}_{1}$ of the $h$-MRE, while the applied magnetic field is kept identically zero during this step, i.e.,

$$
S_{12}^{\text {mech }} \neq 0, \quad S_{11}^{\text {mech }}=S_{22}^{\text {mech }}=S_{33}^{\text {mech }}=0, \quad F_{21}=F_{13}=F_{31}=F_{23}=F_{32}=0, \quad \mathbf{h}^{\text {app }}=\mathbf{0} .
$$

Furthermore, the evolution for the applied $S_{12}^{\text {mech }}$ is shown in the inset of Fig. 8a.

In Fig. 8a-c, we observe an excellent agreement between the model predictions and the numerical homogenization results for the effective shear strain $F_{12}=\gamma_{12}$ as well as for the effective magnetizations along $\mathbf{e}_{1}$ and $\mathbf{e}_{2}$, respectively. All results, shown in the context of this figures, are independent of the matrix shear modulus upon the normalization $S_{12}^{\mathrm{mech}} / G_{\mathrm{m}}$. 

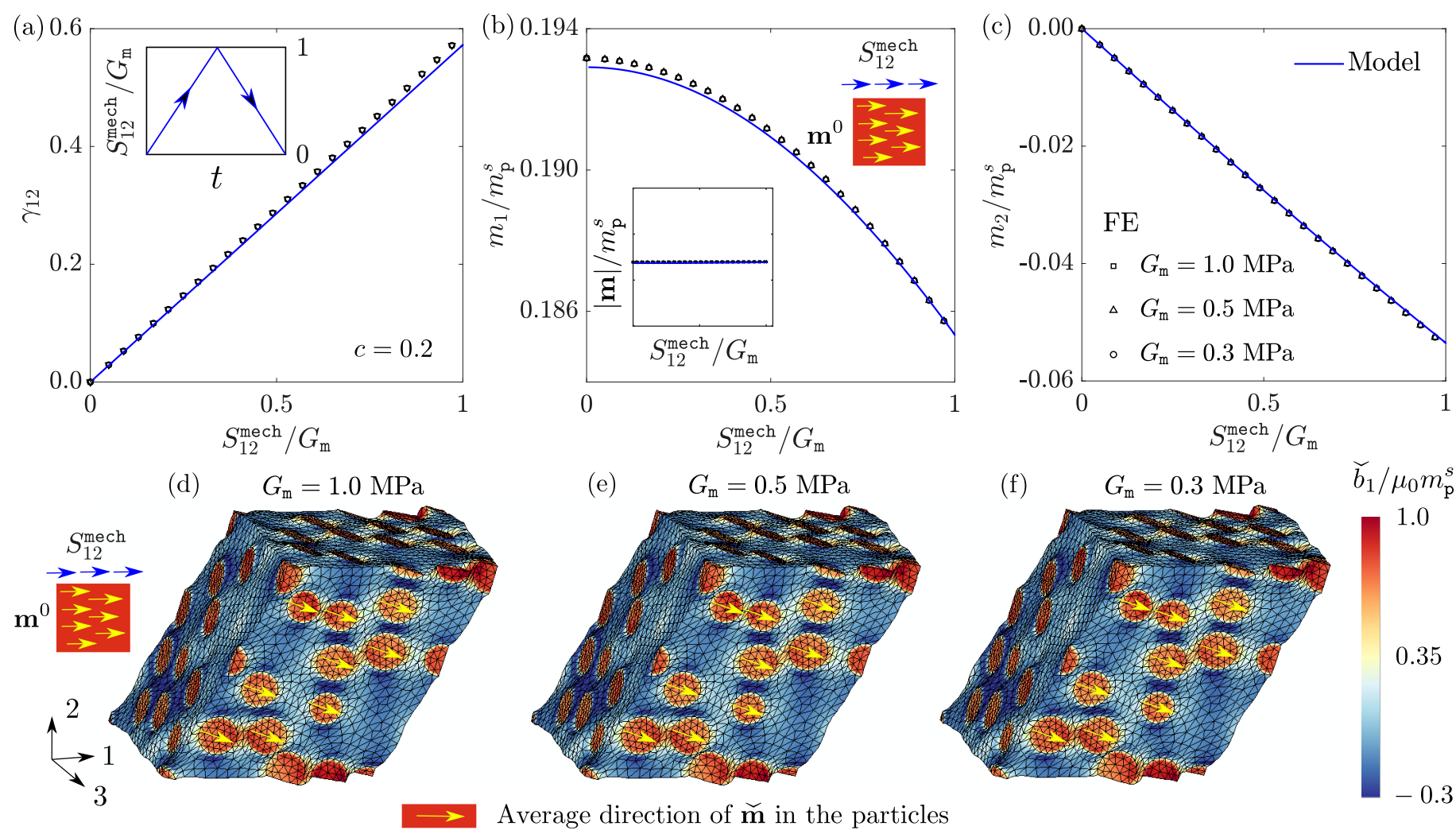

Figure 8: Evolutions of (a) shear strain $\gamma_{12}$ and magnetizations along (b) $\mathbf{e}_{1}$ and (c) $\mathbf{e}_{2}$ under applied simple shear stress $S_{12}^{\text {mech }}$, whose loading path is shown in the inset of (a). A schematic of the $h$-MRE with the direction of pre-magnetization $\mathbf{m}^{0}$ and the applied shear stress is shown in the inset of (b). Contours of the $\breve{\mathbf{b}}$ field in the deformed RVE under applied $S_{12}^{\text {mech }} / G_{\mathrm{m}}=1$ for $G_{\mathrm{m}}=$ (d) 1.0 , (e) 0.5 and (f) $0.3 \mathrm{MPa}$. The arrows on the particles show the average direction of $\widetilde{\mathbf{m}}$ in them.

Again, we observe that despite the significant shearing strains and particle rearrangements, the amplitude of the current effective magnetization $\mathbf{m}$ remains unaffected (see inset of Fig. 8b). Instead, the orientation of the magnetization vector significantly changes with the applied shearing, as revealed by the change of the individual components $m_{1} / m_{\mathrm{p}}^{s}$ and $m_{2} / m_{\mathrm{p}}^{s}$ in Fig. $8 \mathrm{~b}$ and c, respectively. Interestingly, this rotation remains (almost) identical to the macroscopic (average) rotation of the RVE induced by the shearing. Therefore, the affine rotation-based model presented in this work predicts the evolution of $\mathbf{m}$ in this case accurately.

Figures 8d-f show three deformed RVEs at $S_{12}^{\text {mech }} / G_{\mathrm{m}}=1$ for the three $G_{\mathrm{m}}$ under consideration. It is noted that the RVE deformations and the local $\breve{b}_{1}$ fields remain identical for all three $G_{\mathrm{m}}$ under consideration. The only key feature to note here is the uniform distribution of the particle rotations, which, in turn, rotates the local (microscopic) and therefore the global (effective) magnetization vectors.

\subsubsection{Cyclic magnetic loading perpendicular to pre-magnetization}

In this section, we investigate the effect of cyclic magnetic loading applied along $\mathbf{h}^{\text {app }}=h_{2}^{\text {app }} \mathbf{e}_{2}$, which is perpendicular to the direction of the pre-magnetization $\mathbf{e}_{1}$. As shown in the inset of Fig. 9a, the loading/unloading path is a simple ramp-type linear increase/decrease from a zero applied field to $h_{2}^{\text {app }}=3 m_{\mathrm{p}}^{s}$ and then decrease to zero. The mechanical boundary conditions for this loading case are given by

$$
S_{11}^{\text {mech }}=S_{22}^{\text {mech }}=S_{33}^{\text {mech }}=S_{12}^{\text {mech }}=S_{32}^{\text {mech }}=0, \quad F_{21}=F_{13}=F_{31}=F_{23}=0 .
$$

Since all the shear degrees of freedom on the surface with reference unit normal along $\mathbf{e}_{2}$ are left free, the applied magnetic loading induces an average shear strain along direction 1, namely $\gamma_{12}$, in the RVE due to the collective rotation of the microscopic hard magnetic particles. The evolution of the magnetization components along $\mathbf{e}_{1}$ and $\mathbf{e}_{2}$ along with that of the induced shear under applied $h_{2}^{\text {app }}$ are shown in Fig. 9. Unlike the preceding two results involving mechanical tension and shear of a pre-magnetized $h$-MRE, here we observe a strong dependence of the resulting magneto-mechanical response on $G_{\mathrm{m}}$. First, we notice from Fig. 9c, f and i that the induced shear strain increases with the decreasing shear modulus of the matrix. Furthermore, the magnitude of the induced shear strains are one to two orders higher than those of the magnetostrictions under a aligned magnetic loading, as observed in Fig. $4 \mathrm{~b}$ and c. This last observation suggests that the effect of collective particle rotations due to non-aligned magnetic loading induces a substantially stronger magnetomechanical coupling than inter-particle attraction. This is perhaps the reason that most of the soft robotic applications of $h$-MREs exploit these large deflections under non-aligned magnetic loadings with respect to the pre-magnetization 

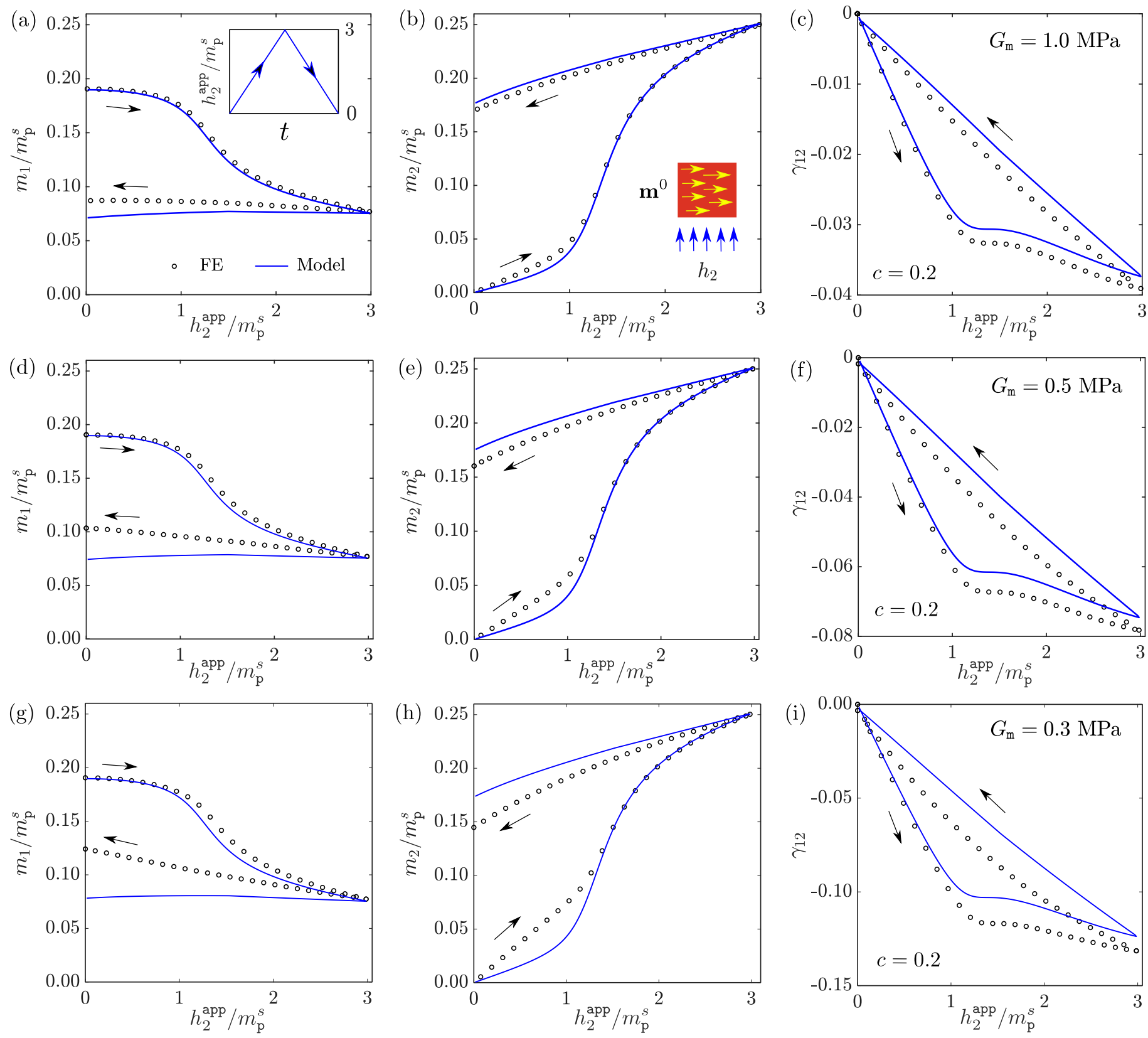

Figure 9: Evolution of the magnetization components along $\mathbf{e}_{1}(\mathrm{a}, \mathrm{d}, \mathrm{g})$ and $\mathbf{e}_{2}(\mathrm{~b}, \mathrm{e}, \mathrm{h})$ and the induced shear strain $\gamma_{12}$ (c,f,i) under applied perpendicular magnetic loading along $\mathbf{e}_{2}$ (inset of a) to a pre-magnetized $h$-MRE along $\mathbf{e}_{1}$ (inset of b). Effect of three distinct $G_{\mathrm{m}}$, namely, $G_{\mathrm{m}}=(\mathrm{a}-\mathrm{c}) 1.0,(\mathrm{~d}-\mathrm{f}) 0.5$ and $(\mathrm{g}-\mathrm{i}) 0.3 \mathrm{MPa}$ on the effective magnetization components and induced shear strain.
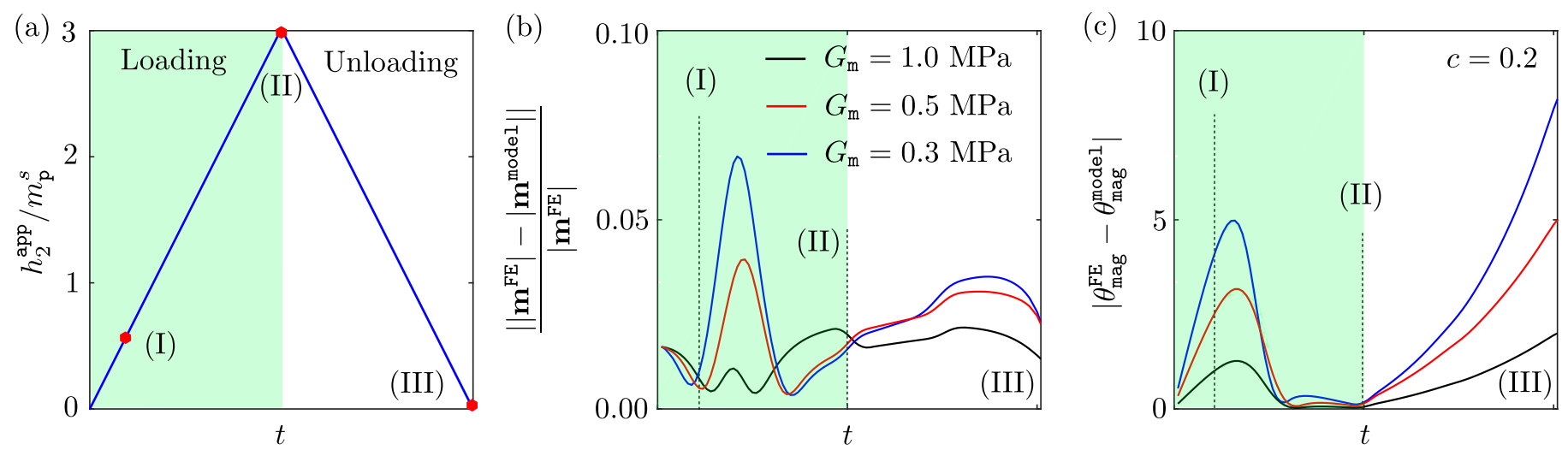

Figure 10: (a) Proportional magnetic loading path along $\mathbf{e}_{2}$, (b) relative difference between the computed and model predicted magnitudes of $\mathbf{m}$ and (c) angle (in an absolute sense) between the computed and the model-predicted effective magnetizations. The points (I), (II) and (III) indicates loading instances at which the deformed RVE along with the $b_{2}$ contours are shown in Fig. 11 
direction (Kim et al., 2018; Zhao et al., 2019). We also note from the numerical homogenization computations that, under this non-aligned loading condition, the scale of variations due to RVE realizations in the magnetostriction stretch components $\lambda_{i}(i=1-3)$ remains less than $2 \times 10^{-3}$ and are significantly smaller than the induced shear strains due to the particle realignment. As a result, such variations do not play a significant role in the macroscopic response of the $h$-MREs, while they allow the use of RVEs with a smaller number of monodisperse particles.

In this highly non-trivial loading scenario, the model predictions remain in acceptable agreement with the computed RVE results, especially for stiffer $h$-MREs having $G_{\mathrm{m}} \geq 0.5 \mathrm{MPa}$. The predicted effective magnetization and shear responses, however, start differing from the numerical ones for the relatively softer $h$-MRE with $G_{\mathrm{m}}=0.3 \mathrm{MPa}$. To further investigate this disparity, we plot the relative error in the magnitude of the predicted magnetization and the predicted direction of the magnetization with respect to the numerical homogenization estimates in Fig. $10 \mathrm{~b}$ and c. In particular, we observe from Fig. 10b that, the relative error in the predicted magnitude of $\mathbf{m}$ remains below $3 \%$ during most of the loading/unloading process. On the other hand, Fig. 10c shows that the absolute difference between $\theta_{\text {mag }}^{\mathrm{FE}}$ and $\theta_{\text {mag }}^{\text {model }}$ becomes significant at the initiation of the non-proportional loading and during the unloading. Here the angles $\theta_{\text {mag }}$ are measured in degrees and with respect to the $X_{1}$ axis and therefore, are computed simply via $\theta_{\operatorname{mag}}=\tan { }^{-1}\left(m_{2} / m_{1}\right)$.
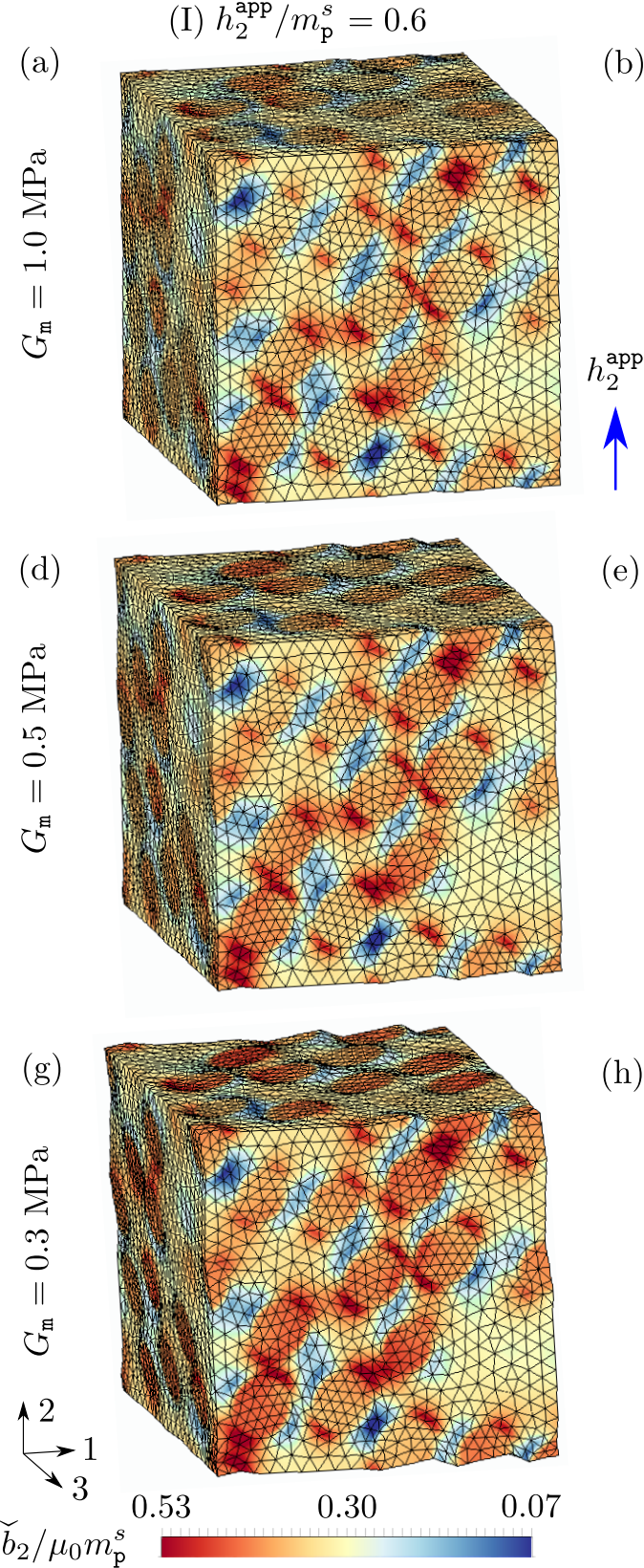

(II) $h_{2}^{\mathrm{app}} / m_{\mathrm{p}}^{s}=3.0$

(b)

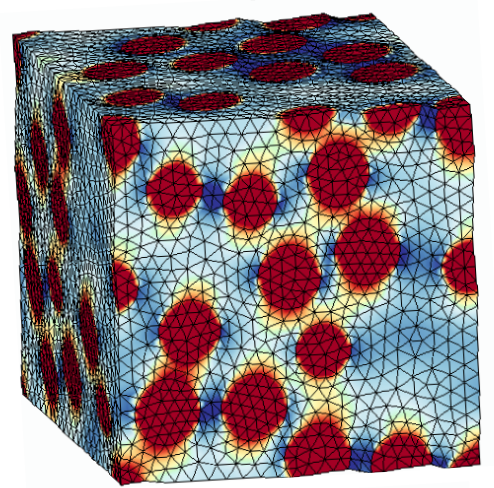

(e)

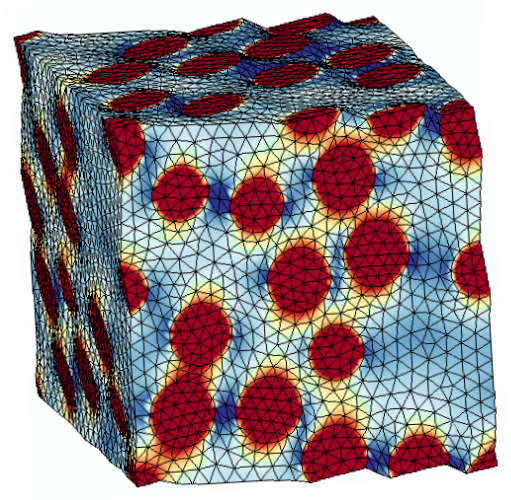

(h)

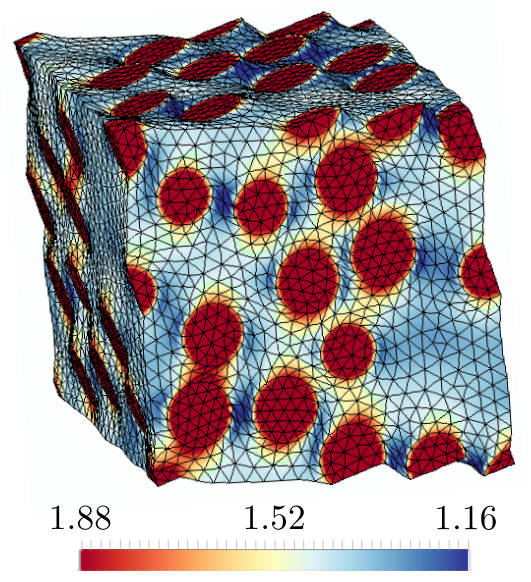

(c)

$$
\text { (III) } h_{2}^{\mathrm{app}} / m_{\mathrm{p}}^{s}=0.0
$$

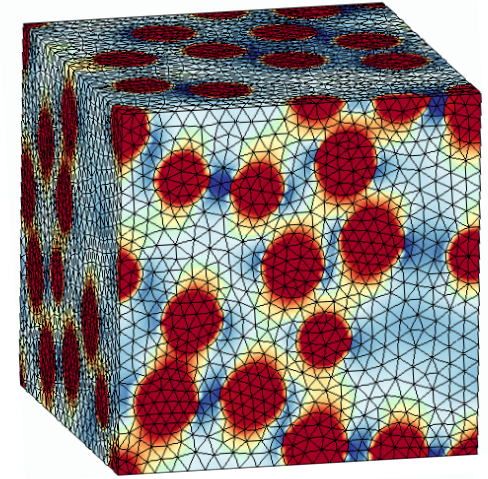

(f)

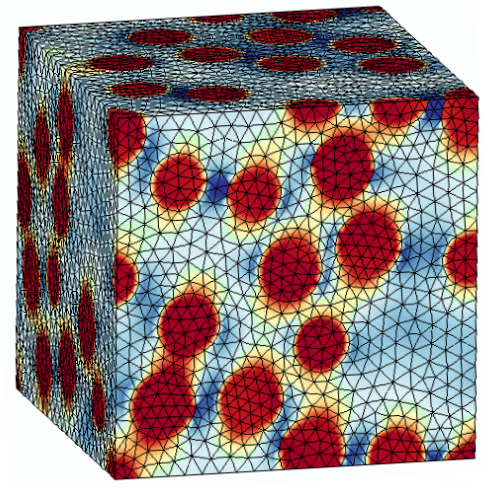

(i)

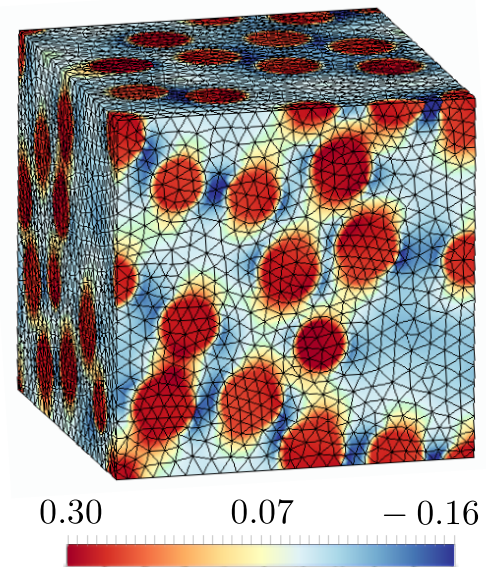

Figure 11: Contours of the numerically computed local $\breve{b}_{2}$ fields in a deformed mesh at the loading/unloading instances (I) (a,d,g), (II) (b,e,h) and (III) (c,f,i) as indicated in Fig. 10a. Contours are shown for three different matrix shear modulus, namely, $G_{\mathrm{m}}=1.0 \mathrm{MPa}$ (a-c), $0.5 \mathrm{MPa}$ (d-f) and $0.3(\mathrm{~g}-\mathrm{i})$.

The increasing differences between the predicted and computed directions of the effective $\mathbf{m}$ for a decreasing matrix shear modulus is attributed to the non-affine particle rotations in the microstructure, i.e., the particles tend to rotate 
differently from the macroscopic rotation of the RVE(Kalina et al., 2017). Instead, the magnetization magnitudes match significantly better. Evidently, the extent of these non-affine rotations increases with decreasing $G_{\mathrm{m}}$, thus, leading to gradually larger differences between the model predictions and the numerical homogenization results. Even so, the predicted induced shear strain responses for $G_{\mathrm{m}}=0.3 \mathrm{MPa}$ in Fig. 9i exhibit an acceptable qualitative and quantitative agreement with the corresponding numerical results, given the strongly non-aligned loading considered here.

In connection with this, Figure 11 shows a number of representative contour plots of the local $\breve{b}_{2}$ fields in the composite at various loading instants, indicated by (I), (II) and (III) on Fig. 10a. Evidently, the deformed configurations in Fig. 11 show a greater extent of induced shear in the RVEs with softer matrix (cf., Fig. 11b and h), which are in accordance with the effective responses in Fig. 9. Furthermore, visual inspections of the individual particles in Fig. 11b, e and h clearly show an increasing tendency of non-proportional particle rotations. Even if the deformed configurations in state (III), i.e., Fig. 11c, f and i look identical, the directions of the local magnetization in the particles differ considerably. This is due to the greater extent of non-affine particle rotations in the softer matrix $\left(G_{\mathrm{m}}=0.3 \mathrm{MPa}\right)$ during the initial non-proportional loading cycle (see initial surface fluctuations in Fig. 10c).

\subsection{Discussion on the non-affine rotation of the particles}

More precisely, the magnetization of $h$-MREs along the direction of non-aligned magnetic loading results from two competing mechanisms: first, from the particle rotations in the soft elastomer matrix and second, from the magnetic switching in the particles themselves. As shown in Fig 10c, during the initial non-aligned loading, the particles are more susceptible to undergo rotations in order to align themselves with the applied $h$-field, thus, inducing an overall shear strain $\gamma_{12}$ in the RVE. Moreover, the tendency of the particles to undergo additional non-affine rotations increases with the decreasing $G_{\mathrm{m}}$. Nevertheless, both affine and non-affine particle rotations are hindered by the elastic restoring energy, exerted by the matrix. This constraint to the (affine and non-affine) particle rotations increases with increasing $G_{\mathrm{m}}$, leading to smaller induced $\gamma_{12}$ and also lesser non-affine rotations (see Fig. 10c). Eventually, the magnetic switching mechanism dominates over the particle rotations at higher applied fields and consequently, the induced shear starts to saturate (see Fig. 9c, f and i). Also, we observe negligibly small non-affine particle rotations in Fig. 10c during the later part of the loading half cycle, when the magnetic switching mechanism dominates in the process of rotating the average m.

During the unloading, on the other hand, no magnetic switching takes place. Nonetheless, because of the elastic restoring energy, the rotated particles start coming back to their initial position as the applied magnetic field $h_{2}$ is gradually removed. Consequently, the induced $\gamma_{12}$ vanishes upon the removal of the applied $h_{2}^{\text {app }}$ (see Fig. 9c, f, i and Fig. 11c, f and i). In addition, the particles also undergo non-affine rotations while returning back to their initial positions, in order to compensate for their non-affine rotations during the initial part of the loading half cycle. Consequently, this leads to the differences between numerical results and the proposed affine rotation-based model predictions, especially at smaller values of the shear modulus of the matrix (Fig. 9g and h).

Even so, the analytical model proposed here is versatile enough to be further calibrated to recover more accurately the response even at smaller values of $G_{\mathrm{m}}$. Such an exercise is not conducted here because that is beyond the scope of the present study. Nevertheless, the model is used in the next section to solve boundary value problems and compare with experiments proposed in the recent literature of $h$-MREs showing its strength by comparison with the extremely time consuming RVE calculations.

\section{Results: solution of macroscopic boundary value problems using the analytical model}

In this section, we employ the analytical model proposed in this study to solve macroscopic boundary value problems (BVPs) with $h$-MREs ${ }^{10}$. Till date, most experimental $h$-MRE studies consider pre-magnetized slender beams under applied non-aligned magnetic fields (Kim et al., 2018; Zhao et al., 2019; Ren et al., 2019). We first solve a macroscopic boundary value problem that models the experiment of Zhao et al. (2019) with a uniformly pre-magnetized cantilever beam. After that, we investigate three cases of non-uniformly magnetized $h$-MRE beams subjected to magnetic loading that is perpendicular to the long axis of the beam. The latter are motivated from the experiments of Ren et al. (2019), although the exact geometric/material parameters are not used here. Both problems are solved in a two-dimensional plane-strain setting for simplicity. The model however is three-dimensional as shown earlier.

\subsection{Deflection of uniformly pre-magnetized cantilever beams}

As pointed out by numerous works with BVPs involving MREs, the magnetic field is applied by fixed magnetic poles, situated far from the MRE sample (Psarra et al., 2017; Bodelot et al., 2017; Zhao et al., 2019). Such condition is adapted in a numerical setting via considering a sufficiently large volume of surrounding air around the $h$-MRE sample (see Fig. 12a). In the framed two-dimensional numerical BVP, the total numerical computation area is considered to be a

\footnotetext{
${ }^{10}$ It is unambiguous here that the present BVPs involve a $h$-MRE sample inside an air domain and the corresponding applied magnetic fields are applied far from the body. Instead, in Section 6, we dealt with RVE simulations and thus infinite domains that involve no air. The applied fields therein may be identified with the average magnetic fields in the RVE.
} 


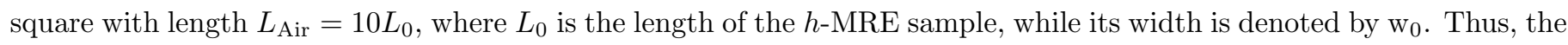
aspect ratio of the rectangular $h$-MREs is defined via $r_{\text {asp }}=L_{0} / \mathrm{w}_{0}$. The total numerical computation area is defined via $\mathcal{V}=\mathcal{V}_{\text {Air }} \cup \mathcal{V}_{\text {MRE. }}$. While the outer boundary of the surrounding air domain is defined via $\partial \mathcal{V}_{\text {Air }}=\partial \mathcal{V}_{\text {Air }}^{\text {Left }} \cup \partial \mathcal{V}_{\text {Air }}^{\text {Top }} \cup \partial \mathcal{V}_{\text {Air }}^{\text {Right }} \cup$ $\partial \mathcal{V}_{\text {Air }}^{\text {Bottom }}$, the interface between the $h$-MRE and the air is defined via $\partial \mathcal{V}_{\text {MRE }}=\partial \mathcal{V}_{\text {MRE }}^{\text {Left }} \cup \partial \mathcal{V}_{\text {MRE }}^{\text {Top }} \cup \partial \mathcal{V}_{\text {MRE }}^{\text {Right }} \cup \partial \mathcal{V}_{\text {MRE }}^{\text {Bottom }}$ (see Fig. 12a and b).

(a)

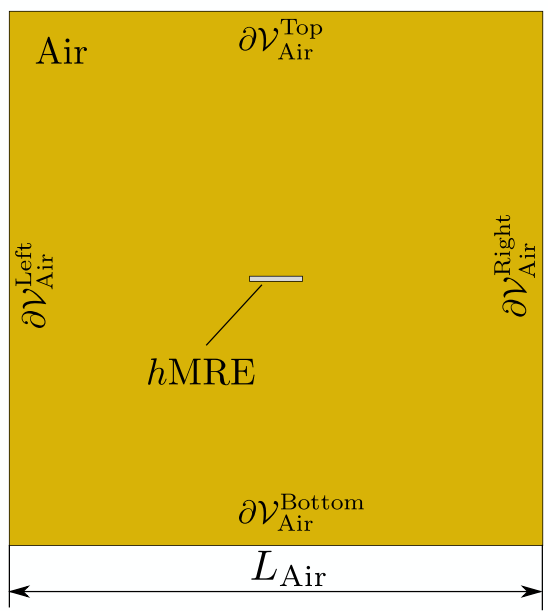

(b) Step 1

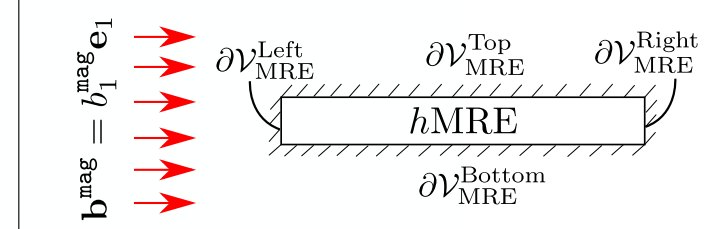

(c) Step 2
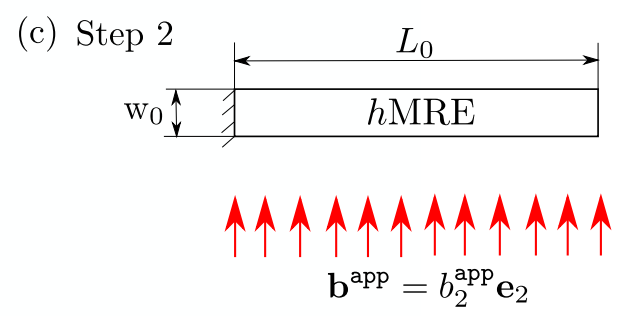

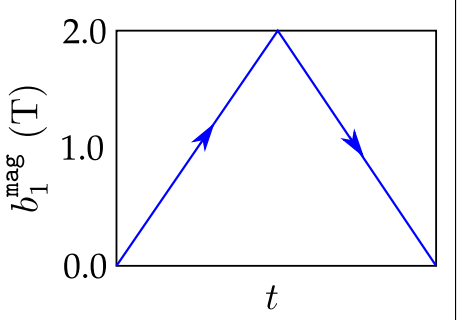

$t$

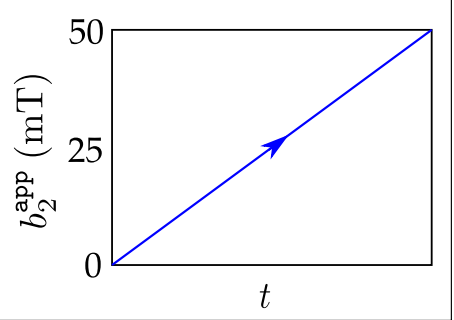

Figure 12: (a) Schematic diagram of the numerical BVP involving the $h$-MRE and surrounding air. The length of the square air domain $L_{\text {Air }}$ is considered to be $L_{\text {Air }}=10 L_{0}$ and four boundaries of this domain are denoted by $\partial \mathcal{V}_{\text {Air }}^{\text {Left }}, \partial \mathcal{V}_{\text {Air }}^{\text {Top }}, \partial \mathcal{V}_{\text {Air }}^{\text {Right }}$ and $\partial \mathcal{V}_{\text {Air }}^{\text {Bottom }}$. (b) Step 1, indicating the applied pre-magnetizing field $\mathbf{b}^{\text {mag }}$ direction (by red arrows) and magnitude (right hand side plot). The four interfaces between air and $h$-MRE are denoted by $\partial \mathcal{V}_{\mathrm{MRE}}^{\mathrm{Left}}, \partial \mathcal{V}_{\mathrm{MRE}}^{\text {Top }}, \partial \mathcal{V}_{\mathrm{MRE}}^{\text {Right }}$ and $\partial \mathcal{V}_{\mathrm{MRE}}^{\text {Bottom }}$. The hatched interfaces represent fixed displacement degrees-of-freedom, i.e. $\mathbf{u}=\mathbf{0}$. (c) Two subsequent magnetic loading paths for (c) Step 2, indicating the actuation field $\mathbf{b}^{\text {app }}$ applied along $\mathbf{e}_{2}$, while its magnitude is shown in the adjacent plot. The length and width of the $h$-MRE are indicated by $L_{0}$ and $\mathrm{w}_{0}$, respectively, while the fixed $h$-MRE-air interface in this step is indicated by a hatched line.

Similar to the microscopic problem, we employ the incremental potential (4.24) in order to numerically obtain the local magnetic and mechanical fields for the $h$-MRE, we employ the quasi-incompressible mechanical model in (4.9) together with the switching surface (4.14) and the associated switching rule (4.15).

The modeling of air space surrounding the $h$-MRE needs special consideration (Keip and Rambausek, 2016; Pelteret et al., 2016; Psarra et al., 2017, 2019). In this work we follow the approach of Psarra et al. (2017, 2019) which is summarized in the following. In order to obtain a numerically feasible "deformation" in the free space, we apply a linear constraint on each node in the air domain $\left(\mathcal{V}_{\text {Air }}\right)$. For this purpose, we first define a distance coefficient for each node in $\mathcal{V}_{\text {Air. }}$. This requires to find the closest node on the interface $\partial \mathcal{V}_{\text {MRE }}$ to the node $n$ in $\mathcal{V}_{\text {Air }}$, which is achieved by a simple nearest neighbor search algorithm. The distance coefficient is subsequently defined to be

$$
d_{i}^{(n)}=1-\frac{\left|X_{i}^{(n)}\right|_{\partial \mathcal{V}_{\mathrm{MRE}}}-\left.X_{i}^{(n)}\right|_{\mathcal{V}_{\mathrm{Air}}} \mid}{0.5 L_{\mathrm{Air}}} .
$$

Subsequently, we employ a constraint between those two nodes, the one at the boundary of the solid and that in the air, which reads

$$
\mathcal{C}_{i}^{(n)} \equiv \begin{cases}\left.d_{i}^{(n)} u_{i}^{(n)}\right|_{\partial \mathcal{V}_{\mathrm{MRE}}}-\left.u_{i}^{(n)}\right|_{\mathcal{V}_{\mathrm{Air}}}=0, & \text { if } 0<d_{i}^{(n)} \leq 1 \\ \left.u_{i}^{(n)}\right|_{\mathcal{V}_{\mathrm{Air}}=0,}=0 & \text { otherwise }\end{cases}
$$

In order to apply this constraint in the present incremental variational setting, we introduce a penalty potential to be applied in the air domain, such that (Psarra et al., 2019)

$$
\rho_{0} \Psi_{\text {penalty }}(\mathbf{u})=\sum_{n=1}^{N_{\text {Air }}} \sum_{i=1}^{2} \frac{G_{c}}{2 L_{c} \zeta}\left(\mathcal{C}_{i}^{(n)}\right)^{2},
$$

where $N_{\text {Air }}$ is the number of air nodes, $L_{c}$ is a reference length parameter set to be equal to $\mathrm{w}_{0}, G_{c}$ is an arbitrary shear modulus-like parameter, which is set to that of the $h$-MRE and $\zeta$ is a penalty parameter, which is set to $10^{-3}$ in order to ensure the proper application of the constraint (7.2).

Finally, the total potential energy associated with the air takes the form

$$
W_{\text {Air }}(\mathbf{u}, \varphi)=\rho_{0} \Psi_{\text {penalty }}(\mathbf{u})-\frac{\mu_{0}}{2} J I_{5}
$$


The second term in this expression is the magnetostatic energy, which must be included in order to assure a finite magnetic $b$-field in the air domain $\mathcal{V}_{\text {Air }}$. No additional mechanical energy is considered for the air. In practice, the penalty potential (7.3) is applied in the finite-element setting by defining a set of $N_{\text {Air }}$ dummy elements having two nodes, namely, the air node and the MRE interface node.

In summary, the incremental variational principle for the BVP becomes in the time step $[t, \tau]$

$$
\mathscr{P}\left(\mathbf{u}_{\tau}, \varphi_{\tau}\right)=\inf _{\mathbf{u}_{\tau} \in \mathcal{K}_{\mathrm{BVP}}} \sup _{\varphi_{\tau} \in \mathcal{G}_{\mathrm{BVP}}}\left[\int_{\mathcal{V}_{\mathrm{MRE}}} \mathcal{W}\left(\mathbf{u}_{\tau}, \varphi_{\tau}\right) \mathrm{d} V+\int_{\mathcal{V}_{\mathrm{Air}}} W_{\mathrm{Air}}\left(\mathbf{u}_{\tau}, \varphi_{\tau}\right) \mathrm{d} V\right] .
$$

Note that mechanical body forces are neglected and no mechanical tractions are applied anywhere in the domain. The incremental potential $\mathcal{W}$ for the $h$-MRE is defined by (4.24). In addition, the constraint sets $\mathcal{K}_{\text {BVP }}$ and $\mathcal{G}_{\text {BVP }}$ are those defined in (5.6) and (5.7), respectively, with the periodic boundary conditions replaced by proper Dirichlet conditions at the $h$-MRE boundary, denoted by $\partial \mathcal{V}_{\mathrm{MRE}} \equiv \partial \mathcal{V}_{\mathrm{MRE}}^{\text {Top }} \cup \partial \mathcal{V}_{\mathrm{MRE}}^{\text {Left }} \cup \partial \mathcal{V}_{\mathrm{MRE}}^{\text {Bottom }} \cup \partial \mathcal{V}_{\mathrm{MRE}}^{\text {Right }}$, and the air boundary, denoted by $\partial \mathcal{V}_{\text {Air }} \equiv \partial \mathcal{V}_{\text {Air }}^{\text {Top }} \cup \partial \mathcal{V}_{\text {Air }}^{\text {Left }} \cup \partial \mathcal{V}_{\text {Air }}^{\text {Bottom }} \cup \partial \mathcal{V}_{\text {Air }}^{\text {Right }}$, as discussed in the following. These Dirichlet boundary conditions in the BVP allow for an initial pre-magnetization along the long axis of the beam and subsequent actuation by applying a magnetic field perpendicular to the pre-magnetization direction. The steps to apply them are:

- Step-1: Pre-magnetization of the beam. As indicated in Fig. 12b (top), the first "pre-magnetization" step is carried out by considering all the $h$-MRE and air boundaries to be fixed, while a magnetic (pre-)loading along the long axis of the beam is applied in terms of the magnetic scalar potential $\varphi$, such that

$$
\begin{aligned}
& \mathbf{u}_{\tau}=\mathbf{0}, \quad \forall \mathbf{X} \in \partial \mathcal{V}_{\mathrm{MRE}} \quad \text { and } \quad \mathbf{u}_{\tau}=\mathbf{0}, \quad \forall \mathbf{X} \in \partial \mathcal{V}_{\text {Air }} \\
& \varphi_{\tau}=0, \quad \forall \mathbf{X} \in \partial \mathcal{V}_{\text {Air }}^{\text {Left }} \quad \text { and } \quad \varphi_{\tau}=-\frac{b_{1, \tau}^{\mathrm{mag}}}{\mu_{0}} L_{\text {Air }} \quad \forall \mathbf{X} \in \partial \mathcal{V}_{\text {Air }}^{\text {Right }} .
\end{aligned}
$$

The temporal evolution of $b_{1}^{\mathrm{mag}}$ is also shown in Fig. $12 \mathrm{~b}$.

- Step-2: Actuation via a perpendicular magnetic field. The actuation step in Fig. 12c, is carried out by considering a cantilever boundary condition for the $h$-MRE along with the mechanical and magnetic boundary conditions at the air boundary, so that

$$
\begin{aligned}
& \mathbf{u}_{\tau}=\mathbf{0}, \quad \forall \mathbf{X} \in \partial \mathcal{V}_{\mathrm{MRE}}^{\mathrm{Left}} \quad \text { and } \quad \mathbf{u}_{\tau}=\mathbf{0}, \quad \forall \mathbf{X} \in \partial \mathcal{V}_{\mathrm{Air}} \\
& \varphi_{\tau}=0, \quad \forall \mathbf{X} \in \partial \mathcal{V}_{\text {Air }}^{\text {Bottom }} \quad \text { and } \quad \varphi_{\tau}=-\frac{b_{2, \tau}^{\text {app }}}{\mu_{0}} L_{\mathrm{Air}} \quad \forall \mathbf{X} \in \partial \mathcal{V}_{\mathrm{Air}}^{\text {Top }},
\end{aligned}
$$

In addition, the temporal evolution for $b_{2}^{\text {app }}$ is shown in Fig. 12c.

It should be noted at this point that, the pre-magnetization step (Step-1) requires a considerably high applied magnetic field close to saturation, whereas, the actuation step (Step-2) only considers a maximum loading up to $50 \mathrm{mT}$.

The two important material parameters for the pre-magnetized $h$-MRE are the effective shear modulus $G$ of the composite and the effective remanent magnetization after the pre-magnetization step. The measured values for these two are provided by Zhao et al. (2019) to be $G=0.303 \mathrm{MPa}$ and $0.114 \mathrm{MA} / \mathrm{m}$, respectively. We thus set the particle volume fraction $c=0.177$ to obtain the aforementioned local remanent magnetization in $\mathcal{V}_{\mathrm{MRE}}$ after the pre-magnetization at $2 \mathrm{~T}$, while all the purely magnetic and coupling material parameters are kept identical to those provided in Table 2 . A straightforward calculation from $(4.2)_{1}$ with $G=0.303 \mathrm{MPa}$ and $c=0.177$ yields the matrix shear modulus $G_{\mathrm{m}} \approx 0.186$ $\mathrm{MPa}$ for ideally incompressible composites. The numerical simulations are conducted employing the constitutive model (4.9) with $G_{\mathrm{m}}=0.186 \mathrm{MPa}$ and $G_{\mathrm{m}}^{\prime}=500 G_{\mathrm{m}}$.

The contours of the magnitudes of $\mathbf{b}, \mathbf{h}$ and $\mathbf{m}$ fields along with their directions are shown in Fig. 13 at the beginning (a-c) and end (d-f) of Step-2. As observed in Fig. 13c, we obtain a uniformly pre-magnetized $h$-MRE after Step-1. The pre-magnetization fields are indicated with a superscript " 0 ", while the magnetic fields due to the Step-2 transverse magnetic loading are indicated only by the respective symbols. Notice that the local $\mathbf{h}$ field in the MRE structure remains small compared to the $\mathbf{b}$ and $\mathbf{m}$ fields, except at its left and right boundaries due to corner effects. Thus, one can approximate $\mathbf{b} \approx \mu_{0} \mathbf{m} \forall \mathbf{X} \in \mathcal{V}_{\mathrm{MRE}}$ for this specific case of uniformly pre-magnetized $h$-MREs. In fact, this approximation also seems to hold under applied actuation field $b_{2}^{\text {app }}=50 \mathrm{mT}$, which, in turn, leads to high deflection of the cantilever (see Fig. 13d-f). This property of exhibiting extreme mechanical deflection without changing its magnetic properties under applied small fields makes the slender $h$-MREs an ideal candidate for remote robotic actuation (Kim et al., 2018; Ren et al., 2019; Alapan et al., 2020).

The computed end-tip deflections of the pre-magnetized cantilever beams are then compared to the corresponding experimental measurements reported by Zhao et al. (2019) for two slenderness ratios, namely $r_{\text {asp }}=10$ and 17.5 . As shown in Fig. 14a, the agreement between the present simulations and the experiments is excellent. For further comparison, in Figs 14b,c, we show, respectively, the entire experimental and numerical deflected beam shapes for $r_{\text {asp }}=10$ at an applied magnetic field $b_{2}^{\text {app }}=25 \mathrm{mT}$. The agreement again is found to be good. A code developed by Mukherjee et al. (2021) based on ABAQUS/Standard reproducing the results in Figs 13 and 14 is available at https://www.doi.org/10.5281/zenodo.4588578. 
(a)

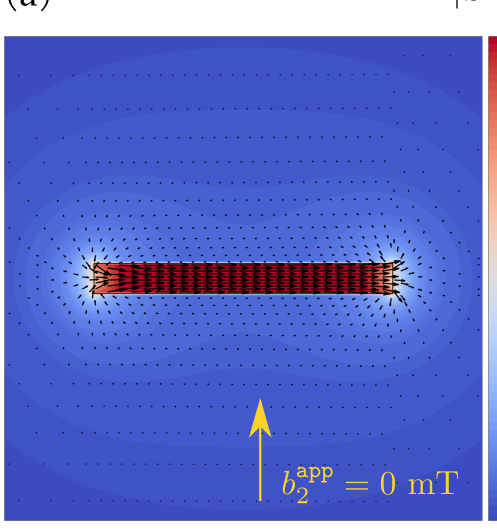

$\mathbf{b}^{0} \mid(\mathrm{T})$

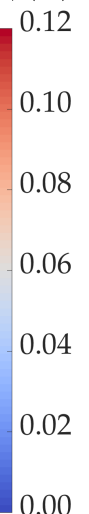

(d)

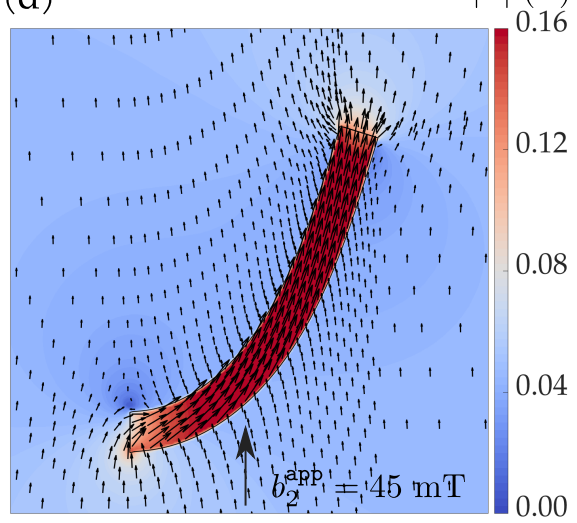

(b)

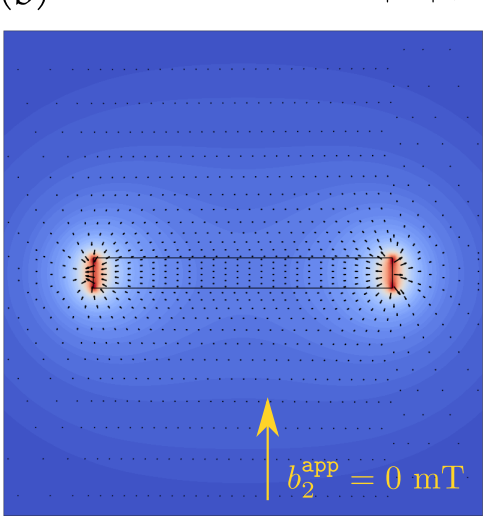

$\mathbf{h}^{0} \mid(\mathrm{MA} / \mathrm{m})$

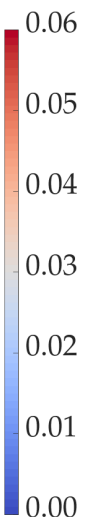

(e)

h| (MA/m)

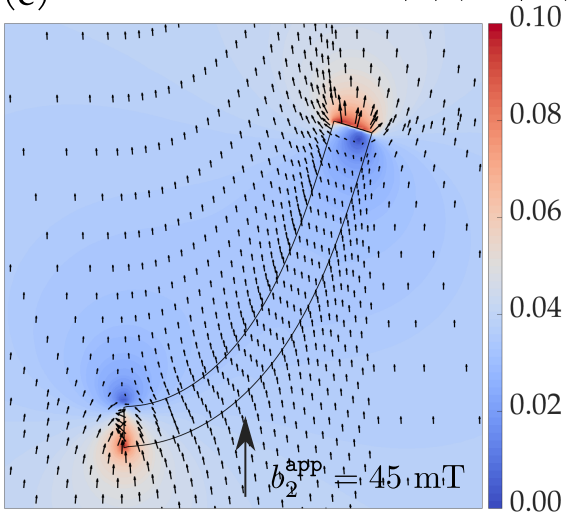

(c)

$\left|\mathbf{m}^{0}\right|(\mathrm{MA} / \mathrm{m})$

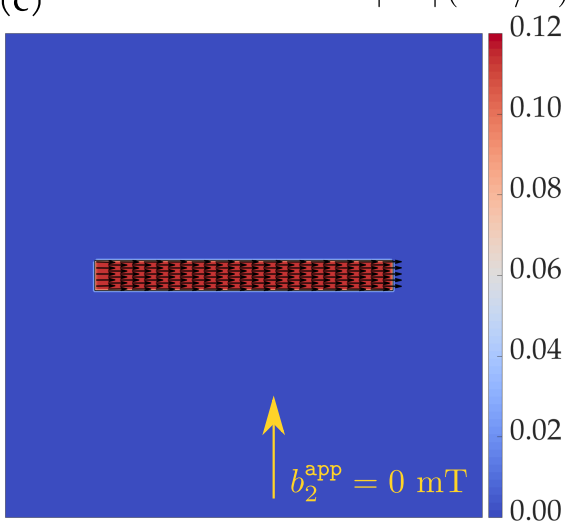

(f)

$|\mathbf{m}|(\mathrm{MA} / \mathrm{m})$

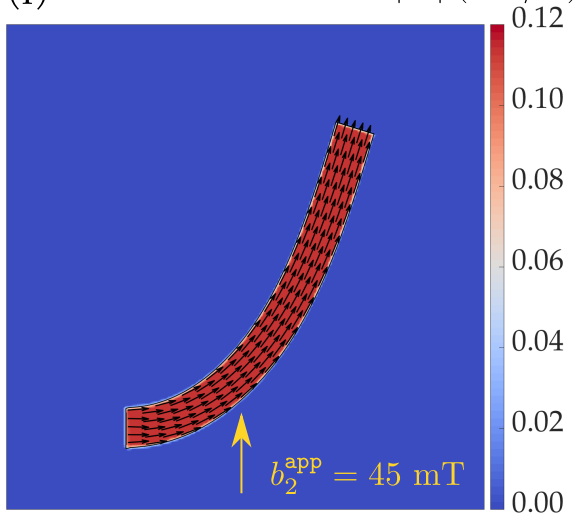

Figure 13: Residual magnetic (a) b, (b) $\mathbf{h}$ and (c) magnetization $\mathbf{m}$ fields in the $h$-MRE having an aspect ratio $r_{\text {asp }}=10$, along with magnetic self fields around the surrounding air after Step 1, i.e., the initial magnetization at $2 \mathrm{~T}$. The colored contours represent the magnitude of these fields, whereas the black arrows represent their directions. The lengths of these arrows denote the relative magnitude of the vectors. (d-f) Deflected pre-magnetized $h$-MRE under an applied transverse field of $45 \mathrm{mT}$ along $\mathbf{e}_{2}$. Magnitude and directions of the resulting (d) $\mathbf{b}$, (e) $\mathbf{h}$ and (f) $\mathbf{m}$ fields in the $h$-MRE and its surrounding air is shown by colored contours and black arrows, respectively.

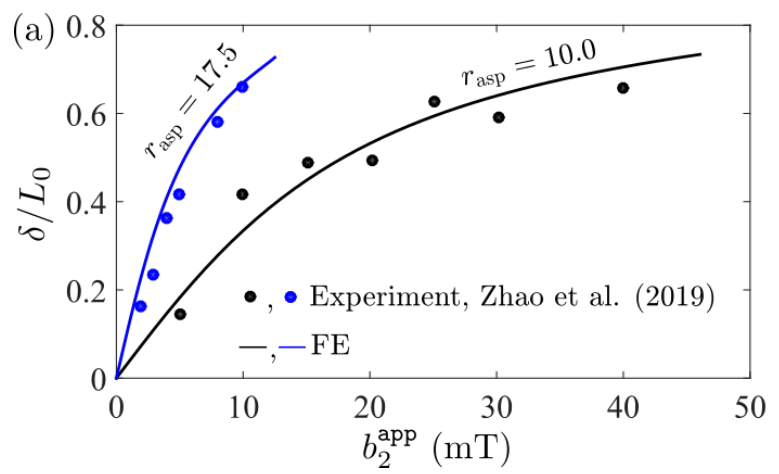

(b) Experiment

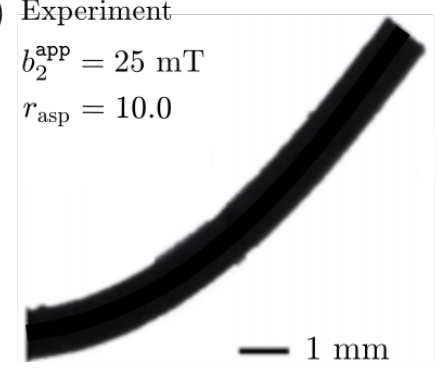

(c) $\mathrm{FE}$

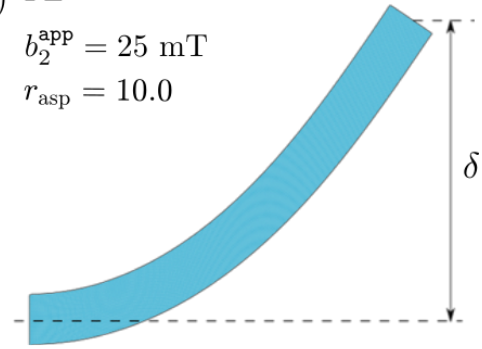

Figure 14: (a) Comparison between numerically computed and experimentally measured end-tip displacement of the pre-magnetized $h$-MREs (following Steps 1 and 2 as depicted in Fig. 12b and c) having two different aspect ratios $r_{\text {asp }}=10$ and 17.5 . (b) Experimentally captured deflected beam having $r_{\text {asp }}=10$ under $b_{2}^{\text {app }}=25 \mathrm{mT}$ and (c) numerically computed shape of the pre-magnetized beam of the same $r_{\text {asp }}$ under the same applied $b$-field.

\subsection{Deflection of non-uniformly pre-magnetized cantilever beams}

Non-uniformly pre-magnetized slender structures are not only of theoretical but also of practical interest. In a recent example of a locomotive application, Ren et al. (2019) fabricated jellyfish-like swimming robots having remotely-actuated flapping $h$-MRE structures. Motivated from the pre-magnetization process described in Ren et al. (2019), we consider here three idealized plane-strain geometries of slender $h$-MRE beams having $r_{\text {asp }}=10$. The three beams are pre-magnetized by fixing them to molds with different geometrical profiles (see Fig. 15), which evidently leads to substantially different pre-magnetization profiles. Subsequently, the pre-magnetization field is removed and the beams are released from the mold almost recovering their original straight shape (the resulting magnetostrictive effects are fairly small and do not change essentially the beam geometry). Finally, the beams are subjected to a small actuation field applied perpendicular to the long axis of the beam. 

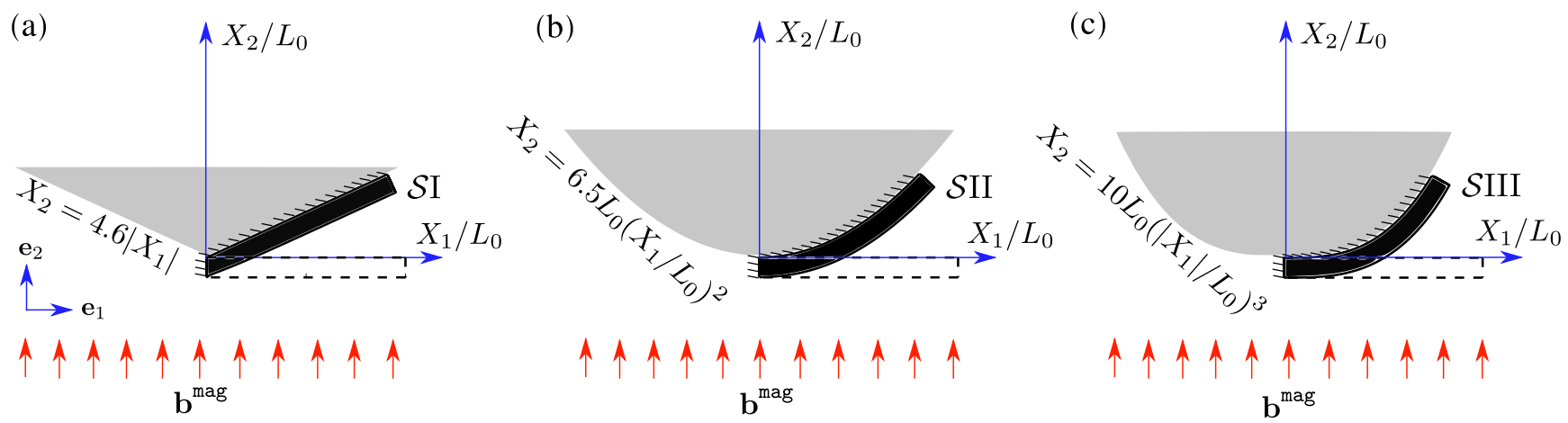

Figure 15: Pre-deformation geometries before the initial magnetization along $\mathbf{e}_{2}$ of three different samples of $h$-MREs, namely (a) $\mathcal{S}$ I, (b) $\mathcal{S}$ II and (c) $\mathcal{S}$ III, all having the same length $L_{0}$ and width $\mathrm{w}_{0}$ in their undeformed configurations. The pre-magnetizing field $\mathbf{b}^{\mathrm{mag}}$ direction is shown by red arrows, while its magnitude remains the same as in Fig. 12b. The pre-deformed profiles of the top boundary of the $h$-MREs are given by $X_{2}=4.6\left|X_{1}\right|, X_{2}=6.5 L_{0}\left(X_{1} / L_{0}\right)^{2}$ and $X_{2}=10 L_{0}\left(\left|X_{1}\right| / L_{0}\right)^{3}$ for the samples $\mathcal{S}$ I, $\mathcal{S}$ II and $\mathcal{S}$ III, respectively.

(a)

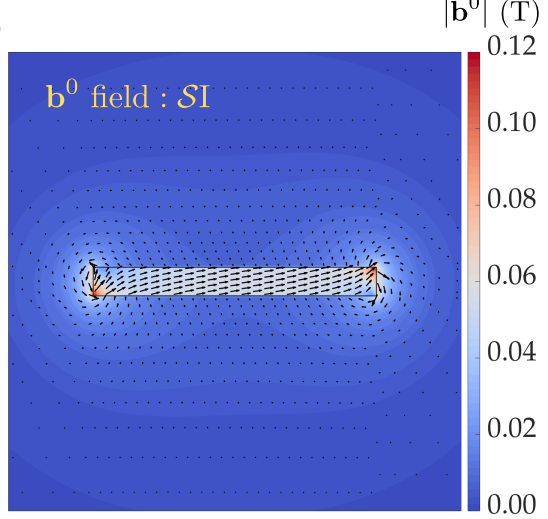

(d)

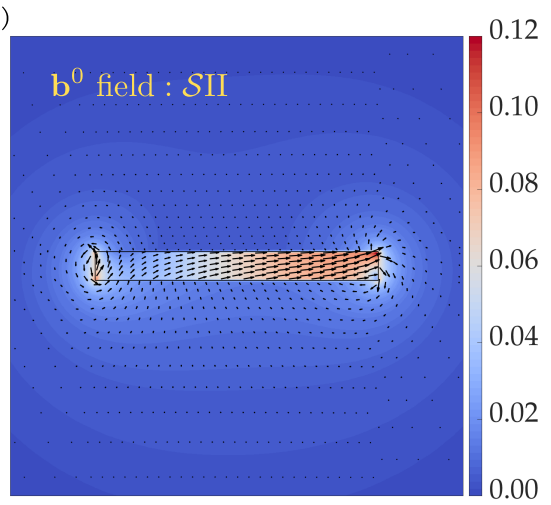

$(\mathrm{g})$

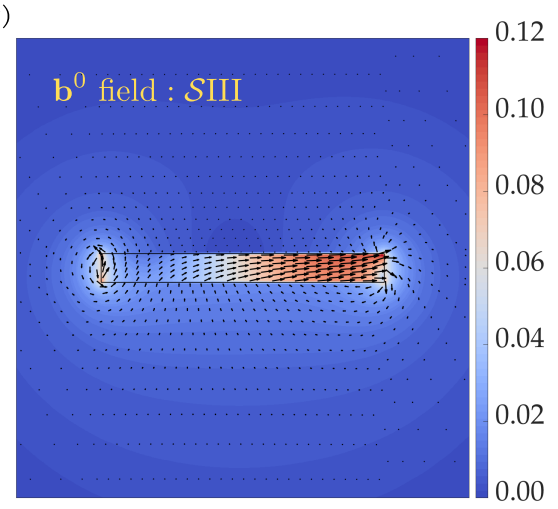

(b)

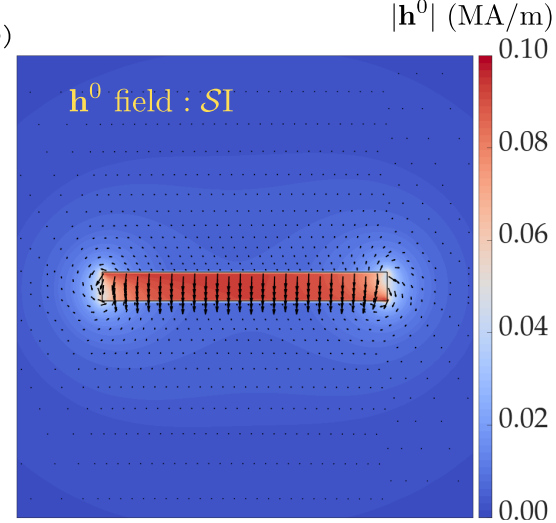

(e)

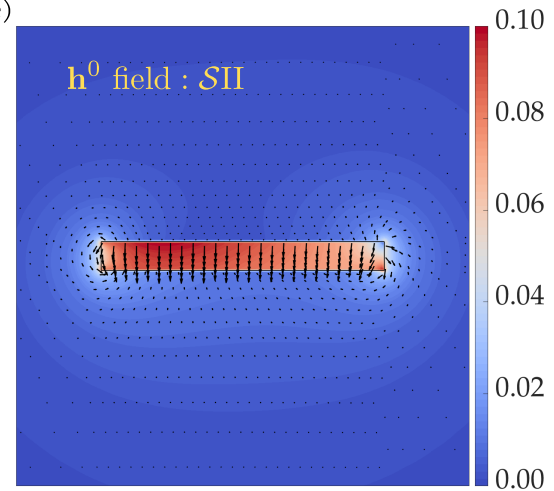

(h)

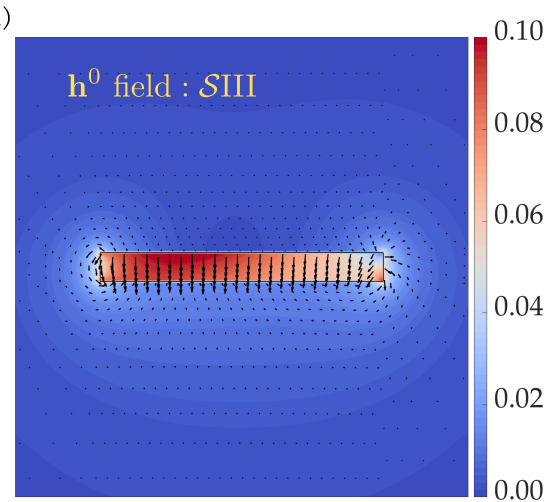

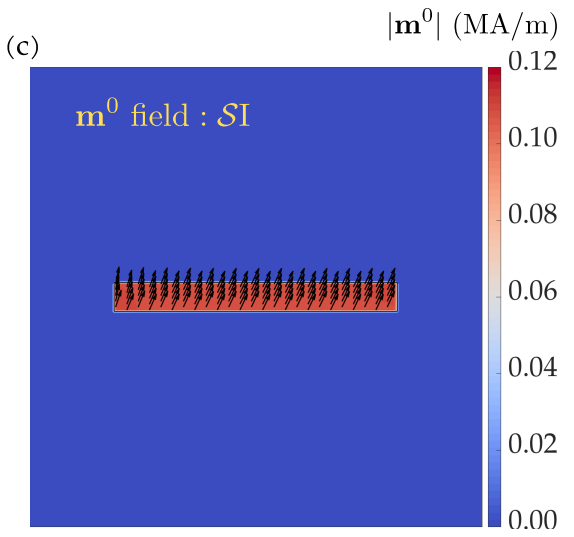

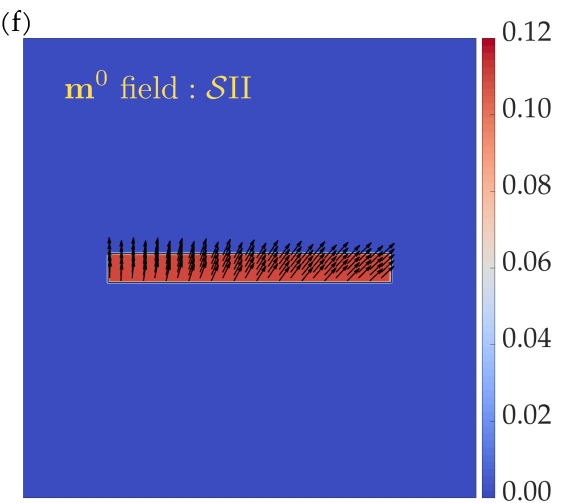

(i)

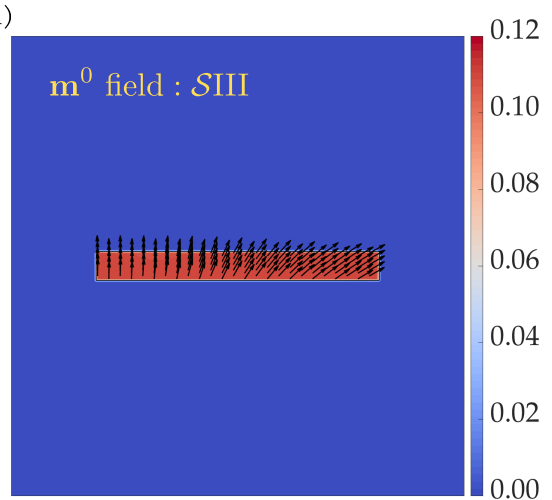

Figure 16: Magnitude and directions of residual $\mathbf{b}, \mathbf{h}$ and $\mathbf{m}$ fields in (a-c) $\mathcal{S}$, (d-f) $\mathcal{S}$ II and (g-i) $\mathcal{S}$ III after the pre-magnetization of the deformed samples along $\mathbf{e}_{2}$ (see schematic diagrams in Fig. 15), following a loading path qualitatively similar to Fig. 12b, but having an amplitude of $2 \mathrm{~T}$ and after the release of their pre-deformation. The colored contours represent the magnitude of the vector fields, while the black arrows represent their directions. 

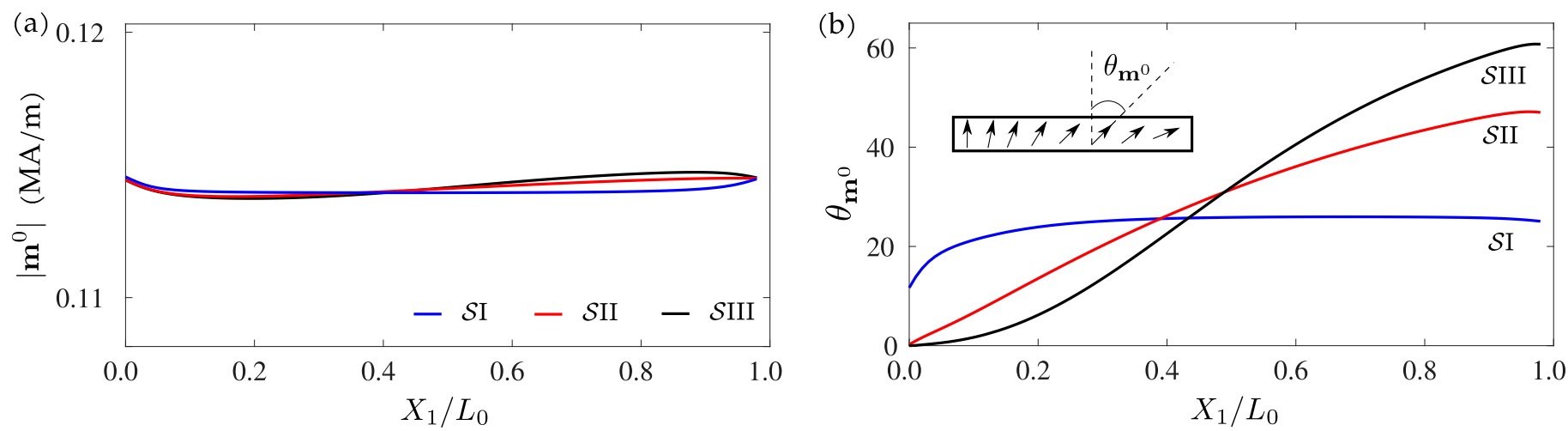

Figure 17: Variation of the residual magnetization $\mathbf{m}^{0}$, induced by the magnetization of the pre-deformed $h$-MREs, along its length after the pre-deformation is released. The variation of (a) magnitude and (b) direction of the $\mathbf{m}^{0}$ vector is shown along the centerline of three differently pre-deformed $h$-MREs, namely, $\mathcal{S}$, $\mathcal{S}$ II and $\mathcal{S}$ III.

(a)
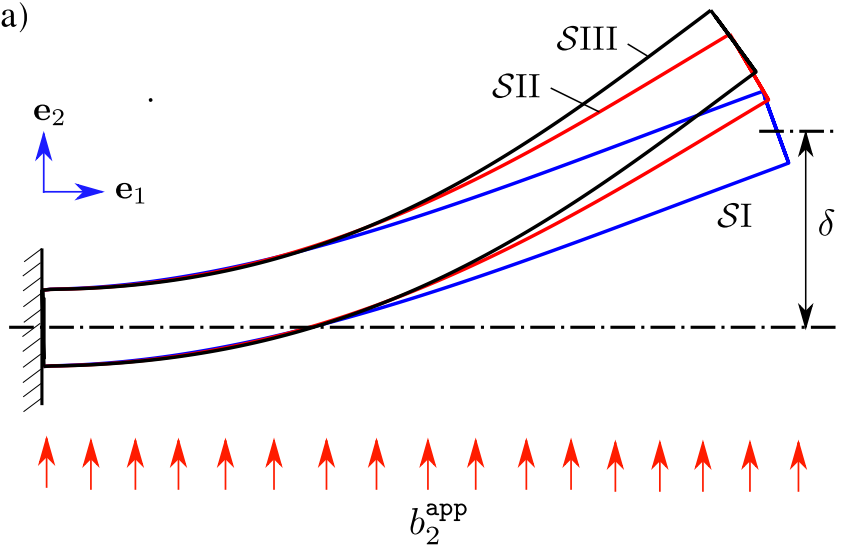

(b)

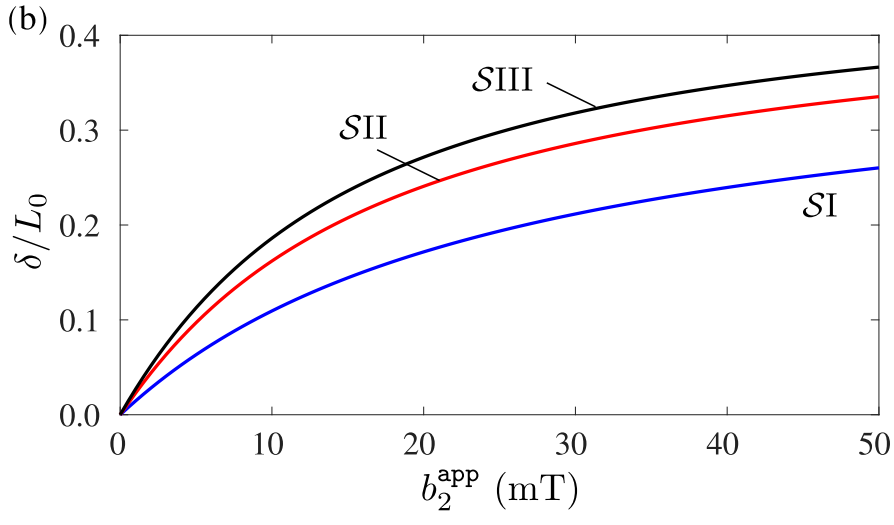

Figure 18: Deflection of non-uniformly magnetized $h$-MREs $\mathcal{S}$, $\mathcal{S}$ II and $\mathcal{S}$ III under applied field $b_{2}^{\text {app }}$ having amplitude shown in Fig. $12 \mathrm{c}$ and direction along $\mathbf{e}_{2}$. (a) Deflected shapes of $\mathcal{S}$ I, $\mathcal{S}$ II and $\mathcal{S}$ III under applied $b_{2}^{\text {app }}=50$ mT. (b) Evolutions of the normalized end-tip deflections $\delta / L_{0}$ of $\mathcal{S}$ I, $\mathcal{S}$ II and $\mathcal{S}$ III with $b_{2}^{\text {app }}$.

To solve this problem numerically, we employ the same principle, mesh and material parameters used in the previous section (Fig. 12). In turn, the solution process consists of the following four steps:

- Step-1: Profiling of the beam. The first step, now, consists in the pre-deformation, where prescribed displacements $\mathbf{u}_{\tau}=\mathbf{u}_{\tau}^{\text {Top }}$ are applied on the boundary $\partial \mathcal{V}_{\mathrm{MRE}}^{\text {Top }}$, while $\mathbf{u}_{\tau}=\mathbf{0}$ is maintained for all $\mathbf{X} \in \partial \mathcal{V}_{\mathrm{MRE}}^{\mathrm{Left}}$. These displacements are applied via the "DISP" subroutine of Abaqus, in such a way that the top boundary of the $h$-MREs adheres to (a) a linear, (b) a quadratic and (c) a cubic mold profile, whose specific functional forms are indicated in Fig. $15^{11}$.

- Step-2: Pre-magnetization of the beam. In this step, we apply a pre-magnetizing field $\mathbf{b}^{\text {mag }}=b_{2}^{\text {mag }} \mathbf{e}_{2}$ similar to that prescribed in (7.9). The temporal variation of the applied $b_{2}^{\mathrm{mag}}$ is considered similar to that in Fig. 12b.

- Step-3: Release of the beam. In this step, the applied pre-displacement is released, i.e., $\mathbf{u}^{\text {Top }}$ is decreased to zero allowing the beam to recover its (almost) original form.

- Step-4: Actuation via a perpendicular magnetic field. In this step, we apply exactly the same boundary conditions as in (7.8) and (7.9) with the same magnetic loading described by Fig. 12c.

The contours of the $\left|\mathbf{b}^{0}\right|,\left|\mathbf{h}^{0}\right|$ and $\left|\mathbf{m}^{0}\right|$ fields along with their directions in the beginning of Step- 4 are shown in Fig. 16 for the pre-magnetization geometries $\mathcal{S}$ I (a-c), $\mathcal{S}$ II (d-f) and $\mathcal{S} I I I$ (g-i). Again, the superscript "0" indicates the induced magnetic fields after the pre-magnetization and relaxation process. Figures 16c,f,i show that the non-uniformity of the pre-magnetization field in the beam increases with the increasing pre-bending of the structure. Thus, $\mathcal{S} I I I$ exhibits a significant non-uniformity in the direction of $\mathbf{m}^{0}$, while its magnitude remains almost the same throughout the beam.

Similarly, the distributions of the $\mathbf{b}^{0}$ and $\mathbf{h}^{0}$ fields in the beams are also not uniform, especially, for the premagnetization profiles $\mathcal{S}$ II and $\mathcal{S} I I I$. Moreover, the local $\mathbf{h}^{0}$ fields are of the same order as the $\mathbf{m}^{0}$ fields, thus, contrasting the previous case of uniformly magnetized beams. As a result, in this case of non-uniformly magnetized beams,

\footnotetext{
${ }^{11}$ The "DISP" subroutine of Abaqus applies displacement in terms of the current coordinate $\mathbf{x}$. The specific displacement functions employed in the numerical computations are (i) $u_{1}^{\text {Top }}=-0.10 x_{1}, u_{2}^{\text {Top }}=0.46 x_{1}$, (ii) $u_{1}^{\text {Top }}=-0.30 L_{0}\left(x_{1} / L_{0}\right)^{2}, u_{2}^{\text {Top }}=0.65 L_{0}\left(x_{1} / L_{0}\right)^{2}$ and (iii) $u_{1}^{\text {Top }}=-0.62 L_{0}\left(x_{1} / L_{0}\right)^{3}, u_{2}^{\text {Top }}=L_{0}\left(x_{1} / L_{0}\right)^{3}$ for $\mathcal{S}$ I, $\mathcal{S}$ II and $\mathcal{S}$ III, respectively. These displacements are chosen heuristically that roughly ensure an un-stretched centerline of the beam, having length $L_{0}$ in the pre-deformed configurations, shown in Fig. 15 . Although more sophisticated means of imposing such a condition may be possible, those methods are not employed here for simplicity given that we are studying an idealized problem.
} 
approximating the local $\mathbf{b}^{0}$ fields simply by $\mathbf{b}^{0}=\mu_{0} \mathbf{m}^{0}$ may lead to significant errors and such a process should at least be rigorously verified with corresponding full field simulations including the surrounding air as done in the present study.

From a more quantitative point, we show in Fig. 17 the non-uniformity of (a) the magnitude of $\mathbf{m}^{0}$ and (b) the orientation angle of $\mathbf{m}^{0}$ with respect to the vertical axis $\mathbf{e}_{2}$ along the centerline of the beam for all three pre-magnetization cases $\mathcal{S}$ I, $\mathcal{S}$ II and $\mathcal{S}$ III. Evidently, the magnitude of $\mathbf{m}^{0}$ remains almost the same and fairly uniform for all the three cases. By contrast, the orientation of $\mathbf{m}^{0}$ varies significantly, with the $\mathcal{S} I I I$ exhibiting the more heterogeneous profile.

As a result of this highly non-uniform pre-magnetization profile, the actuated deflections of the beams are different for the same externally applied $\mathbf{b}^{\text {app }}$. In particular, Fig. $18 \mathrm{a}$ shows the deflected shapes of these beams at $b_{2}^{\text {app }}=50 \mathrm{mT}$ and Fig. 18b reports the corresponding evolution of their end-tip deflection $\delta$ as a function of $b_{2}^{\text {app }}$. We observe that the beam $\mathcal{S}$ III exhibits the maximum deflection. This implies obviously that different pre-magnetization and loading patterns may lead to a variety of deflection responses of beams and slender structures more generally. This mechanism is exploited ingeniously in Ren et al. (2019) to actuate asymmetric bending responses at different parts of a soft robot remotely via a magnetic field leading to locomotion and swimming.

\section{Concluding remarks}

In this paper, we propose an explicit, analytical constitutive model for isotropic incompressible (and quasi-incompressible) $h$-MREs comprising hard-magnetic (of any type), mechanically-rigid particles embedded in elastomeric matrix. The model involves explicitly the dependence on the particle volume fraction as well as the individual properties of the constituents. Additionally, as a mere result of the dissipative magnetic response of the particles, it is capable of delivering a macroscopic coupled dissipative response upon application of arbitrary proportional or non-proportional (e.g. cyclic) magnetic or mechanical loading conditions.

The model is constructed following rigorous mathematical and physics principles. In particular, we ensure thermodynamic consistency of the model by deriving the constitutive relations from the localized Clausius-Duhem inequalities and by employing the framework of generalized standard materials, which yields the evolution laws for the remanent internal variables. The objectivity and material symmetry properties of the isotropic $h$-MREs are obtained by proposing the energetic and dissipation potentials in terms of suitable invariants involving both energetic and remanent parts.

Specifically, the present model uses a single (vector-valued) internal variable, the remanent magnetic $h$-field $\mathcal{H}^{r}$, which is defined in the intermediate stretch-free configuration. This choice is motivated by rigorous full field RVE simulations, which show that the resulting current effective remanent $h$-field and consequently the current magnetization are completely unaffected by the macroscopic stretch. This further implies that the amplitude of the current magnetization is independent of changes in the macroscopic deformation gradient. In turn, the current magnetization orientation may change depending on the applied loads and its rotation is a complex function of the underlying particle rotations. In the present study, we assume an affine particle rotation model that is valid for practically relevant $h$-MREs having a moderately soft polymeric matrix (with shear modulus $G_{\mathrm{m}} \geq 0.15 \mathrm{MPa}$, although in practical applications the model can be used even for softer matrices but with caution). In those cases, identifying the rotation of the remanent $h$-field with the macroscopic rotation tensor leads to an explicit model, while it delivers sufficiently accurate estimates for both the effective magnetic and mechanical fields under complex loading conditions (for instance, non-aligned, cyclic magnetic loads with the pre-magnetization of the RVE).

The proposed modeling framework inherently takes care of the magnetic body force and body torque-like terms in a magneto-active solid by incorporating their effects in the total stress. Thus, no additional body force/torque-like terms are required to be incorporated in the local linear/angular momentum balance equations. The total Cauchy stress remains symmetric in the proposed modeling framework. In particular, the Helmholtz free energy associated with the proposed macroscopic models of the $h$-MREs is considered to be the sum of three distinct contributions, namely the mechanical, magnetic and coupling free energies. Only three additional modeling parameters are introduced in the macroscopic model, which are then estimated by calibrating the model predictions with the numerical homogenization results.

As a result of this construction, the proposed model admits a number of useful limiting cases already obtained in the literature for $s$-MREs or the response of the individual constituents. Specifically, in the limit of a soft magnetic response, i.e., $b_{\mathrm{p}}^{c} \rightarrow 0$, the stress measures reduce to those defined by Kankanala and Triantafyllidis (2004). Moreover the resulting effective model response reproduces exactly the response from the analytical homogenization model of Lefèvre et al. (2017). Secondly, in the limit of $c=1$, the proposed $\boldsymbol{\sigma}$ yields the classical definition of the total $\boldsymbol{\sigma}$ by Robinson (1975) in the context of metallic permanent magnets.

The assessment of the proposed constitutive model is carried out in two steps. First, the model response is compared with full-field numerical RVE homogenization results and second, the model is employed in a numerical boundary value problem in order to probe its performance to the experimental measurements of Zhao et al. (2019).

Our numerical realizations of both, the microscopic fields in the RVE and the macroscopic fields in the macroscopic BVPs benefit from the definition of suitable (incremental) variational principles. In particular, an incremental potential is derived following the work of Miehe et al. $(2002,2011)$ by appropriately reformulating it in the present context of dissipative magneto-elasticity. This incremental potential is then used to frame the incremental variational principles for both, microscopic RVE computations and macroscopic structural computations. 
The model responses both under proportional and non-proportional loading paths show comprehensive agreements with the full-field numerical homogenization estimates. Specifically, the model responses under aligned magnetic and mechanical loadings of a pre-magnetized $h$-MRE are in excellent agreement with the numerical homogenization results. In turn, non-proportional magnetic loading gradually leads to a less accurate comparison mainly due to non-affine particle rotations in the mechanically softer $h$-MREs (e.g. $G_{\mathrm{m}}<0.3 \mathrm{MPa}$ ). In this regard, it is noted that the non-affine particle rotations control the magnetization/demagnetization responses of the softer, gel-like $h$-MREs having $G_{\mathrm{m}} \sim 0.01-0.1 \mathrm{MPa}$ (Linke et al., 2016; Kalina et al., 2017). Of course, the proposed affine rotation model cannot be applied readily to model such highly compliant composites. Indeed, further refinements to the proposed framework are needed in those cases. Nonetheless, it is noted that most of the practical applications of the $h$-MREs to-date consider relatively stiffer PDMS matrix having $G_{\mathrm{m}} \sim 0.15-1.5 \mathrm{MPa}$. Moreover, such $h$-MREs, once permanently magnetized, are typically subjected to high mechanical but only very low magnetic fields (Kim et al., 2018; Zhao et al., 2019; Ren et al., 2019; Alapan et al., 2020). Contextually, in the aforementioned loading regime, the proposed affine rotation model is observed to predict the microstructure response sufficiently well. Hence, it can be readily employed to solve many magneto-mechanical boundary value problems of practical interest even for softer materials. Moreover, the proposed model involves fairly simple terms and is versatile enough to allow for ad-hoc calibration via the coupling parameter $\beta$ or even via the homogenized parameters $\chi^{e}, \chi^{r}, m_{s}$ and $b^{c}$, which can be fitted independently and without any reference to the particle magnetic properties and volume fraction.

In this regard, the proposed model is applied to solve for the deflection of pre-magnetized $h$-MRE cantilever beams, subjected to remotely applied transverse magnetic fields. The model prediction is found to be in excellent agreement with the experimentally measured end-tip deflections of the $h$-MREs. We further apply the model in non-uniformly pre-magnetized beams. Therein, we find that even though the pre-magnetized $h$-MRE structures are not subjected to high magnetic fields, the presence of the surrounding air and the pre-magnetization process itself is crucial to estimate the local pre-magnetic $\mathbf{b}, \mathbf{h}$ and $\mathbf{m}$ fields in the structure. In this context, we also point the reader towards the recent work of Alapan et al. (2020), where the measured stray $b$-fields around the $h$-MRE structures exhibit significant non-uniformity. Hence, the proposed fully hysteretic constitutive framework having an evolving remanent field finds direct relevance in the modeling of non-uniformly pre-magnetized structures, even though they are not exposed to high magnitude of actuation magnetic fields after their pre-magnetization.

Finally, the applicability of the proposed model does not remain confined within pre-magnetized structures under applied non-aligned magnetic fields. Various pre-magnetized structures are employed in sensing devices, where the perturbations in its magnetic self-field is measured (Kaidarova et al., 2018; Sitti and Wiersma, 2020). Further works on the application of the proposed model in various sensing device-related BVPs is underway. Last but not least, the present model can be further simplified in the case of small actuating fields around a pre-magnetized state. Such an effort is underway and will be presented elsewhere.

\section{Acknowledgements}

The authors would like to acknowledge support from the European Research Council (ERC) under the European Union's Horizon 2020 research and innovation program (grant agreement No 636903 - MAGNETO). The computational part of this work was also supported by the ANR, France under contract number ANR-10-EQPX-37. The code developed for the numerical simulations in this work is available at https://www.doi.org/10.5281/zenodo.4588578.

\section{Appendix A. Eulerian formulation of the thermodynamic framework}

The Lagrangian and Eulerian forms of the Clausius-Duhem inequalities can be obtained via performing localization of the global entropy imbalance equations at the reference and the current configurations, respectively (Kankanala and Triantafyllidis, 2004; Dorfmann and Ogden, 2004). We point out that in the Eulerian description below, $\mathbf{h}$ and $\mathbf{h}^{r}$ are now the independent magnetic and internal variables, respectively.

We start by recovering the localized Eulerian form of the Clausius-Duhem inequality from its Lagrangian counterpart $(3.3)$

$$
\mathbf{S}: \dot{\mathbf{F}}-\mathbf{B} \cdot \dot{\mathbf{H}}-\dot{W} \geq 0 .
$$

Substituting $\mathbf{S}=J \boldsymbol{\sigma} \mathbf{F}^{-T}$ along with $\mathbf{B}=J \mathbf{F}^{-1} \mathbf{b}$ and $\mathbf{H}=\mathbf{F}^{T} \mathbf{h}$ into the last equation we obtain

$$
J \boldsymbol{\sigma}: \dot{\mathbf{F}} \mathbf{F}^{-1}-J \mathbf{F}^{-1} \mathbf{b} \cdot \overline{\mathbf{F}^{\mathbf{T}} \mathbf{h}}-\dot{w} \geq 0
$$

Here, $w\left(\mathbb{B}, \mathbf{h}, \mathbf{h}^{r}\right)=W\left(\mathbb{C}, \mathbf{H}, \boldsymbol{\mathcal { H }}^{r}\right)$. Since the stretch rate $\boldsymbol{l}$ is defined via $\boldsymbol{l}=\dot{\mathbf{F}} \mathbf{F}^{-1}$, the last equation upon algebraic manipulations leads to

$$
J \boldsymbol{\sigma}: \boldsymbol{l}-J(\mathbf{h} \otimes \mathbf{b}): \boldsymbol{l}-J \mathbf{b} \cdot \dot{\mathbf{h}}-\dot{w} \geq 0 .
$$

Expanding subsequently the current potential $w$, so that $w=\rho_{0} \psi-J\left(\mu_{0} / 2\right) \mathbf{h} \cdot \mathbf{h}$ in the last expression we rephrase it to be

$$
J \boldsymbol{\sigma}: \boldsymbol{l}-J(\mathbf{h} \otimes \mathbf{b}): \boldsymbol{l}-J \mathbf{b} \cdot \dot{\mathbf{h}}-\rho_{0} \dot{\psi}+\frac{\mu_{0}}{2} J|\mathbf{h}|^{2} \boldsymbol{l}+\mu_{0} J \mathbf{h} \cdot \dot{\mathbf{h}} \geq 0
$$


Finally, collecting the coefficients of $\boldsymbol{l}$ and $\dot{\mathbf{h}}$ in the above we obtain

$$
J\left(\boldsymbol{\sigma}-\mathbf{h} \otimes \mathbf{b}+\frac{\mu_{0}}{2}|\mathbf{h}|^{2} \mathbf{I}\right): \boldsymbol{l}-\mu_{0} J \mathbf{m} \cdot \dot{\mathbf{h}}-\rho_{0} \dot{\psi} \geq 0 .
$$

Expanding subsequently the material rate of the Helmholtz free energy $\dot{\psi}\left(\mathbb{B}, \mathbf{h}, \mathbf{h}^{r}\right)$ of (A.5) in terms of its arguments (see also Remark 1), we obtain

$$
J\left(\boldsymbol{\sigma}-\mathbf{h} \otimes \mathbf{b}+\frac{\mu_{0}}{2}|\mathbf{h}|^{2} \mathbf{I}\right): \boldsymbol{l}-\mu_{0} J \mathbf{m} \cdot \dot{\mathbf{h}}-\rho_{0} \frac{\partial \psi}{\partial \mathbb{B}}: \dot{\mathbb{B}}-\rho_{0} \frac{\partial \psi}{\partial \mathbf{h}} \cdot \dot{\mathbf{h}}-\rho_{0} \frac{\partial \psi}{\partial \mathbf{h}^{r}} \cdot \dot{\mathbf{h}}^{r} \geq 0,
$$

which upon rearrangement finally reads

$$
\begin{aligned}
& J\left[\boldsymbol{\sigma}-\frac{2 \rho_{0}}{J} \frac{\partial \psi}{\partial \mathbb{B}} \mathbb{B}-\left(\mathbf{h} \otimes \mathbf{b}-\frac{\mu_{0}}{2}|\mathbf{h}|^{2} \mathbf{I}\right)-\frac{\rho_{0}}{J}\left\{\left(\frac{\partial \psi}{\partial \mathbf{h}^{r}} \otimes \mathbf{R}^{T} \mathbf{h}^{r}\right): \frac{\partial \mathbf{R}}{\partial \mathbf{F}}\right\} \mathbf{F}^{T}\right]: \boldsymbol{l} \\
- & J\left[\mathbf{m}+\frac{\rho_{0}}{J} \frac{\partial \psi}{\partial \mathbf{h}}\right] \cdot \dot{\mathbf{h}}-\rho_{0} \frac{\partial \psi}{\partial \mathbf{h}^{r}} \cdot \overrightarrow{\mathbf{h}}^{r} \geq 0,
\end{aligned}
$$

where $\overrightarrow{\mathbf{h}}^{r}=\dot{\mathbf{h}}^{r}-\boldsymbol{\Omega} \mathbf{h}^{r}$ is the objective Green-Naghdi rate of the remanent $h$-field with $\boldsymbol{\Omega}=\dot{\mathbf{R}} \mathbf{R}^{T}$ is the mechanical spin tensor (Green and Naghdi, 1965) ${ }^{12}$. Notably, the Green-Naghdi rate of the internal variable in (A.7) ensures the energy dissipation to remain independent of the mechanical spin $\boldsymbol{\Omega}$. In other words, the Green-Naghdi rate of $\mathbf{h}^{r}$ ensures that, in the absence of external magnetic fields, a rigid rotation of a permanently magnetized $h$-MRE does not add to any energy dissipation.

From Remark 1 we know that the sum in the first square bracket of (A.7) is symmetric. This enables us to replace the velocity gradient $\boldsymbol{l}$ by the Eulerian rate of deformation tensor $\boldsymbol{d}=\operatorname{sym}[\boldsymbol{l}]$, which lets us arrive at

$$
\begin{aligned}
& J\left[\boldsymbol{\sigma}-\frac{2 \rho_{0}}{J} \frac{\partial \psi}{\partial \mathbb{B}} \mathbb{B}-\left(\mathbf{h} \otimes \mathbf{b}-\frac{\mu_{0}}{2}|\mathbf{h}|^{2} \mathbf{I}\right)-\frac{\rho_{0}}{J}\left\{\left(\frac{\partial \psi}{\partial \mathbf{h}^{r}} \otimes \mathbf{R}^{T} \mathbf{h}^{r}\right): \frac{\partial \mathbf{R}}{\partial \mathbf{F}}\right\} \mathbf{F}^{T}\right]: \boldsymbol{d} \\
&-J\left[\mathbf{m}+\frac{\rho_{0}}{J} \frac{\partial \psi}{\partial \mathbf{h}}\right] \cdot \dot{\mathbf{h}}-\rho_{0} \frac{\partial \psi}{\partial \mathbf{h}^{r}} \cdot \dot{\mathbf{h}}^{r} \geq 0,
\end{aligned}
$$

where $\boldsymbol{d}=\operatorname{sym}[\boldsymbol{l}]$ is the symmetric part of the mechanical deformation rate. Owing to the arbitrariness ${ }^{13}$ of $\boldsymbol{d}$ and $\dot{\mathbf{h}}$, the standard arguments of the Coleman-Noll-Gurtin framework leads to the constitutive relations for the total Cauchy stress and the current $b$-field, such that

$$
\boldsymbol{\sigma}=\frac{2 \rho_{0}}{J} \frac{\partial \psi}{\partial \mathbb{B}} \mathbb{B}+\left(\mathbf{h} \otimes \mathbf{b}-\frac{\mu_{0}}{2}|\mathbf{h}|^{2} \mathbf{I}\right)+\frac{\rho_{0}}{J}\left\{\left(\frac{\partial \psi}{\partial \mathbf{h}^{r}} \otimes \mathbf{R}^{T} \mathbf{h}^{r}\right): \frac{\partial \mathbf{R}}{\partial \mathbf{F}}\right\} \mathbf{F}^{T}
$$

and

$$
\mathbf{m}=-\frac{\rho_{0}}{J} \frac{\partial \psi}{\partial \mathbf{h}} \quad \Rightarrow \quad \mathbf{b}=-\frac{1}{J} \frac{\partial w}{\partial \mathbf{h}}
$$

respectively, where the latter is obtained via the relation $w\left(\mathbb{B}, \mathbf{h}, \mathbf{h}^{r}\right)=\rho_{0} \psi\left(\mathbb{B}, \mathbf{h}, \mathbf{h}^{r}\right)-\left(\mu_{0} / 2\right) J \mathbf{h} \cdot \mathbf{h}$. Of course, the expression of total $\boldsymbol{\sigma}$ in terms of the mechanical, Maxwell and remanent parts remains identical to (3.8). Straightforward algebraic manipulations after substituting the fourth order tensor $\partial \mathbf{R} / \partial \mathbf{F}$, given by Chen and Wheeler (1993) into (A.9) leads to the expression for $\sigma$ given by

$$
\boldsymbol{\sigma}=\frac{2 \rho_{0}}{J} \frac{\partial \psi}{\partial \mathbb{B}} \mathbb{B}+\left(\mathbf{h} \otimes \mathbf{b}-\frac{\mu_{0}}{2}|\mathbf{h}|^{2} \mathbf{I}\right)+\frac{2}{J \operatorname{det} \mathbf{Z}} \mathbf{Z} \operatorname{skw}\left(\mathbf{h}^{r} \otimes \mathbf{b}^{r}\right) \mathbf{V Z}
$$

where $\mathbf{V}$ and $\mathbf{Z}$ are given as

$$
\mathbf{V}=\mathbf{F R}^{-1} \quad \text { and } \quad \mathbf{Z}=\operatorname{tr}[\mathbf{V}] \mathbf{1}-\mathbf{V}
$$

respectively. Subsequently, the Eulerian dissipation inequality reads

$$
\mathbf{b}^{r} \cdot \hat{\mathbf{h}}^{r} \geq 0, \quad \text { with } \quad \mathbf{b}^{r}=-\rho_{0} \frac{\partial \psi}{\partial \mathbf{h}^{r}}=-\frac{\partial w}{\partial \mathbf{h}^{r}}
$$

\footnotetext{
${ }^{12}$ The original work of Green and Naghdi (1965) propose objective rates in the context of mechanics. Nonetheless, the vectorial representation of the Green-Naghdi rate is referred in this paper.

${ }^{13}$ The current stretch rate $\boldsymbol{d}$ is independent of the spin $\boldsymbol{\Omega}=\dot{\mathbf{R}} \mathbf{R}^{T}$. Hence, the Green-Naghdi rate $\overrightarrow{\mathbf{h}}^{r}$, which is a function of the spin tensor, remains independent of $\boldsymbol{d}$.
} 
where $\mathbf{b}^{r}$ is the remanent $b$-field that is the energetic work conjugate of $\mathbf{h}^{r}$. Furthermore, the generalized standard material relation in the current configuration is given in terms of $w\left(\mathbb{B}, \mathbf{h}, \mathbf{h}^{r}\right)$ and $\mathscr{D}\left(\mathbf{h}^{r}\right)$, such that (Halphen and Son Nguyen, 1975)

$$
\frac{\partial w}{\partial \mathbf{h}^{r}}+\frac{\partial \mathscr{D}}{\partial \dot{\mathbf{h}}^{r}}=0 \quad \text { and } \quad \mathbf{b}^{r}=\frac{\partial \mathscr{D}}{\partial \dot{\mathbf{h}}^{r}} .
$$

Since the dissipation potential $\mathscr{D}\left(\overrightarrow{\mathbf{h}}^{r}\right)$ is defined in terms of the objective Green-Naghdi rate, we can rephrase the derivative of $\mathscr{D}$ with respect to $\dot{\mathbf{h}}^{r}$ in the last equation in terms of its derivative with respect to $\mathbf{h}^{r}$ by applying the chain rule, such that

$$
\frac{\partial w}{\partial \mathbf{h}^{r}}+\frac{\partial \mathscr{D}}{\partial \mathbf{h}^{r}}=0 \quad \Longrightarrow \quad \mathbf{b}^{r}=\frac{\partial \mathscr{D}}{\partial \mathbf{h}^{r}} .
$$

Concerning material frame indifference and isotropy we remark that all invariants introduced in Section 3.3 can be expressed equivalently in terms of $\mathbb{B}, \mathbf{h}$ and $\mathbf{h}^{r}$. As a consequence, the energy density and the dissipation potential of the Eulerian formulation automatically fulfill material frame indifference and isotropy when internally represented in terms of these invariants.

\section{Appendix B. Derivatives of $I_{4}^{e r}$ and $I_{5}^{e r}$ with respect to $\mathbb{C}$}

Computation of the first Piola-Kirchhoff stress in a Lagrangian setting from the constitutive relation (3.5) is performed via finding the derivative of (4.1) with respect to $\mathbb{C}$. The computation of $\mathbf{S}$ is involved with computing the derivatives of the invariants defined in the equations (3.13), (3.14) and (3.15) with respect to $\mathbb{C}$. In this regard, it is noted from $(3.13),(3.14)$ and (3.15) that the derivatives of all the invariants therein with respect to $\mathbb{C}$ are straightforward except the mixed invariants $I_{4}^{e r}$ and $I_{5}^{e r}$, which are the functions of $\mathbb{C}^{1 / 2}$ and $\mathbb{C}^{-1 / 2}$, respectively. This appendix is, therefore, devoted towards providing the $\mathbb{C}$ derivatives of $I_{4}^{e r}$ and $I_{5}^{e r}$.

In this context Hoger and Carlson (1984) provide explicit expressions for $\mathrm{d} \mathbb{C}^{1 / 2} / \mathrm{d} \mathbb{C}$, which is derived from the solution of the tensor equation having a general form $\mathbb{A}+\mathbb{X} \mathbb{A}=\mathbb{Q}$. Thus, the derivative of $I_{4}^{\text {er }}$ with respect to $\mathbb{C}$ is computed via first considering the scalar equation

$$
\mathbb{C}^{1 / 2} \mathbb{C}^{1 / 2}: \operatorname{sym}\left(\mathbf{H} \otimes \mathcal{H}^{r}\right)=\mathbb{C}: \operatorname{sym}\left(\mathbf{H} \otimes \mathcal{H}^{r}\right) .
$$

Differentiating both sides of the last with respect to $\mathbb{C}$ yields

$$
\mathbb{C}^{1 / 2} \frac{\partial}{\partial \mathbb{C}}\left[\mathbb{C}^{1 / 2}: \operatorname{sym}\left(\mathbf{H} \otimes \mathcal{H}^{r}\right)\right]+\frac{\partial}{\partial \mathbb{C}}\left[\mathbb{C}^{1 / 2}: \operatorname{sym}\left(\mathbf{H} \otimes \mathcal{H}^{r}\right)\right] \mathbb{C}^{1 / 2}=\operatorname{sym}\left(\mathbf{H} \otimes \mathcal{H}^{r}\right),
$$

which can be rephrased such that

$$
\mathbb{C}^{1 / 2} \frac{\partial I_{4}^{e r}}{\partial \mathbb{C}}+\frac{\partial I_{4}^{e r}}{\partial \mathbb{C}} \mathbb{C}^{1 / 2}=\operatorname{sym}\left(\mathbf{H} \otimes \mathcal{H}^{r}\right)
$$

having the same general form of $\mathbb{A} \mathbb{X}+\mathbb{X} \mathbb{A}=\mathbb{Q}$. The solution to (B.3) is thus given by (Hoger and Carlson, 1984)

$$
\begin{aligned}
\frac{\partial I_{4}^{e r}}{\partial \mathbb{C}}= & \frac{4}{\Delta}\left[I_{1}^{\mathrm{U}} \mathbb{C} \operatorname{sym}\left(\mathbf{H} \otimes \mathcal{H}^{r}\right) \mathbb{C}-\left(I_{1}^{\mathrm{U}}\right)^{2}\left\{\mathbb{C} \operatorname{sym}\left(\mathbf{H} \otimes \mathcal{H}^{r}\right) \mathbb{C}^{1 / 2}+\mathbb{C}^{1 / 2} \operatorname{sym}\left(\mathbf{H} \otimes \mathcal{H}^{r}\right) \mathbb{C}\right\}+\right. \\
& \left(I_{1}^{\mathrm{U}} I_{2}^{\mathrm{U}}-I_{3}^{\mathrm{U}}\right)\left\{\mathbb{C} \operatorname{sym}\left(\mathbf{H} \otimes \mathcal{H}^{r}\right)+\operatorname{sym}\left(\mathbf{H} \otimes \mathcal{H}^{r}\right) \mathbb{C}\right\}+\left(\left(I_{1}^{\mathrm{U}}\right)^{3}+I_{3}^{\mathrm{U}}\right) \mathbb{C}^{1 / 2} \operatorname{sym}\left(\mathbf{H} \otimes \mathcal{H}^{r}\right) \mathbb{C}^{1 / 2}- \\
& \left.\left(I_{1}^{\mathrm{U}}\right)^{2} I_{2}^{\mathrm{U}}\left\{\mathbb{C}^{1 / 2} \operatorname{sym}\left(\mathbf{H} \otimes \mathcal{H}^{r}\right)+\operatorname{sym}\left(\mathbf{H} \otimes \mathcal{H}^{r}\right) \mathbb{C}^{1 / 2}\right\}+\left\{\left(I_{1}^{\mathrm{U}}\right)^{2} I_{3}^{\mathrm{U}}+\left(I_{1}^{\mathrm{U}} I_{2}^{\mathrm{U}}-I_{3}^{\mathrm{U}}\right) I_{2}^{\mathrm{U}}\right\} \operatorname{sym}\left(\mathbf{H} \otimes \mathcal{H}^{r}\right)\right],
\end{aligned}
$$

where $I_{1}^{\mathrm{U}}, I_{2}^{\mathrm{U}}$ and $I_{3}^{\mathrm{U}}$ are three principal invariants of $\mathbf{U}=\mathbb{C}^{1 / 2}$ and $\Delta=8\left(I_{1}^{\mathrm{U}} I_{2}^{\mathrm{U}}-I_{3}^{\mathrm{U}}\right) I_{3}^{\mathrm{U}}$. Notice that $\partial I_{4}^{\text {er }} / \partial \mathbb{C}$ is symmetric.

Similarly, the derivative $\partial I_{5}^{e r} / \partial \mathbb{C}$ is computed via considering the scalar equation

$$
\mathbb{C}^{-1 / 2} \mathbb{C}^{-1 / 2}: \operatorname{sym}\left(\mathbf{H} \otimes \mathcal{H}^{r}\right)=\mathbb{C}^{-1}: \operatorname{sym}\left(\mathbf{H} \otimes \mathcal{H}^{r}\right)
$$

Differentiating both sides of the last with respect to $\mathbb{C}$ yields

$$
\mathbb{C}^{-1 / 2} \frac{\partial}{\partial \mathbb{C}}\left[\mathbb{C}^{-1 / 2}: \operatorname{sym}\left(\mathbf{H} \otimes \mathcal{H}^{r}\right)\right]+\frac{\partial}{\partial \mathbb{C}}\left[\mathbb{C}^{-1 / 2}: \operatorname{sym}\left(\mathbf{H} \otimes \mathcal{H}^{r}\right)\right] \mathbb{C}^{-1 / 2}=-\mathbb{C}^{-1} \operatorname{sym}\left(\mathbf{H} \otimes \mathcal{H}^{r}\right) \mathbb{C}^{-1}
$$

which can be rephrased such that

$$
\mathbb{C}^{1 / 2} \frac{\partial I_{5}^{e r}}{\partial \mathbb{C}}+\frac{\partial I_{5}^{e r}}{\partial \mathbb{C}} \mathbb{C}^{1 / 2}=-\mathbb{C}^{-1 / 2} \operatorname{sym}\left(\mathbf{H} \otimes \mathcal{H}^{r}\right) \mathbb{C}^{-1 / 2}
$$


having the same general form of $\mathbb{A} \mathbb{X}+\mathbb{X} \mathbb{A}=\mathbb{Q}$. The solution to (B.7) is thus given by (Hoger and Carlson, 1984)

$$
\begin{aligned}
& \frac{\partial I_{5}^{e r}}{\partial \mathbb{C}}=-\frac{4}{\Delta}\left[I_{1}^{\mathrm{U}} \mathbb{C}^{1 / 2} \operatorname{sym}\left(\mathbf{H} \otimes \mathcal{H}^{r}\right) \mathbb{C}^{1 / 2}-\left(I_{1}^{\mathrm{U}}\right)^{2}\left\{\mathbb{C}^{1 / 2} \operatorname{sym}\left(\mathbf{H} \otimes \mathcal{H}^{r}\right)+\operatorname{sym}\left(\mathbf{H} \otimes \mathcal{H}^{r}\right) \mathbb{C}^{1 / 2}\right\}+\right. \\
& \left(I_{1}^{\mathrm{U}} I_{2}^{\mathrm{U}}-I_{3}^{\mathrm{U}}\right)\left\{\mathbb{C}^{1 / 2} \operatorname{sym}\left(\mathbf{H} \otimes \mathcal{H}^{r}\right) \mathbb{C}^{-1 / 2}+\mathbb{C}^{-1 / 2} \operatorname{sym}\left(\mathbf{H} \otimes \mathcal{H}^{r}\right) \mathbb{C}^{1 / 2}\right\}+\left(\left(I_{1}^{\mathrm{U}}\right)^{3}+I_{3}^{\mathrm{U}}\right) \operatorname{sym}\left(\mathbf{H} \otimes \mathcal{H}^{r}\right)- \\
& \left.\left(I_{1}^{\mathrm{U}}\right)^{2} I_{2}^{\mathrm{U}}\left\{\operatorname{sym}\left(\mathbf{H} \otimes \mathcal{H}^{r}\right) \mathbb{C}^{-1 / 2}+\mathbb{C}^{-1 / 2} \operatorname{sym}\left(\mathbf{H} \otimes \mathcal{H}^{r}\right)\right\}+\left\{\left(I_{1}^{\mathrm{U}}\right)^{2} I_{3}^{\mathrm{U}}+\left(I_{1}^{\mathrm{U}} I_{2}^{\mathrm{U}}-I_{3}^{\mathrm{U}}\right) I_{2}^{\mathrm{U}}\right\} \mathbb{C}^{-1 / 2} \operatorname{sym}\left(\mathbf{H} \otimes \mathcal{H}^{r}\right) \mathbb{C}^{-1 / 2}\right] .
\end{aligned}
$$

Notice that $\partial I_{5}^{e r} / \partial \mathbb{C}$ is also symmetric.

\section{References}

Alapan, Y., Karacakol, A.C., Guzelhan, S.N., Isik, I., Sitti, M., 2020. Reprogrammable shape morphing of magnetic soft machines. Science Advances 6, eabc6414. URL: https://doi.org/10.1126/sciadv.abc6414, doi:10.1126/sciadv.abc6414.

Anoukou, K., Brenner, R., Hong, F., Pellerin, M., Danas, K., 2018. Random distribution of polydisperse ellipsoidal inclusions and homogenization estimates for porous elastic materials. Comp. Struct. 210, $87-101$. URL: http://www.sciencedirect.com/science/article/ pii/S0045794918304565, doi:https://doi.org/10.1016/j.compstruc.2018.08.006.

Bennett, K., Regueiro, R., Borja, R., 2016. Finite strain elastoplasticity considering the eshelby stress for materials undergoing plastic volume change. International Journal of Plasticity 77, 214-245. URL: https://doi.org/10.1016/j.ijplas.2015.10.007, doi:10.1016/j.ijplas . 2015.10.007.

Bodelot, L., Voropaieff, J.P., Pössinger, T., 2017. Experimental investigation of the coupled magneto-mechanical response in magnetorheological elastomers. Experimental Mechanics 58, 207-221. URL: https://doi.org/10.1007/s11340-017-0334-7, doi:10.1007/ s11340-017-0334-7.

Brown, W.F., 1963. Micromagnetics. 18, interscience publishers.

Brown, W.F., 1966. Magnetoelastic interactions. volume 9. Springer.

Chen, Y.C., Wheeler, L., 1993. Derivatives of the stretch and rotation tensors. Journal of Elasticity 32, 175-182. URL: https://doi.org/ 10.1007/bf00131659, doi:10.1007/bf00131659.

Choi, K.M., Rogers, J.A., 2003. A photocurable poly(dimethylsiloxane) chemistry designed for soft lithographic molding and printing in the nanometer regime. Journal of the American Chemical Society 125, 4060-4061. URL: https://doi.org/10.1021/ja029973k, doi:10.1021/ ja029973k.

Danas, K., 2017. Effective response of classical, auxetic and chiral magnetoelastic materials by use of a new variational principle. Journal of the Mechanics and Physics of Solids 105, 25-53. URL: https://doi.org/10.1016/j.jmps.2017.04.016, doi:10.1016/j.jmps.2017.04.016.

Danas, K., Kankanala, S., Triantafyllidis, N., 2012. Experiments and modeling of iron-particle-filled magnetorheological elastomers. Journal of the Mechanics and Physics of Solids 60, 120 - 138. URL: http://www.sciencedirect.com/science/article/pii/S0022509611001736, doi:10.1016/j.jmps.2011.09.006.

Danas, K., Ponte Castañeda, P., 2009. A finite-strain model for anisotropic viscoplastic porous media: I - theory. European Journal of Mechanics - A/Solids 28, 387 - 401. URL: http://www.sciencedirect.com/science/article/pii/S0997753808001150, doi:https://doi. $\mathrm{org} / 10.1016 / \mathrm{j}$. euromechsol.2008.11.002.

Dashner, P.A., 1993. An objective kinematical formalism for the modeling of elastic-plastic materials subject to large deformation. International Journal of Solids and Structures 30, 2661-2672. URL: https://doi.org/10.1016/0020-7683(93) 90104-f, doi:10.1016/0020-7683(93) 90104-f.

Deng, X., Liu, Z., Yu, H., Xiao, Z., Zhang, G., 2015. Isotropic and anisotropic nanocrystalline NdFeB bulk magnets prepared by binder-free high-velocity compaction technique. Journal of Magnetism and Magnetic Materials 390, 26-30. URL: https://doi.org/10.1016/j.jmmm. 2015.04.075, doi:10.1016/j.jmmm.2015.04.075.

DeSimone, A., James, R.D., 2002. A constrained theory of magnetoelasticity. Journal of the Mechanics and Physics of Solids 50, 283-320. URL: https://doi.org/10.1016/s0022-5096(01)00050-3, doi:10.1016/s0022-5096(01)00050-3.

Dorfmann, A., Ogden, R., 2003. Magnetoelastic modelling of elastomers. European Journal of Mechanics-A/Solids 22, 497-507. URL: https://doi.org/10.1016/s0997-7538(03)00067-6, doi:10.1016/s0997-7538(03)00067-6.

Dorfmann, A., Ogden, R., 2004. Nonlinear magnetoelastic deformations of elastomers. Acta Mechanica 167, 13-28. URL: https://doi.org/ 10.1007/s00707-003-0061-2, doi:10.1007/s00707-003-0061-2.

Galipeau, E., Ponte Castañeda, P., 2013. A finite-strain constitutive model for magnetorheological elastomers: Magnetic torques and fiber rotations. Journal of the Mechanics and Physics of Solids 61, 1065-1090. URL: https://doi.org/10.1016/j.jmps.2012.11.007, doi:10. $1016 / j \cdot j m p s .2012 .11 .007$.

Garcia-Gonzalez, D., Hossain, M., 2020. A microstructural-based approach to model magneto-viscoelastic materials at finite strains. International Journal of Solids and Structures URL: http://www.sciencedirect.com/science/article/pii/S0020768320304224, doi:https: //doi.org/10.1016/j.ijsolstr.2020.10.028.

Garcia-Gonzalez, D., Landis, C.M., 2020. Magneto-diffusion-viscohyperelasticity for magneto-active hydrogels: Rate dependences across time scales. Journal of the Mechanics and Physics of Solids 139, 103934. URL: http://www.sciencedirect.com/science/article/pii/ S0022509620301708, doi:https://doi.org/10.1016/j.jmps.2020.103934.

Garrell, M.G., Ma, B.M., Shih, A.J., Lara-Curzio, E., Scattergood, R.O., 2003. Mechanical properties of polyphenylene-sulfide (PPS) bonded nd-fe-b permanent magnets. Materials Science and Engineering: A 359, 375-383. URL: https://doi.org/10.1016/s0921-5093(03) 00400-3, doi:10.1016/s0921-5093(03)00400-3.

Ginder, J.M., Nichols, M.E., Elie, L.D., Clark, S.M., 2000. Controllable-stiffness components based on magnetorheological elastomers, in: Wereley, N.M. (Ed.), Smart Structures and Materials 2000: Smart Structures and Integrated Systems, SPIE. URL: https://doi.org/10. $1117 / 12.388844$, doi:10.1117/12.388844.

Ginder, J.M., Nichols, M.E., Elie, L.D., Tardiff, J.L., 1999. Magnetorheological elastomers: properties and applications, in: Smart Structures and Materials 1999: Smart Materials Technologies, International Society for Optics and Photonics. pp. 131-139. URL: https://doi.org/ 10.1117/12.352787, doi:10.1117/12.352787.

Green, A.E., Naghdi, P.M., 1965. A general theory of an elastic-plastic continuum. Archive for Rational Mechanics and Analysis 18, 251-281. URL: https://doi.org/10.1007/bf00251666, doi:10.1007/bf00251666.

Gurtin, M.E., 1982. An introduction to continuum mechanics. Academic press.

Hackl, K., Fischer, F.D., 2007. On the relation between the principle of maximum dissipation and inelastic evolution given by dissipation potentials. Proceedings of the Royal Society A: Mathematical, Physical and Engineering Sciences 464, 117-132. URL: https://doi.org/ 10.1098/rspa.2007.0086, doi:10.1098/rspa.2007.0086. 
Halphen, B., Son Nguyen, Q., 1975. Sur les matériaux standard généralisés. Journal de Mécanique 14, 39-63.

Hilber, W., Jakoby, B., 2012. Controlled liquid flow in a microfluidic network with pressure sensitive valves based on polydimethylsiloxane (PDMS)/neodymium (NdFeB) composites. Procedia Engineering 47, 382-385. URL: https://doi.org/10.1016/j.proeng.2012.09.163, doi:10.1016/j.proeng.2012.09.163.

Hoger, A., Carlson, D.E., 1984. On the derivative of the square root of a tensor and Guo's rate theorems. Journal of Elasticity 14, 329-336. URL: https://doi.org/10.1007/bf00041141, doi:10.1007/bf00041141.

Hossain, M., Saxena, P., Steinmann, P., 2015. Modelling the curing process in magneto-sensitive polymers: Rate-dependence and shrinkage. International Journal of Non-Linear Mechanics 74, 108 - 121. URL: http://www.sciencedirect.com/science/article/pii/ S0020746215000797, doi:https://doi.org/10.1016/j.ijnonlinmec.2015.04.008.

Huber, C., Abert, C., Bruckner, F., Groenefeld, M., Schuschnigg, S., Teliban, I., Vogler, C., Wautischer, G., Windl, R., Suess, D., 2017. 3d printing of polymer-bonded rare-earth magnets with a variable magnetic compound fraction for a predefined stray field. Scientific Reports 7. URL: https://doi.org/10.1038/s41598-017-09864-0, doi:10.1038/s41598-017-09864-0.

Huber, C., Sepehri-Amin, H., Goertler, M., Groenefeld, M., Teliban, I., Hono, K., Suess, D., 2019. Coercivity enhancement of selective laser sintered NdFeB magnets by grain boundary infiltration. Acta Materialia 172, 66-71. URL: https://doi.org/10.1016/j.actamat.2019. 04.037, doi:10.1016/j.actamat.2019.04.037.

Huber, J.E., Fleck, N.A., 2001. Multi-axial electrical switching of a ferroelectric: theory versus experiment. Journal of the Mechanics and Physics of Solids 49, 785-811. URL: https://doi.org/10.1016/s0022-5096(00)00052-1, doi:10.1016/s0022-5096(00)00052-1.

Huber, J.E., Fleck, N.A., Landis, C.M., McMeeking, R.M., 1999. A constitutive model for ferroelectric polycrystals. Journal of the Mechanics and Physics of Solids 47, 1663-1697. URL: https://doi.org/10.1016/s0022-5096(98)00122-7, doi:10.1016/s0022-5096(98)00122-7.

Hutter, K., van de Ven, A., 1978. Field matter interactions in thermoelastic solids. lectures notes in physics vol. 88.

James, R., Kinderlehrer, D., 1993. Theory of magnetostriction with applications to tbxdy1-xfe2. Phil. Mag. B 68, 237-274.

Johnston, I.D., McCluskey, D.K., Tan, C.K.L., Tracey, M.C., 2014. Mechanical characterization of bulk sylgard 184 for microfluidics and microengineering. Journal of Micromechanics and Microengineering 24, 035017. URL: https://doi.org/10.1088/0960-1317/24/3/035017, doi:10.1088/0960-1317/24/3/035017.

Jolly, M.R., Carlson, J.D., Muñoz, B.C., Bullions, T.A., 1996. The magnetoviscoelastic response of elastomer composites consisting of ferrous particles embedded in a polymer matrix. Journal of Intelligent Material Systems and Structures 7, 613-622. URL: https://doi.org/10. $1177 / 1045389 \times 9600700601$, doi:10.1177/1045389x9600700601.

Kaidarova, A., Khan, M.A., Amara, S., Geraldi, N.R., Karimi, M.A., Shamim, A., Wilson, R.P., Duarte, C.M., Kosel, J., 2018. Tunable, flexible composite magnets for marine monitoring applications. Advanced Engineering Materials 20, 1800229. URL: https://doi.org/10. 1002/adem. 201800229, doi:10.1002/adem. 201800229.

Kalina, K.A., Brummund, J., Metsch, P., Kästner, M., Borin, D.Y., Linke, J.M., Odenbach, S., 2017. Modeling of magnetic hystereses in soft mres filled with ndfeb particles. Smart Materials and Structures 26, 105019. URL: https://doi.org/10.1088/1361-665x/aa7f81, doi:10.1088/1361-665x/aa7f81.

Kalina, K.A., Metsch, P., Brummund, J., Kästner, M., 2020. A macroscopic model for magnetorheological elastomers based on microscopic simulations. International Journal of Solids and Structures 193-194, 200-212. URL: https://doi.org/10.1016/j.ijsolstr.2020.02.028, doi:10.1016/j.ijsolstr.2020.02.028.

Kalina, K.A., Metsch, P., Kästner, M., 2016. Microscale modeling and simulation of magnetorheological elastomers at finite strains: A study on the influence of mechanical preloads. International Journal of Solids and Structures 102-103, 286-296. URL: https://doi.org/10.1016/ j.ijsolstr.2016.10.019, doi:10.1016/j.ijsolstr.2016.10.019.

Kankanala, S., Triantafyllidis, N., 2004. On finitely strained magnetorheological elastomers. Journal of the Mechanics and Physics of Solids 52, 2869-2908. URL: https://doi.org/10.1016/j.jmps.2004.04.007, doi:10.1016/j.jmps.2004.04.007.

Keip, M.A., Rambausek, M., 2016. A multiscale approach to the computational characterization of magnetorheological elastomers. International Journal for Numerical Methods in Engineering 107, 338-360. URL: https://doi.org/10.1002/nme.5178, doi:10.1002/nme.5178.

Keip, M.A., Rambausek, M., 2017. Computational and analytical investigations of shape effects in the experimental characterization of magnetorheological elastomers. International Journal of Solids and Structures 121, 1-20. URL: https://doi.org/10.1016/j.ijsolstr. 2017.04.012, doi:10.1016/j.ijsolstr.2017.04.012.

Keip, M.A., Sridhar, A., 2018. A variationally consistent phase-field approach for micro-magnetic domain evolution at finite deformations. Journal of the Mechanics and Physics of Solids URL: https://doi.org/10.1016/j.jmps.2018.11.012, doi:10.1016/j.jmps.2018.11.012.

Kim, Y., Yuk, H., Zhao, R., Chester, S.A., Zhao, X., 2018. Printing ferromagnetic domains for untethered fast-transforming soft materials. Nature 558, 274-279. URL: https://doi.org/10.1038/s41586-018-0185-0, doi:10.1038/s41586-018-0185-0.

Klinkel, S., 2006. A phenomenological constitutive model for ferroelastic and ferroelectric hysteresis effects in ferroelectric ceramics. International Journal of Solids and Structures 43, 7197-7222. URL: https://doi.org/10.1016/j.ijsolstr.2006.03.008, doi:10.1016/j. ijsolstr.2006.03.008.

Kovetz, A., 2000. Electromagnetic theory. volume 975. Oxford University Press Oxford.

Kumar, A., Lopez-Pamies, O., 2016. On the two-potential constitutive modeling of rubber viscoelastic materials. Comptes Rendus Mécanique 344, 102-112. URL: https://doi.org/10.1016/j.crme.2015.11.004, doi:10.1016/j.crme.2015.11.004.

Landis, C.M., 2002. Fully coupled, multi-axial, symmetric constitutive laws for polycrystalline ferroelectric ceramics. Journal of the Mechanics and Physics of Solids 50, 127-152. URL: https://doi.org/10.1016/s0022-5096(01)00021-7, doi:10.1016/s0022-5096(01)00021-7.

Lefèvre, V., Danas, K., Lopez-Pamies, O., 2017. A general result for the magnetoelastic response of isotropic suspensions of iron and ferrofluid particles in rubber, with applications to spherical and cylindrical specimens. Journal of the Mechanics and Physics of Solids $107,343-364$. URL: https://doi.org/10.1016/j.jmps.2017.06.017, doi:10.1016/j.jmps.2017.06.017.

Lefèvre, V., Danas, K., Lopez-Pamies, O., 2019. Two families of explicit models constructed from a homogenization solution for the magnetoelastic response of mres containing iron and ferrofluid particles. International Journal of Non-Linear Mechanics URL: http://www.sciencedirect.com/science/article/pii/S0020746219306237, doi:https://doi.org/10.1016/j.ijnonlinmec.2019.103362.

Lefèvre, V., Danas, K., Lopez-Pamies, O., 2020. Two families of explicit models constructed from a homogenization solution for the magnetoelastic response of MREs containing iron and ferrofluid particles. International Journal of Non-Linear Mechanics 119, 103362. URL: http://www.sciencedirect.com/science/article/pii/S0020746219306237, doi:10.1016/j.ijnonlinmec.2019.103362.

Linke, J.M., Borin, D.Y., Odenbach, S., 2016. First-order reversal curve analysis of magnetoactive elastomers. RSC Advances 6, $100407-100416$. URL: https://doi.org/10.1039/c6ra23435f, doi:10.1039/c6ra23435f.

Linnemann, K., Klinkel, S., Wagner, W., 2009. A constitutive model for magnetostrictive and piezoelectric materials. International Journal of Solids and Structures 46, 1149-1166. URL: https://doi.org/10.1016/j.ijsolstr.2008.10.014, doi:10.1016/j.ijsolstr.2008.10.014.

Livingston, J.D., 1981. A review of coercivity mechanisms (invited). Journal of Applied Physics 52, 2544-2548. URL: https://doi.org/10. 1063/1.328996, doi:10.1063/1.328996.

Lokander, M., Stenberg, B., 2003. Performance of isotropic magnetorheological rubber materials. Polymer Testing 22, 245-251. URL: https://doi.org/10.1016/s0142-9418(02)00043-0, doi:10.1016/s0142-9418(02)00043-0.

Lopez-Pamies, O., Goudarzi, T., Danas, K., 2013. The nonlinear elastic response of suspensions of rigid inclusions in rubber: II-a simple 
explicit approximation for finite-concentration suspensions. Journal of the Mechanics and Physics of Solids 61, 19-37. URL: https: //doi.org/10.1016/j.jmps.2012.08.013, doi:10.1016/j.jmps.2012.08.013.

McMeeking, R.M., Landis, C.M., 2002. A phenomenological multi-axial constitutive law for switching in polycrystalline ferroelectric ceramics. International Journal of Engineering Science 40, 1553-1577. URL: https://doi.org/10.1016/s0020-7225(02)00033-2, doi:10.1016/s0020-7225(02) 00033-2.

McMeeking, R.M., Landis, C.M., 2005. Electrostatic forces and stored energy for deformable dielectric materials. Journal of Applied Mechanics 72, 581-590. URL: https://doi.org/10.1115/1.1940661, doi:10.1115/1.1940661.

McMeeking, R.M., Landis, C.M., Jimenez, S.M., 2007. A principle of virtual work for combined electrostatic and mechanical loading of materials. International Journal of Non-Linear Mechanics 42, 831-838. URL: https://doi.org/10.1016/j.ijnonlinmec.2007.03.008, doi:10.1016/j.ijnonlinmec.2007.03.008.

Miehe, C., 2002. Strain-driven homogenization of inelastic microstructures and composites based on an incremental variational formulation. International Journal for Numerical Methods in Engineering 55, 1285-1322. URL: https://doi.org/10.1002/nme.515, doi:10.1002/nme. 515.

Miehe, C., Rosato, D., Kiefer, B., 2011. Variational principles in dissipative electro-magneto-mechanics: A framework for the macro-modeling of functional materials. International Journal for Numerical Methods in Engineering 86, 1225-1276. URL: https://doi.org/10.1002/nme. 3127, doi:10.1002/nme.3127.

Miehe, C., Schotte, J., Lambrecht, M., 2002. Homogenization of inelastic solid materials at finite strains based on incremental minimization principles. application to the texture analysis of polycrystals. Journal of the Mechanics and Physics of Solids 50, 2123-2167. URL: https://doi.org/10.1016/s0022-5096(02)00016-9, doi:10.1016/s0022-5096(02)00016-9.

Mukherjee, D., 2020. Theoretical and numerical modeling of magnetorheological elastomers comprising magnetically soft and hard particles. $\mathrm{PhD}$ Thesis

Mukherjee, D., Bodelot, L., Danas, K., 2020. Microstructurally-guided explicit continuum models for isotropic magnetorheological elastomers with iron particles. International Journal of Non-Linear Mechanics, 103380URL: https://doi.org/10.1016/j.ijnonlinmec.2019.103380, doi:10.1016/j.ijnonlinmec.2019.103380.

Mukherjee, D., Danas, K., 2019. An evolving switching surface model for ferromagnetic hysteresis. Journal of Applied Physics 125, 033902. URL: https://doi.org/10.1063/1.5051483, doi:10.1063/1.5051483.

Mukherjee, D., Rambausek, M., Danas, K., 2021. Abaqus uel subroutine for hard and soft magnetorheological elastomers. doi:10.5281/ zenodo. 4588578

Ortiz, M., Stanier, L., 1999. The variational formulation of viscoplastic constitutive updates. Computer Methods in Applied Mechanics and Engineering 171, 419-444.

Pao, Y.H., Hutter, K., 1975. Electrodynamics for moving elastic solids and viscous fluids. Proceedings of the IEEE 63, 1011-1021. URL: https://doi.org/10.1109/proc.1975.9878, doi:10.1109/proc.1975.9878.

Park, S., Mondal, K., Treadway, R.M., Kumar, V., Ma, S., Holbery, J.D., Dickey, M.D., 2018. Silicones for stretchable and durable soft devices: Beyond sylgard-184. ACS Applied Materials \& Interfaces 10, 11261-11268. URL: https://doi.org/10.1021/acsami.7b18394, doi:10.1021/acsami.7b18394.

Pelteret, J.P., Davydov, D., McBride, A., Vu, D.K., Steinmann, P., 2016. Computational electro-elasticity and magneto-elasticity for quasiincompressible media immersed in free space. International Journal for Numerical Methods in Engineering 108, 1307-1342. URL: http: //onlinelibrary.wiley.com/doi/10.1002/nme.5254/abstract, doi:10.1002/nme.5254.

Ponte Castañeda, P., Galipeau, E., 2011. Homogenization-based constitutive models for magnetorheological elastomers at finite strain. Journal of the Mechanics and Physics of Solids 59, 194-215. URL: https://doi.org/10.1016/j.jmps.2010.11.004, doi:10.1016/j.jmps.2010.11. 004.

Psarra, E., Bodelot, L., Danas, K., 2017. Two-field surface pattern control via marginally stable magnetorheological elastomers. Soft Matter 13, 6576-6584. URL: https://doi.org/10.1039/c7sm00996h, doi:10.1039/c7sm00996h.

Psarra, E., Bodelot, L., Danas, K., 2019. Wrinkling to crinkling transitions and curvature localization in a magnetoelastic film bonded to a non-magnetic substrate. Journal of the Mechanics and Physics of Solids 133, 103734. URL: https://doi.org/10.1016/j.jmps .2019.103734, doi:10.1016/j.jmps.2019.103734.

Ren, Z., Hu, W., Dong, X., Sitti, M., 2019. Multi-functional soft-bodied jellyfish-like swimming. Nature Communications $10 . \quad$ URL: https://doi.org/10.1038/s41467-019-10549-7, doi:10.1038/s41467-019-10549-7.

Rigbi, Z., Jilkén, L., 1983. The response of an elastomer filled with soft ferrite to mechanical and magnetic influences. Journal of Magnetism and Magnetic Materials 37, 267-276. URL: https://doi.org/10.1016/0304-8853(83) 90055-0, doi:10.1016/0304-8853(83)90055-0.

Robinson, F., 1975. Electromagnetic stress and momentum in matter. Physics Reports 16, 313-354. URL: https://doi.org/10.1016/ 0370-1573(75) 90057-5, doi:10.1016/0370-1573(75)90057-5.

Rosato, D., Miehe, C., 2014. Dissipative ferroelectricity at finite strains. variational principles, constitutive assumptions and algorithms. International Journal of Engineering Science 74, 162-189. URL: https://doi.org/10.1016/j.ijengsci.2013.08.007, doi:10.1016/j. ijengsci.2013.08.007.

Royet, D., Hériveaux, Y., Marchalot, J., Scorretti, R., Dias, A., Dempsey, N.M., Bonfim, M., Simonet, P., Frénéa-Robin, M., 2017. Using injection molding and reversible bonding for easy fabrication of magnetic cell trapping and sorting devices. Journal of Magnetism and Magnetic Materials 427, 306-313. URL: https://doi.org/10.1016/j.jmmm.2016.10.102, doi:10.1016/j.jmmm.2016.10.102.

Sánchez, P.A., Gundermann, T., Dobroserdova, A., Kantorovich, S.S., Odenbach, S., 2018. Importance of matrix inelastic deformations in the initial response of magnetic elastomers. Soft Matter URL: https://doi.org/10.1039/c7sm02366a, doi:10.1039/c7sm02366a.

Saxena, P., Hossain, M., Steinmann, P., 2013. A theory of finite deformation magneto-viscoelasticity. International Journal of Solids and Structures 50, 3886 - 3897. URL: http://www.sciencedirect.com/science/article/pii/S0020768313003041, doi:https://doi.org/10. 1016/j.ijsolstr.2013.07.024.

Saxena, P., Hossain, M., Steinmann, P., 2014. Nonlinear magneto-viscoelasticity of transversally isotropic magneto-active polymers. Proceedings of the Royal Society A: Mathematical, Physical and Engineering Sciences 470, 20140082. URL: https://doi.org/10.1098\%2Frspa. 2014.0082, doi:10.1098/rspa.2014.0082.

Schöberl, J., 1997. Netgen an advancing front 2d/3d-mesh generator based on abstract rules. J. Comput Visual Sci 1, 41-52.

Schümann, M., Odenbach, S., 2017. In-situ observation of the particle microstructure of magnetorheological elastomers in presence of mechanical strain and magnetic fields. Journal of Magnetism and Magnetic Materials 441, 88-92. URL: https://doi.org/10.1016/j. jmmm.2017.05.024, doi:10.1016/j.jmmm.2017.05.024.

Segurado, J., Llorca, J., 2002. A numerical approximation to the elastic properties of sphere-reinforced composites. Journal of the Mechanics and Physics of Solids 50, 2107-2121.

Sitti, M., Wiersma, D.S., 2020. Pros and cons: Magnetic versus optical microrobots. Advanced Materials , 1906766URL: https://doi.org/ 10.1002/adma.201906766, doi:10.1002/adma.201906766.

Suo, Z., Zhao, X., Greene, W., 2008. A nonlinear field theory of deformable dielectrics. Journal of the Mechanics and Physics of Solids 56, 467-486. URL: https://doi.org/10.1016/j.jmps.2007.05.021, doi:10.1016/j.jmps.2007.05.021. 
Tarantino, M., Zerhouni, O., Danas, K., 2019. Random 3D-printed isotropic composites with high volume fraction of pore-like polydisperse inclusions and near-optimal elastic stiffness. Acta Materialia 175, 331-340. URL: https://doi.org/10.1016/j.actamat.2019.06.020, doi:10.1016/j.actamat.2019.06.020.

Taylor, A.P., Cuervo, C.V., Arnold, D.P., Velasquez-Garcia, L.F., 2019. Fully 3d-printed, monolithic, mini magnetic actuators for lowcost, compact systems. Journal of Microelectromechanical Systems 28, 481-493. URL: https://doi.org/10.1109/jmems.2019.2910215, doi:10.1109/jmems.2019.2910215.

Tiersten, H.F., 1964. Coupled Magnetomechanical Equations for Magnetically Saturated Insulators. Journal of Mathematical Physics 5, 1298-1318. URL: https://aip.scitation.org/doi/abs/10.1063/1.1704239, doi:10.1063/1.1704239.

Tiersten, H.F., 1965. Variational Principle for Saturated Magnetoelastic Insulators. Journal of Mathematical Physics 6, 779-787. URL: http://aip.scitation.org/doi/abs/10.1063/1.1704334, doi:10.1063/1.1704334.

Wang, Z., Xiang, C., Yao, X., Floch, P.L., Mendez, J., Suo, Z., 2019. Stretchable materials of high toughness and low hysteresis. Proceedings of the National Academy of Sciences 116, 5967-5972. URL: https://doi.org/10.1073/pnas.1821420116, doi:10.1073/pnas.1821420116.

Wilkins, M., 1964. Calculation of elastic-plastic flow, in: Alder, B., Fernback, S. Rotenberg, M. (Eds.), Methods of Computational Physics. Academic Press, New York. volume 3, pp. 271-277.

Zerhouni, O., Tarantino, M., Danas, K., 2019. Numerically-aided 3D printed random isotropic porous materials approaching the hashinshtrikman bounds. Composites Part B: Engineering 156, 344-354. URL: https://doi.org/10.1016/j.compositesb.2018.08.032, doi:10. 1016/j.compositesb.2018.08.032.

Zhao, R., Kim, Y., Chester, S.A., Sharma, P., Zhao, X., 2019. Mechanics of hard-magnetic soft materials. Journal of the Mechanics and Physics of Solids 124, 244-263. URL: https://doi.org/10.1016/j.jmps.2018.10.008, doi:10.1016/j.jmps.2018.10.008.

Zhou, R., Surendran, A.N., Mejulu, M., Lin, Y., 2020. Rapid microfluidic mixer based on ferrofluid and integrated microscale NdFeB-PDMS magnet. Micromachines 11, 29. URL: https://doi.org/10.3390/mi11010029, doi:10.3390/mi11010029. 